PREPARED FOR THE U.S. DEPARTMENT OF ENERGY, UNDER CONTRACT DE-AC02-76CH03073

PPPL-3584

PPPL-3584

UC-70

Unusual Low-frequency Magnetic Perturbations in TFTR

H. Takahashi, E.D. Fredrickson, and M.S. Chance

June 2001

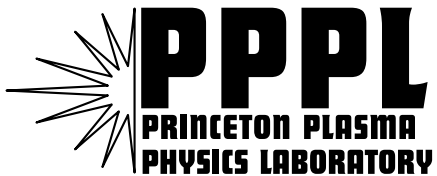

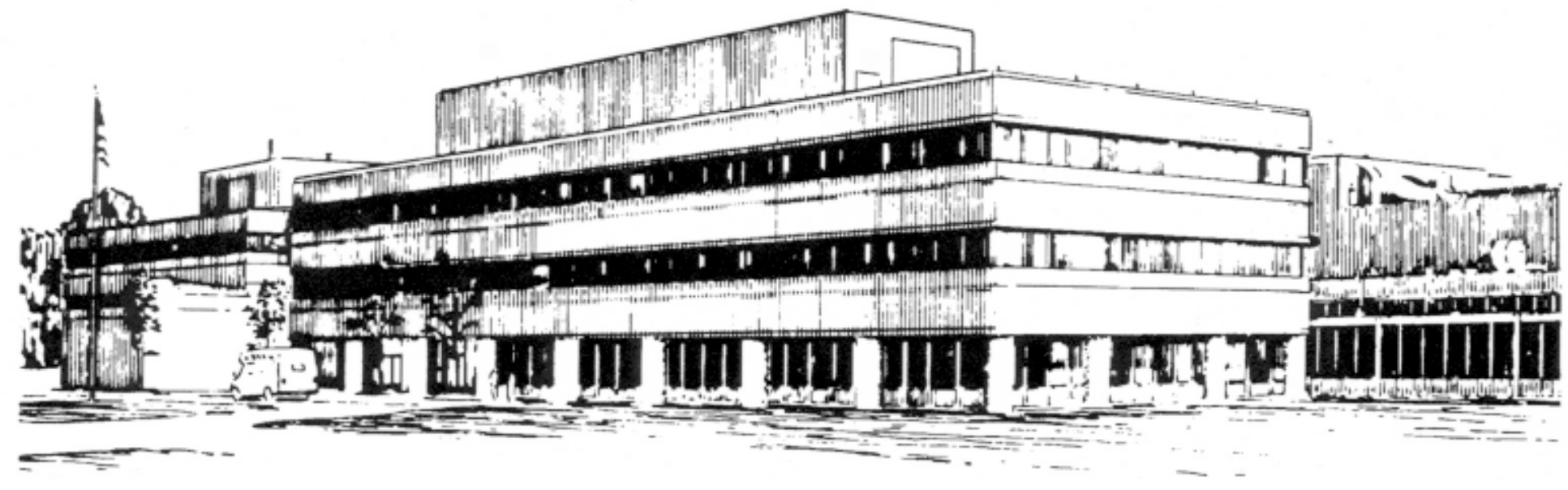

PRINCETON PLASMA PHYSICS LABORATORY PRINCETON UNIVERSITY, PRINCETON, NEW JERSEY 


\section{PPPL Reports Disclaimer}

This report was prepared as an account of work sponsored by an agency of the United States Government. Neither the United States Government nor any agency thereof, nor any of their employees, makes any warranty, express or implied, or assumes any legal liability or responsibility for the accuracy, completeness, or usefulness of any information, apparatus, product, or process disclosed, or represents that its use would not infringe privately owned rights. Reference herein to any specific commercial product, process, or service by trade name, trademark, manufacturer, or otherwise, does not necessarily constitute or imply its endorsement, recommendation, or favoring by the United States Government or any agency thereof. The views and opinions of authors expressed herein do not necessarily state or reflect those of the United States Government or any agency thereof.

\section{Availability}

This report is posted on the U.S. Department of Energy's Princeton Plasma Physics Laboratory Publications and Reports web site in Calendar Year 2001. The home page for PPPL Reports and Publications is: http://www.pppl.gov/pub_report/

DOE and DOE Contractors can obtain copies of this report from:

U.S. Department of Energy

Office of Scientific and Technical Information

DOE Technical Information Services (DTIS)

P.O. Box 62

Oak Ridge, TN 37831

Telephone: (865) 576-8401

Fax: (865) 576-5728

Email: reports@adonis.osti.gov

This report is available to the general public from:

National Technical Information Service

U.S. Department of Commerce

5285 Port Royal Road

Springfield, VA 22161

Telephone: 1-800-553-6847 or

(703) 605-6000

Fax: (703) 321-8547

Internet: http://www.ntis.gov/ordering.htm 


\title{
UNUSUAL LOW-FREQUENCY MAGNETIC PERTURBATIONS IN TFTR TOKAMAK
}

\author{
H. Takahashi, E.D. Fredrickson, and M.S. Chance \\ Princeton Plasma Physics Laboratory, Princeton University \\ Princeton, New Jersey 08543-0451
}

\begin{abstract}
Low frequency magnetic perturbations $(\leq 30 \mathrm{kHz})$ observed in the Tokamak Fusion Test Reactor (TFTR) tokamak do not always conform to expectations from Magneto-Hydro-Dynamic (MHD) modes. The discrepancy between observations and expectations arises from the existence of three classes of magnetic perturbations in TFTR: (1) 'Edge Originated Magnetic Perturbations' (EOMP's), (2) 'Kink-Like Modes' (KLM's), and (3) Tearing Modes (TM's). The EOMP class has unusual magnetic phenomenon including up/down asymmetry in poloidal intensity variation that MHD modes alone cannot generate. The contributions of MHD modes in plasma edge regions are too small to explain the magnitude of observed EOMP perturbations. At least two-thirds, possibly nearly all, of magnetic perturbations in a typical EOMP originate from sources other than MHD modes. An EOMP has a unity toroidal harmonic number and a poloidal harmonic number close to a discharge's edge q-value. It produces little temperature fluctuations, except possibly in edge regions. The KLM class produces temperature fluctuations, mostly confined within the $\mathrm{q}=1$ surface with an ideal-mode-like structure, but generates little external magnetic perturbations. The TM class conforms generally to expectations from MHD modes. We propose that current flowing in the Scrape-Off-Layer (SOL) plasma is a possible origin of EOMP's.
\end{abstract}

\section{Contents}

1 INTRODUCTION 4

2 EXPERIMENTAL ENVIRONMENT $\quad 6$

2.1 TFTR Tokamak . . . . . . . . . . . . . . 6

2.2 Principal Diagnostics . . . . . . . . . . . . . . . . . 6

2.3 Computational Tools . . . . . . . . . . . . . . . . 8

2.4 Discharge Conditions . . . . . . . . . . . . . . . . . . . 9

3 MAGNETIC PERTURBATIONS IN TFTR 9

3.1 Frequency Spectra . . . . . . . . . . . . . . . . . 9 
3.2 Gallery of Perturbation Patterns . . . . . . . . . . . . . . 10

3.2 .1 Modulus Variation . . . . . . . . . . . . . . . . . 11

3.2 .2 Argument Variation . . . . . . . . . . . . . . . . . . 12

3.3 Closer Examinations . . . . . . . . . . . . . . . . . . . 16

3.4 Constituent Harmonics . . . . . . . . . . . . . . . . . . . . . . . . . 16

3.4.1 Data Analysis Approach . . . . . . . . . . . . . . 16

3.4 .2 Interpretations of Individual Discharges $\ldots \ldots \ldots \ldots$

3.5 Section Summary . . . . . . . . . . . . . . . . 20

4 THREE CLASSES OF PERTURBATIONS 21

4.1 Oscillating Temperature Profiles _ . . . . . . . . . . . . . . . 22

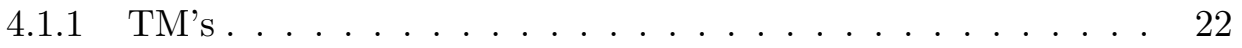

$4.1 .2 \quad$ KLM's . . . . . . . . . . . . . . . . . . . 24

4.1 .3 EOMP's ...................... 27

4.2 Phase Reversal . . . . . . . . . . . . . . . . . . . . . . . 27

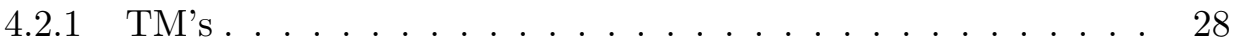

$4.2 .2 \quad$ KLM's . . . . . . . . . . . . . . . . . . . . . . 28

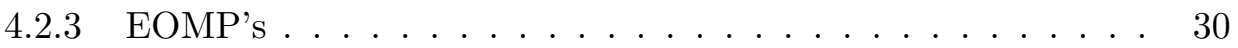

4.3 Buried Organized Activity . . . . . . . . . . . . . . . . 30

$4.3 .1 \quad$ TM's ........................... 31

$4.3 .2 \quad$ KLM's . . . . . . . . . . . . . . . . . . . . . . 32

$4.3 .3 \quad$ EOMP's . . . . . . . . . . . . . . . . . . . . 32

4.4 External Appearance . . . . . . . . . . . . . . . . . . . . 33

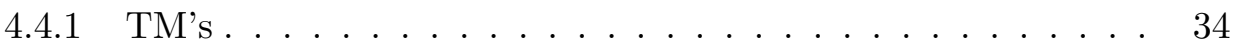

$4.4 .2 \quad$ KLM's . . . . . . . . . . . . . . . . . . . . . . . 35

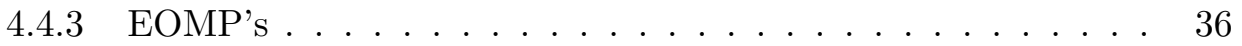

4.5 Spectral Time Evolution . . . . . . . . . . . . . . . . . 36

4.6 Spectral and Spatial Harmonics . . . . . . . . . . . . . . . . 38

4.7 Relationships among Classes . . . . . . . . . . . . . . . . 39

4.8 Summary of Characteristics of Three Classes . . . . . . . . . . . . . 40 
5 MHD-MODE CONTRIBUTIONS WITHIN EOMP

5.1 Magnetic Perturbations . . . . . . . . . . . . . . . . 42

5.1.1 Time and Frequency Domain Signals . . . . . . . . . . . . 42

5.1 .2 Poloidal Variations . . . . . . . . . . . . . . . . 43

5.2 Electron Temperature Fluctuations . . . . . . . . . . . . . . 46

5.2.1 Transforming Minor Radius . . . . . . . . . . . . . . . 46

5.2 .2 Constructing Temperature Profile . . . . . . . . . . . 47

5.2 .3 Temperature Fluctuations . . . . . . . . . . . . . . . . 48

5.2 .4 Noise Level . . . . . . . . . . . . . . . . . . . . . . . 50

5.2 .5 Flux Surface Displacement. . . . . . . . . . . . . 50

5.2.6 Flux Surface Motion and Temperature Fluctuations. . . . . . 51

5.2 .7 Hidden Islands . . . . . . . . . . . . . . . . . . . . . . 53

5.2 .8 Allowance for Errors . . . . . . . . . . . . . . . 55

5.2 .9 Summing Contributions . . . . . . . . . . . . 56

5.3 Section Summary . . . . . . . . . . . . . . . . 57

6 CONCLUSION $\quad 57$

$\begin{array}{lll}7 & \text { DISCUSSION } & 58\end{array}$

7.1 Implications for MHD Modes . . . . . . . . . . . . . . . 59

7.1 .1 Locked Mode Studies . . . . . . . . . . . . . . . . . . 59

7.1.2 Neoclassical Tearing Mode Studies . . . . . . . . . . . . . 60

7.1 .3 Resistive Wall Mode Studies . . . . . . . . . . . . . 60

7.2 Current in SOL Plasma . . . . . . . . . . . . . . 60

7.2 .1 Our Earlier Works . . . . . . . . . . . . . . . 61

7.2 .2 Our Present and Future Works . . . . . . . . . . . 62

7.2 .3 Historical Perspective . . . . . . . . . . . . . . . 62

8 ACKNOWLEGEMENT

A FIELD PATTERN IN VACUUM TOROIDAL GEOMETRY 64

A.1 Modifications to Simple Picture . . . . . . . . . . . . . . . . . 64 


\section{INTRODUCTION}

The tokamak has been at the forefront of plasma physics and nuclear fusion energy research for the last four decades. Spectacular advances in sophisticated diagnostics, coupled with a readily available massive computing power in recent years, have enabled probing of the plasma at an ever deeper and fundamental level of understanding. Yet, its future prospect as a reactor depends critically on whether or not it can sustain an Advanced Tokamak (AT) plasma regime[1-7] with high performance parameters for economical energy production.

Many tokamaks have produced an AT plasma for a brief period. But sustaining it has proven far more difficult. The demise of an AT plasma often occurs following the rise of low frequency magnetic phenomena. They include so-called Locked Modes (LM's)[8-18], Neoclassical Tearing Modes (NTM's)[19-24], Resistive Wall Modes (RWM's)[25-36], and Edge Localized Modes (ELM's)[37-39]. These phenomena have always been understood as a manifestation of a Magneto-HydroDynamic (MHD) instability, as inclusion of the term, 'mode,' in their names suggests. Success or failure in eliminating or controlling these magnetic phenomena may well be a key factor in deciding the tokamak's future as an energy producing reactor.

A large body of theoretical and experimental knowledge already exist on these low frequency magnetic phenomena. The intent of the present article is not to add to such 'MHD issues' as stability and growth of these modes. It examines rather experimental data from the Tokamak Fusion Test Reactor[40] (TFTR) that could influence views on the basic nature of some of these magnetic phenomena. It reports evidence that a part of large amplitude low frequency magnetic perturbations do not originate from MHD modes.

We will offer our thoughts on some potential consequences of the extraneous magnetic perturbations in a discussion section. But we state them here at the outset also, because they articulate our motivation and interest in the subject matter. Should the extraneous perturbations possess a component resonant on a singular surface in plasma interior regions, they could be significant to LM and NTM theories on a fundamental level. They could supply an additional destabilizing influence that these theories conventionally sought in structural error field and neoclassical current inside magnetic islands. Should the perturbations interact with both the tokamak's structures and MHD modes, they could act as an intermediary for momentum transfer - a mechanism of rotation slow down that LM theory traditionally found in the image current induced in tokamak structures. The perturbations would be an unwelcome intruder in a scheme to suppress RWM's by a feedback control that 
would not obey the feedback loop's intended causality relationship. The discovery could also lead to fundamentally different remedies for problems associated with sustaining an AT plasma that may be simpler and more economical than those conventionally suggested or attempted.

Figure 1 depicts a typical discharge situation addressed in this article. It shows the time evolution of the plasma current, NBI heating power, and magnetic perturbation field (Mirnov coil signal). The NBI heating power was on during part of the discharge. The perturbation field rose from a noise-like level in the pre-heating phase to a large amplitude during the heating period. Some adverse effects on the discharge occurred during the period of growing magnetic perturbations.

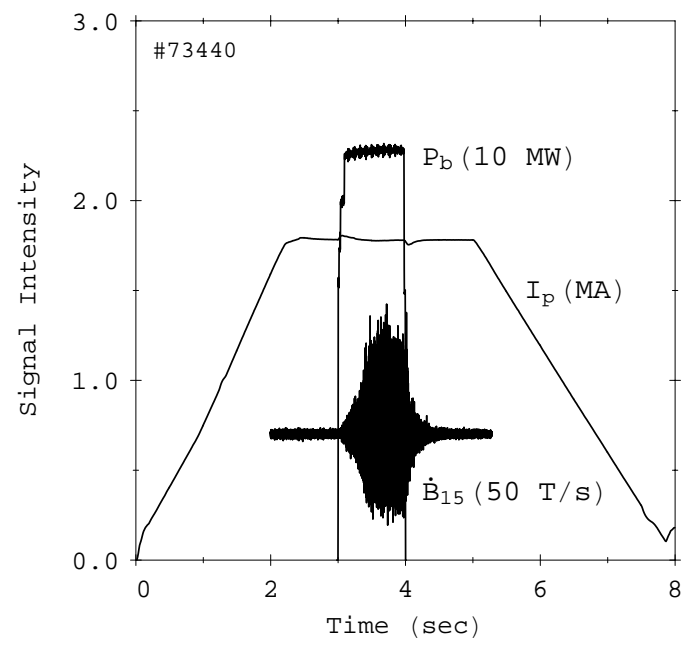

Fig. 1 A description of a typical situation studied in this article. $I_{p}$ is the plasma current, $P_{b}$ is the NBI heating power, and $\dot{B}_{15}$ is a signal from Mirnov coil \#15. The Mirnov signal has oscillations about zero at around $4.6 \mathrm{kHz}$. But the time scale used here cannot resolve their individual cycles, and the Mirnov trace appears as a darkened area. It is also shifted vertically into a range common to the other traces.

Similar scenarios are repeated countless times in TFTR and also quite likely in other tokamaks. It is thought in each such instance that heating rendered an MHD mode unstable, and its perturbation field generated Mirnov signals. This is a firmly entrenched interpretation, and leaves little room for any others. Yet, this article will demonstrate that this interpretation is in some instances largely incorrect, including this particular discharge and many others.

We are of course not suggesting that MHD modes do not exist in TFTR. We are instead proposing that something else exists that can get confused with MHD modes. When it appears alone, we have an opportunity to characterize it. We will seize those moments. When it appears together with MHD modes, as it often does, the picture becomes more complicated.

A model consisting of three classes of perturbations will be introduced to explain low frequency magnetic phenomena in TFTR: (1) 'Edge Originated Magnetic Perturbations' (EOMP's), (2) 'Kink-Like Modes' (KLM's), and (3) Tearing Modes (TM's). The first of these classes is an unusual magnetic perturbation. Only the last class has an established theoretical basis. EOMP's can have strong external magnetic perturbations and little internal electron temperature fluctuations. KLM's have internal temperature fluctuations akin to an ideal mode but little external magnetic perturbations. TM's generate magnetic islands as well as external magnetic 
perturbations.

We will assess an upper bound to contributions to EOMP's external magnetic perturbations from MHD modes that may have escaped detection because of noise and discrete measuring points of temperature diagnostics, and demonstrate that a major part of EOMP's magnetic perturbations originates from a source other than MHD modes.

\section{EXPERIMENTAL ENVIRONMENT}

\subsection{TFTR Tokamak}

The TFTR tokamak[40] produces a plasma with a circular poloidal cross-section. Its typical parameter ranges are: toroidal field (TF), $\left|B_{\phi}\right| \leq 5.9 \mathrm{~T}$ (at $R=2.52 \mathrm{~m}$ ), plasma current, $I_{p} \leq 3.0 \mathrm{MA}$, and plasma major and minor radii, $2.35 \leq R_{p} \leq$ $2.625 \mathrm{~m}$ and $0.70 \leq a_{p} \leq 0.975 \mathrm{~m}$, respectively.

A schematic poloidal cross section of the device is in Fig. 2. The vacuum vessel has a circular poloidal cross-section. Its major and minor radii are $2.65 \mathrm{~m}$ and $1.1 \mathrm{~m}$, respectively. The plasma is usually limited by toroidal 'bumper' limiters, nominally at the inboard midplane ('star' in the figure). They have the shape of a circular arc of a radius, $1.00 \mathrm{~m}$, in a poloidal cross-section, which subtends a poloidal angle, $\pm 60 \mathrm{deg}$ (measured from the inboard midplane), about the vacuum vessel center. The outboard side has poloidal limiters. These are meant for protecting Radio Frequency (RF) antennas, and are not usually used for defining the plasma aperture.

The plasma is heated primarily by Neutral Beam Injection (NBI), and sometimes by Ion Cyclotron Range of Frequency (ICRF) heating, either alone or in conjunction with NBI. The NBI heating system has 12 ion sources, 6 in a co-parallel direction and 6 in a counter-parallel direction with respect to the plasma current direction. Each ion source is capable of producing up to about 2.8 MW of power (in deuterium) and up to about 4 Newton-m of toroidal torque. Choosing various combinations of co- and counter-parallel ion sources alters the net momentum imparted to the plasma by neutral beams. 'Balanced' injection is often employed in which the net momentum is nearly zero.

\subsection{Principal Diagnostics}

Magnetic diagnostics, Electron Cyclotron Emission (ECE) diagnostics, Charge Exchange Recombination Spectroscopy (CHERS), Beam Emission Spectroscopy (BES), vacuum spectroscopy, Multi-channel Infra-Red Interferometer (MIRI), Motion Stark Effect (MSE) diagnostic, and an array of other standard tokamak diagnostics are available on TFTR. Measurements from many of these diagnostics are used for determining the state of the plasma. The present article is most directly 
concerned with the magnetic and ECE fluctuation diagnostics.

A main magnetic diagnostic[41] for detecting oscillatory perturbations is an array of magnetic coils ('Mirnov coils'). The ECE diagnostics[42,43] consist of two independent Grating Poly-chromators (GPC's) at two different toroidal locations, and an absolutely calibrated Michelson interferometer, all of which measure the electron temperature as a function of major radius and time. The GPC-1 instrument and Michelson interferometer share a common waveguide/horn. Locations of magnetic and ECE diagnostics are shown in a toroidal-poloidal angle plane in Fig. 3. The toroidal angle $(\phi)$ advances in a clock-wise direction when the tokamak is viewed from above. The poloidal angle $(\theta)$ is measured about the vacuum vessel center. It begins from the inboard midplane, and advances first in an upward direction.

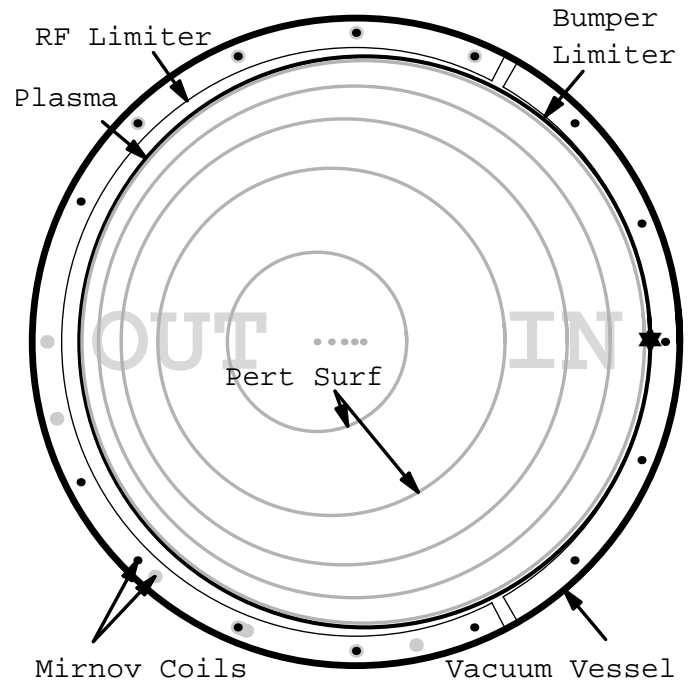

Fig. 2 Explains relationships among the vacuum vessel, limiters, Mirnov coils, and plasma. The tokamak symmetry axis is to the right of picture. Included also are a set of Shafranovshifted singular surfaces computed for an actual discharge.

The present investigation uses 30 Mirnov coils, all installed inside the vacuum vessel. They are on a Mirnov coil installation circle with a minor radius, $1.05 \mathrm{~m}$, whose center is at a major radius, $2.65 \mathrm{~m}$, and on the midplane. The axis of each coil is tangent to the circle. Fifteen of these coils are at the same toroidal angle, and are members of a poloidal array (PA) (Fig. 3). The remaining fifteen are at varying toroidal and poloidal locations, and are referred to as the non-poloidal array (NPA) coils. We will designate a coil's angular location by a pair of numbers in parentheses: e.g., Mirnov \#8(153/261) is coil \#8 at $\theta=153 \mathrm{deg}$ and $\phi=261 \mathrm{deg}$. Each coil has an effective coupling area of $\sim 550 \mathrm{~cm}^{2}$. Amplifiers used for all Mirnov signals are 'AC coupled' (equivalent to a high-pass filter with a $3 \mathrm{db}$ cut-off frequency of $\sim 10 \mathrm{~Hz}$ ). Digitizers record Mirnov signals at many different rates. The present investigation uses primarily 'fast' data sampled at $500 \mathrm{kHz}$ usually over eight inde- 
pendently selectable time windows, each $8.191 \mathrm{~ms}$ long ( $2^{12}$ time points), during a discharge.

Each GPC measures the intensity of second harmonic electron cyclotron emission at 20 discrete frequencies from which the relative electron temperature is determined. The total field ('mod B'), i.e., the toroidal field, including the diamagnetic effect, and the poloidal field is used in converting the frequencies to radial positions where the emission originates. The GPC measurement is taken usually over an approximate major radial range of $2.40-3.40 \mathrm{~m}$ at intervals of $5-6 \mathrm{~cm}$. Digitizers record GPC signals also at several different rates. The present investigation uses primarily 'fast' data sampled at $500 \mathrm{kHz}$ usually over eight independently selectable time windows, each $32.766 \mathrm{~ms}$ long ( $2^{14}$ time points), in a discharge. The absolute temperature scales of GPC's are usually calibrated at a single time point in the discharge against a temperature measurement based upon the Michelson interferometry.

The plasma extends over a range in which the magnetic field strength varies considerably. Overlapping of harmonic emissions from different regions of the plasma sometimes makes interpretation of GPC signals in inboard and outboard regions difficult. Non-thermal emission[43] from plasma interior region that becomes shifted down in frequency and detected by sensors intended for detecting signals emitted locally near outboard plasma edge also makes the measurement there difficult. We will use the term, 'GPC viewing domain,' for a range of plasma region covered by a GPC instrument.

\subsection{Computational Tools}

A number of computational analysis codes are available. The TRANSP code[44] is a one-dimensional time-dependent transport code, which has been in use in many research institutions around the world.

The SNAP code[45] is a one-dimensional time-independent transport code used extensively at the Princeton Plasma Physics Laboratory, both for a between-shots as well as off-line analyses.

The VACUUM code[46] solves the 3D Laplace's equation for the magnetic scalar potential in general azimuthally symmetric geometry in the region surrounding a plasma discharge which may or may not contain external conductors. In the context of the present article it can compute magnetic perturbations on the surface of conducting vacuum vessel walls that originate from a singular surface inside the plasma, under an assumption of a vacuum region between the two surfaces.

The PRIME code[47] solves the tearing mode equation[48] in a cylindrical geometry with circular concentric flux surfaces and conducting boundary. The code can calculate motion of flux surfaces and magnetic island width, given the magnitude of magnetic perturbations on the conducting boundary. 
The PEST code[49] is an ideal MHD stability code which has been in use at the Princeton Plasma Physics Laboratory as well as many research institutions around the world.

\subsection{Discharge Conditions}

Table 1 assembles in one place some plasma parameters of all discharges explicitly mentioned in this article. $B_{t}$ is the toroidal field at $R=R_{p}, \Delta_{s h}$ is the Shafranov shift of the magnetic axis, $q_{e d g e}$ is the magnetic twist parameter (safety factor) at the plasma edge, $\beta_{p}$ is the poloidal $\beta$, and $P_{b}$ is the injected NBI power. The tokamak produced discharges mainly at three different major radii, $R_{p} \approx 2.45 \mathrm{~m}, 2.52 \mathrm{~m}$, and $2.62 \mathrm{~m}$. Since the toroidal limiters limited the plasma on the inboard side at $R=1.65 \mathrm{~m}$, these three different major-radius discharges also represent three different minor-radius plasmas, $a_{p} \approx 0.80 \mathrm{~m}, 0.87 \mathrm{~m}$, and $0.97 \mathrm{~m}$. They will be referred to as small, medium, and large bore plasmas.

Table 1: Basic Discharge Parameters.

\begin{tabular}{|c|c|c|c|c|c|c|c|}
\hline $\begin{array}{c}\text { Shot \# } \\
\text { Units }\end{array}$ & $\begin{array}{c}B_{t} \\
\mathrm{~T}\end{array}$ & $\begin{array}{c}I_{p} \\
\mathrm{MA}\end{array}$ & $\begin{array}{c}R_{p} \\
\mathrm{~m}\end{array}$ & $\begin{array}{c}\Delta_{\text {sh }} \\
\mathrm{m}\end{array}$ & $q_{\text {edge }}$ & $\beta_{p}$ & $\begin{array}{c}P_{b} \\
\mathrm{MW}\end{array}$ \\
\hline$\# 65239$ & -4.49 & 1.39 & 2.62 & 0.22 & 7.35 & 0.80 & 17.9 \\
$\# 66869$ & -4.81 & 1.58 & 2.45 & 0.19 & 4.95 & 1.04 & 24.3 \\
$\# 73440$ & -4.68 & 1.78 & 2.52 & 0.18 & 4.92 & 0.88 & 22.8 \\
$\# 79983$ & -4.75 & 1.58 & 2.52 & 0.20 & 5.79 & 1.07 & 18.7 \\
$\# 81178$ & -4.50 & 1.78 & 2.62 & 0.16 & 5.67 & 0.56 & 23.0 \\
$\# 84522$ & -4.96 & 1.98 & 2.52 & 0.17 & 4.64 & 0.81 & 21.8 \\
$\# 84665$ & -4.57 & 0.99 & 2.62 & 0.16 & 10.2 & 0.58 & 4.5 \\
$\# 91560$ & -4.60 & 1.97 & 2.60 & 0.18 & 5.17 & 0.68 & 28.3 \\
$\# 91561$ & -4.60 & 1.97 & 2.60 & 0.18 & 5.17 & 0.68 & 25.9 \\
$\# 102287$ & -4.88 & 1.63 & 2.45 & 0.19 & 4.84 & 0.93 & 22.2 \\
\hline
\end{tabular}

\section{MAGNETIC PERTURBATIONS IN TFTR}

A main purpose of an analysis of magnetic perturbations is often to infer their spatial structure at an instant of time from their temporal behavior at discrete spatial locations. Experimental signals are usually too complex to carry out a meaningful examination directly in the time domain for this purpose, and require a Fourier analysis to isolate a specific frequency component. A natural tool for analysis is the discrete Fast Fourier Transform (FFT).

\subsection{Frequency Spectra}

Figure 4 shows a frequency spectrum (the modulus of an FFT as a function of frequency) of a signal from the Mirnov coil \#8(153/261) in a medium bore discharge \#73440. The sampling frequency of the signal was $500 \mathrm{kHz}$. The spectrum has two 
prominent peaks, one at $4.64 \mathrm{kHz}$ and another at $23.47 \mathrm{kHz}$, rising out of a 'noiselike background.' Another peak at twice the frequency of the lower frequency peak is also evident. These peaks are recognizable in all Mirnov signals for this discharge.

But magnetic perturbation spectra in TFTR are in general not similar around the poloidal circumference, even qualitatively speaking, as a spectrum for the Mirnov coil \#15(338/261) for the same discharge demonstrates in Fig. 5. The height of spectral peaks can vary by up to an order of magnitude-a main subject below. A noise-like background is stronger on the inboard side than on the outboard side by up to two orders of magnitude in many discharges.

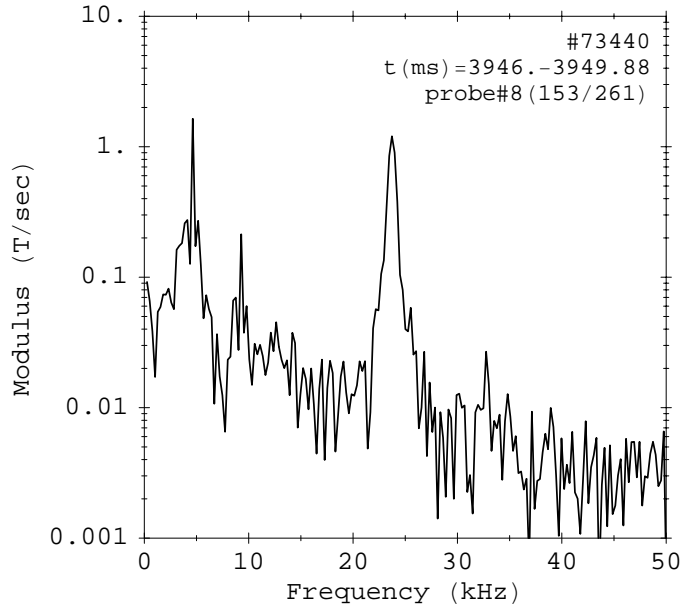

Fig. 4 Frequency spectrum of a Mirnov coil on the outboard side for shot \#73440. It has two prominent harmonic peaks. Compare with an inboard side signal in the next figure.

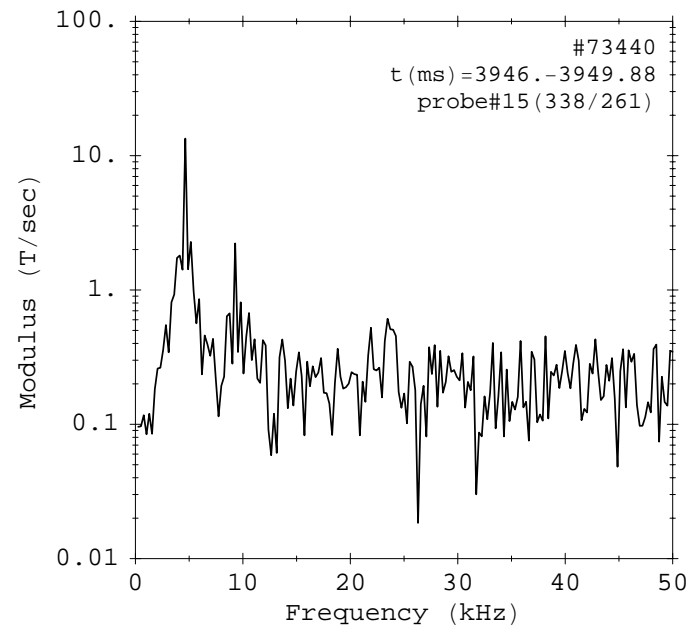

Fig. 5 Frequency spectrum of a Mirnov coil on the inboard side for shot \#73440. It has a single dominant peak. Compare with an outboard side signal in the previous figure.

\subsection{Gallery of Perturbation Patterns}

A simplistic view of an MHD mode in a tokamak is embodied in the formula: $\dot{B}_{i}(t)=A \cos \left(n \phi_{i}-m \theta_{i}-\omega t\right)$, where $\dot{B}_{i}(t)$ is the instantaneous signal intensity of an i-th Mirnov coil, 'A' is the signal amplitude, which is independent of the coil location, 'n' and 'm' are the toroidal and poloidal harmonic numbers, $\phi_{i}$ and $\theta_{i}$ are the toroidal and poloidal angles of the coil, and $\omega$ is the signal angular frequency.

We 'digitize' the signal given by the above formula, and take an FFT of the resultant time series. The modulus of the FFT is then a 'circle' when presented in a polar plot, and the argument is a 'straight line' when presented in a rectilinear plot as a function of $\theta_{i}$ for a given value of $\phi_{i}$. We will examine here the modulus and argument of an FFT of an experimental signal with this simplistic view in mind. Appendix A explains modifications to this simple picture encountered in a more realistic geometry. 


\subsubsection{Modulus Variation}

Figure 6 presents magnetic perturbations observed in six discharges. Each panel in the figure is in the form of a polar plot representing the poloidal variation of the modulus of an FFT in a discharge. A thick gray line is a six-degree polynomial curve fitted to the data points to guide the eye. The modulus is normalized to either maximum modulus found among all probes, or to a maximum value attained by the curve. The data points and the curve thus lie between zero (inner circle) and unity (outer circle).

Some perturbations have a modulus variation pattern that looks very different from a 'circle' expected in a simplistic picture of MHD modes. The difference motivated our present investigation as they may suggest the presence of a magnetic phenomenon other than MHD modes. We point out here some notable features of the patterns in the figure that appear in conflict with a simplistic view.

First, some patterns lack a symmetry with respect to the horizontal midplane (top right and bottom right). Moreover, some are strongly shifted toward the inboard side (top left and middle right), and some others are strongly shifted toward the outboard side (middle left and bottom left).

Second, the modulus varies poloidally by over an order of magnitude in all patterns. These variations can be like nodal points in a standing wave pattern (top right, middle left, and bottom right).

Third, some patterns have a group of modulus values that vary by up to a factor of two, though they are all at the same poloidal angle. Notice 'outliers' among NPA probes near the bottom of the curve in the lower left panel, which are spread over a moderately large range. Compare them with the same set of NPA points in the middle right panel, which are closely packed together. The data in both panels were from the same shot. They were taken at the same time during the discharge, but at different frequencies. We will distinguish the two sets of data here and in later figures by appending to the shot number a suffix, 'HF' (higher frequency), for the middle right panel and a suffix, 'LF' (lower frequency), for the bottom left panel. Notice also in the same pair of panels NPA probes near the inboard midplane. They are spread in different patterns between the two panels. A rotating MHD mode produces a modulus that is independent of the toroidal angle. The presence of the outliers is not consistent with the notion that an MHD mode alone produced the observed perturbations.

It is worthwhile to think about what kind of mechanism could possibly generate such outliers. The first important 'boundary condition' in this search for a candidate mechanism is that it generates outliers in one discharge but not in others, or, at one frequency but not at others even in the same discharge. A second condition is that the mechanism operates at the same frequency as the perturbations. It seems difficult to propose a plausible mechanism without bringing into the picture something that is external to the plasma-MHD-mode system and toroidally not 
axisymmetric.

Some 'moments' of a poloidal variation of the FFT modulus will be useful for a quantitative comparison. We define an 'in/out asymmetry' as,

$$
S_{i / o}=\frac{N_{\text {out }}}{N_{\text {in }}} \frac{\sum_{\text {in }} B_{i}}{\sum_{\text {out }} B_{i}}
$$

where $B_{i}$ is the FFT modulus of an i-th Mirnov coil signal, summation is over an arbitrarily chosen angular sector of $\pm 60 \mathrm{deg}$ about the inboard or outboard midplane, and $N_{\text {in }}$ and $N_{\text {out }}$ are the number of 'probe points' that fall within the respective sectors. (We will use many 'probe points' computed from a curve fitted to experimental data, rather than actual probe points, in order to obtain a better representation.) We will sometimes quote its reciprocal, an 'out/in asymmetry,' $S_{o / i}$, to avoid compressing a large variation between zero and unity. Analogous expressions define a yardstick for an 'up/down' or a 'down/up' asymmetry with angular sectors of the same width but now about a vertical direction. Numbers at the lower left corner of each modulus plot give the out/in and up/down asymmetry ratios.

\subsubsection{Argument Variation}

We now turn to the FFT argument in Fig. 7 for the same set of six discharges discussed in the previous figure. Each panel in the figure is a conventional rectilinear plot of the FFT argument. The abscissa is the poloidal angular location of the Mirnov coils, and the ordinate is the argument. The single filled square symbol is a duplicate entry of the probe\# 1 (shifted by $2 \pi$ along the abscissa and by $2 \mathrm{~m} \pi$ along the ordinate). Its inclusion emphasizes the periodic nature of the data set when fitting a curve.

The FFT argument of a signal from an NPA probe needs a correction to account for its toroidal displacement from that of PA probes. The correction to be subtracted from the argument is the product of an assumed toroidal harmonic number, 'n,' and the separation in toroidal angle, $\phi$, i.e., $n\left(\phi_{N P A}-\phi_{P A}\right)$. A 'coalescing' of PA and NPA probe arguments is in turn an indication that an assumed toroidal mode number was reasonable.

Experimental data appear to vary smoothly as a function of the probe angle in some patterns in the figure. A single curve is fitted to the data to guide the eye in these patterns. We used the Merezhkin angle given by Eq. A2. In other patterns data seem to have discontinuities, or 'jumps.' The data is divided into subsets in these discharges. Each subset is fitted with a third-degree polynomial individually. We point out some notable features of these argument plots. 

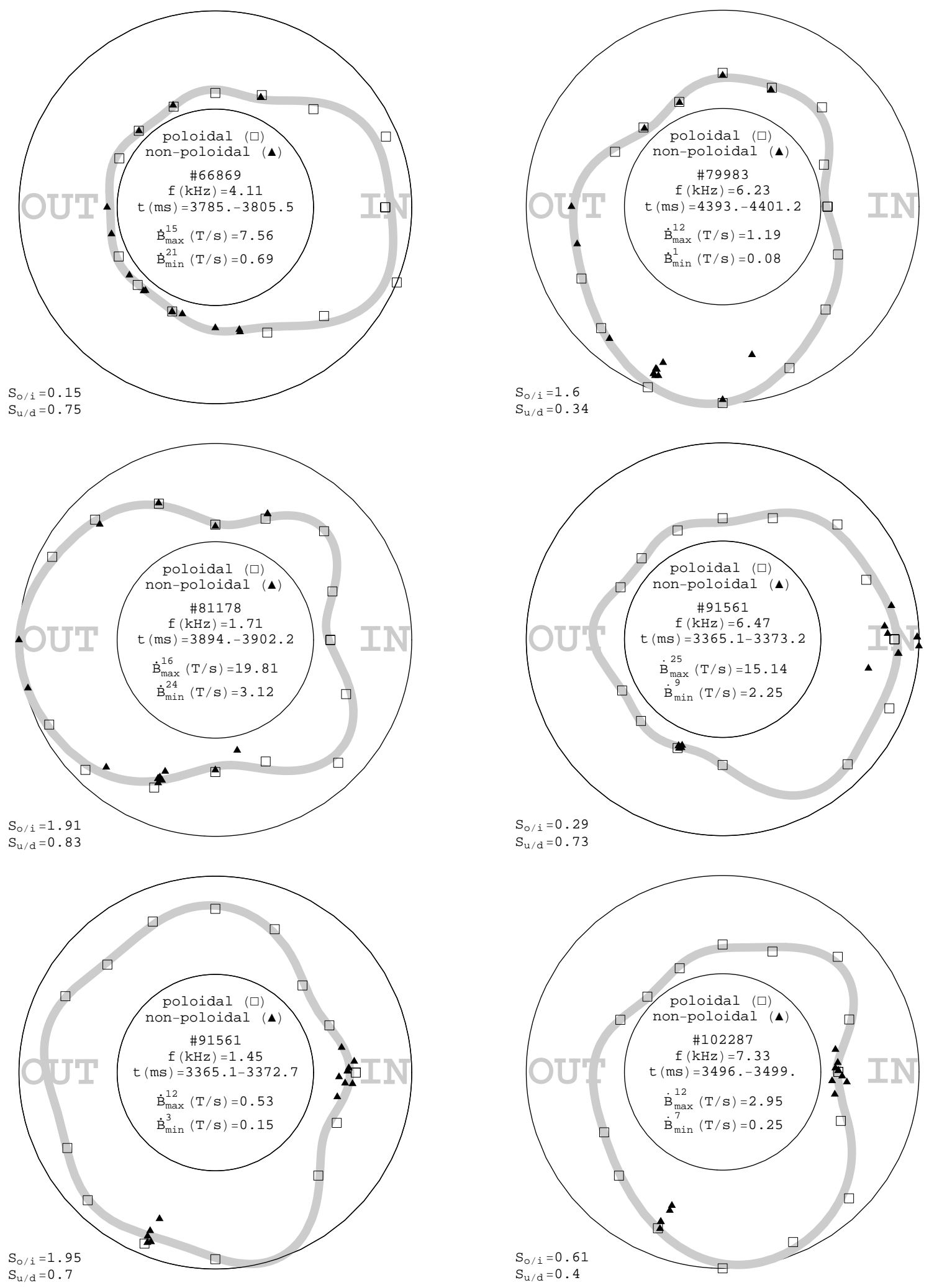

Fig. 6 Poloidal variation patterns of the FFT modulus for a set of six discharges. 

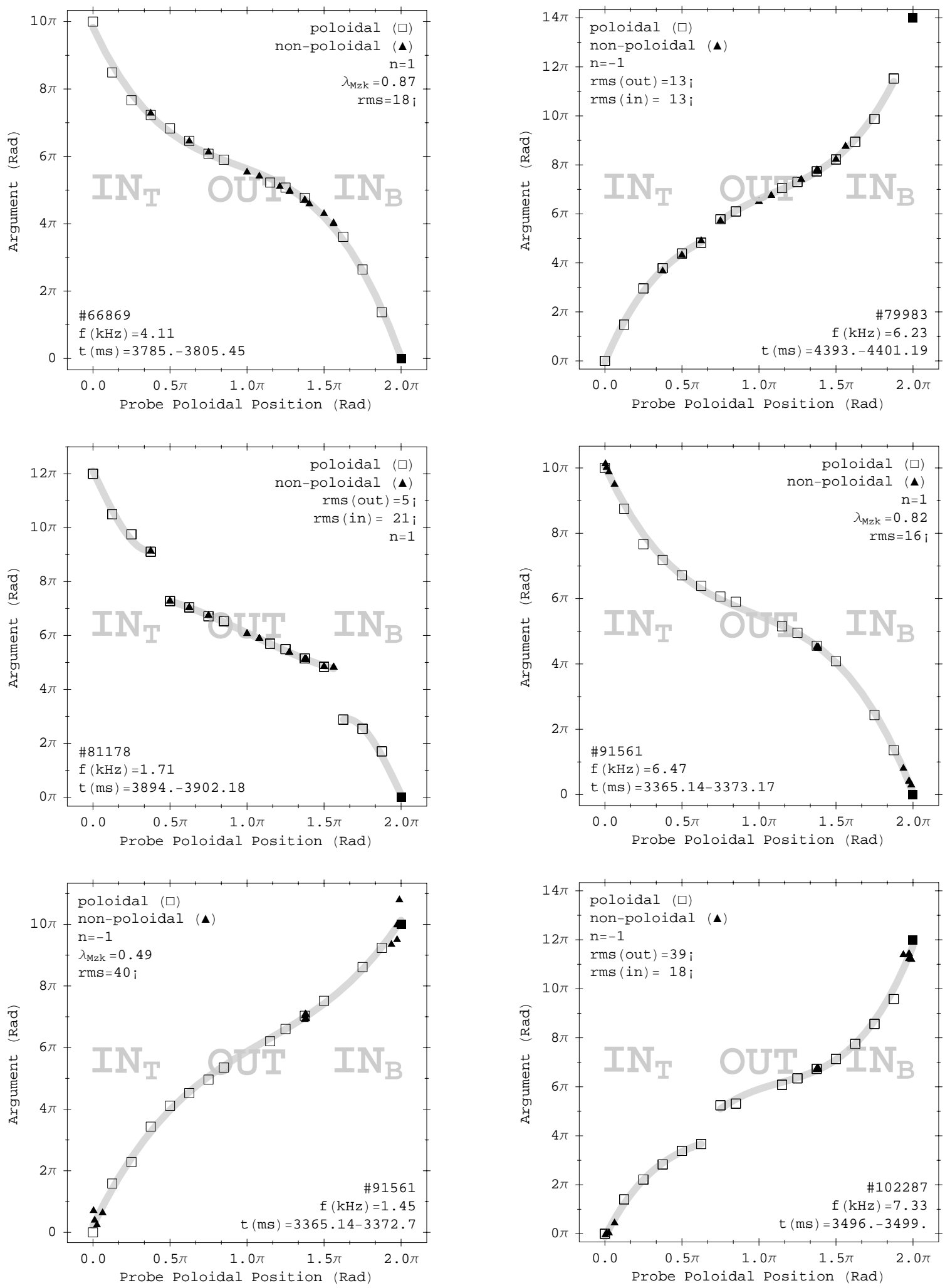

Fig. 7 Poloidal variation of the FFT argument for the same set of discharges shown in the previous figure. 
First, jumps exist, and occur at poloidal angles corresponding to node-like points in the modulus variation. The method of representing jumps has a consequence on determination of a total phase change, and hence the m-number, of perturbations. A set of rules is followed consistently in constructing an argument curve, albeit an arbitrary one. A jump is represented as an upward one in a curve trending generally upward, or as an downward one in a curve trending generally downward.

Second, the total variation in the argument, disregarding the sign, is (10-14) $\pi$, or $5-7$ when measured in units of $2 \pi$. The m-numbers, determined from the total phase variations, are thus close to $q_{\text {edge }}$ (see Table 1 ). But this assessment is uncertain for variations with jumps.

Third, PA and NPA probe points coalesce well with an $n=1$ assumption. A choice of any other n-number would cause NPA probe points to scatter widely. Thus, from a standpoint of the argument (phase), an $\mathrm{n}=1$ sinusoidal variation represents well the toroidal structure of observed perturbations in these shots as well as in other EOMP discharges studied in this article. Far fewer outliers are evident in the argument plots than in the modulus plots.

Fourth, the rotation direction of perturbation patterns in these discharges has little to do with the direction of torque imparted by NBI.

In this article's sign conventions an argument curve such as the one in the top right panel running from a lower left corner to an upper right corner represents a pattern with an $n=-1$ toroidal harmonic number. It rotates in a co-direction. An argument curve such as the one in the top left panel running from an upper left corner to a lower right corner represents $n=+1$. It rotates in a counter-direction.

All discharges in the figure had a NBI net torque in the co-direction, but at two distinct levels. Two discharges had a medium torque level (around +9 Newton$\mathrm{m})$. The perturbation pattern rotated in one direction in one of them, and in an opposite direction in the other. Four discharges had a low torque level (between +3 and +5 Newton-m). Rotation direction was also evenly split among them, two in one direction and the other two in an opposite direction. Two discharges not listed here, \#65239 and \#73440, had a high torque level (between +11 and +12 Newton$\mathrm{m})$ in the co-direction. The pattern in them also rotated in opposite directions.

The NBI net torque, total argument variation, and pattern rotation direction are listed in Table 2. Numbers for argument plots with jumps are enclosed in parentheses.

Table 2: Rotation Direction of Magnetic Perturbation Patterns.

\begin{tabular}{|r|c|c|c|c|c|c|}
\hline Shot \# & $\# 66869$ & $\# 79983$ & $\# 81178$ & $\# 91561 \mathrm{HF}$ & $\# 91561 \mathrm{LF}$ & $\# 102287$ \\
\hline torque $(\mathrm{N}-\mathrm{m})$ & +9 & +9 & +3 & +3 & +3 & +5 \\
total arg var & $-10 \pi$ & $(+14 \pi)$ & $(-12 \pi)$ & $-10 \pi$ & $+10 \pi$ & $(+12 \pi)$ \\
pattern rotation & $\mathrm{ctr}$ & $\mathrm{co}$ & $\mathrm{ctr}$ & $\mathrm{ctr}$ & $\mathrm{co}$ & $\mathrm{co}$ \\
\hline
\end{tabular}




\subsection{Closer Examinations}

The question is whether or not a set of magnetic perturbation patterns, consisting solely of MHD modes, can explain the observed modulus and argument variations. First, we will provide a quantifiable expectation from a realistic model for perturbations. Second, we will demonstrate that only a few MHD modes are actually available for re-constructing an observed pattern, and that they cannot reproduce on a quantifiable basis certain aspects of the pattern.

The first action relies on the VACUUM code. The code calculates a mathematically rigorous solution of the Maxwell's equations in a geometry sufficiently realistic to account for most important geometrical effects. The Appendix A describes this

process. The second comes from a direct inspection of potential sites for MHD modes and inherent properties of MHD mode eigenfunction.

TFTR has a simple geometry: a plasma of a circular cross section and a vacuum vessel also of a circular cross section. It therefore has less complicated plasma physics and boundary conditions than shaped plasma tokamaks. Its internal structures, e.g., limiters, are also relatively transparent to magnetic perturbations. This gives us a measure of confidence in interpreting differences between observations and expectations on magnetic perturbation patterns. It would be a far more arduous task to make a similar study in a shaped plasma tokamak.

\subsection{Constituent Harmonics}

\subsubsection{Data Analysis Approach}

Given an arbitrary set of poloidal harmonics at our disposal, we can reconstruct any poloidal variation through superposition. However, poloidal harmonics of an MHD mode must (1) reflect symmetry property of an unstable system from which they arise; MHD modes of an up/down symmetric system are expected to possess an up/down symmetry, (2) overlay each other with specific relative spatial phases, and (3) originate on relevant singular surfaces; they have definite major and minor radii in a given q-profile.

These constraints will help to limit the number of constituent harmonics. For example, a hypothesis that $\mathrm{n}=1$ perturbations came only from MHD modes will limit potential sites for their origin to integer q-value singular surfaces. We will examine these sites, and eliminate any of them that fail to show evidence of actual existence of perturbations. The temperature fluctuation at a site sets maximum amplitude of that constituent.

A poloidal harmonic becomes an actual constituent when direct evidence is found, or a potential constituent when its site is inaccessible for inspection, for example, a site beyond a GPC viewing domain. The elimination process may leave only a single constituent harmonic, or a few at most. 
Perturbation amplitude originating at these constituent harmonic sites, both actual and potential, will be transformed to a pattern on a Mirnov circle using VACUUM code calculations. The Appendix A summarizes calculation result. A minimal set of constituent harmonics will be able to produce a pattern with only a limited range of characteristics. We will examine the range to see whether a set of constituent harmonics could qualify as the source of an observed perturbation pattern.

\subsubsection{Interpretations of Individual Discharges}

We will examine the six discharges in Figs. 6 and 7 in light of the VACUUM code calculations. The top half of Table 3 summarizes experimentally observed characteristics of perturbations in these discharges.

The n- and m-numbers are from the argument plots. An m-number from an argument curve with jumps is enclosed in parentheses. The Merezhkin coefficient obtained in two different ways is listed: $\lambda_{M z k}^{f i t}$ is from fitting to argument data, and $\lambda_{M z k}^{c a l}$ is calculated using Eq. A3. A discharge with an argument plot with jumps has only a calculated value. The calculations used the inverse aspect ratio of the last integer singular surface in the plasma.

The down/up and out/in asymmetry ratios are from the modulus plots. Another row lists an inferred displacement in $\mathrm{cm}$, if the down/up asymmetry were to be interpreted as caused by a vertical displacement of the plasma. The conversion comes from Fig. 43, Appendix A.

A common trait in many discharges of our present interest is the absence of internal fluctuations in plasma interior regions at the same frequency, and with the same helicity, as external magnetic perturbations: either no fluctuations were detected, or they were detected but with a helicity incompatible with external perturbations.

The first row in the bottom half of Table 3 with a heading, 'interior pert,' contains information obtained from an analysis in later sections. It indicates the presence of electron temperature fluctuations in plasma interior regions. A 'no' answer means the absence of fluctuations at the same frequency as external magnetic perturbations. A 'yes' answer, however, means the presence of fluctuations at the same frequency, but irrespective of their helicity compatibility. We chose this criterion, because jumps in an argument plot sometimes make the m-number of external magnetic perturbations uncertain. A 'yes' answer means the existence of one or more actual constituent harmonics. But on the basis of m-numbers cited in the same table, all 'yes' cases had a helicity (surmised from a q-value at the location of fluctuations) incompatible with external perturbations.

The second row in the bottom half of the table lists potential constituent harmonics. These are also integer q-value singular surfaces in edge regions, but outside of the GPC viewing domain. 
Our goal in this section is to motivate a more critical look at low frequency magnetic perturbations in TFTR, and suggest the possible need for reaching outside the traditional realm of MHD theory to explain them. It will thus suffice to show that some features of these patterns cannot be produced by a set of actual and potential harmonics, if they were to have originated from MHD modes with their attendant constraints.

Table 3: Features of Perturbations

\begin{tabular}{|r|c|c|c|c|c|c|}
\hline Shot \# & $\# 66869$ & $\# 79983$ & $\# 81178$ & $\# 91561 \mathrm{HF}$ & $\# 91561 \mathrm{LF}$ & $\# 102287$ \\
\hline plasma bore & small & medium & large & semi-large & semi-large & small \\
n from arg fit & +1 & -1 & +1 & +1 & -1 & -1 \\
m from arg fit & +5 & $(-7)$ & $(+6)$ & +5 & -5 & $(-6)$ \\
$\lambda_{M z k}^{\text {fit }}$ & 0.87 & N/A & N/A & 0.82 & 0.49 & N/A \\
$\lambda_{M z k}^{\text {cal }}$ & 0.85 & 0.94 & 0.75 & 0.84 & 0.84 & 0.80 \\
down/up & 1.3 & 2.9 & 1.4 & 1.4 & 1.4 & 2.5 \\
out/in & -1.5 & -10 & -2 & -2 & -2 & -9.4 \\
inferred disp (cm) & 0.15 & 1.6 & 2.0 & 0.29 & 2.0 & 0.63 \\
\hline interior pert. & no & no & yes & no & yes & yes \\
potential m's & 3,4 & 6 & 4,5 & 5 & 5 & 4 \\
\hline
\end{tabular}

Up/Down Asymmetry - An observation common to all patterns is the presence of an up/down asymmetry of a varying magnitude, although all discharges were centered on the midplane within a few $\mathrm{mm}$. From an up/down asymmetry viewpoint the perturbations observed in all of these discharges were inconsistent to a varying degree with a notion that they originated only from an MHD mode.

This holds, unless the plasma itself was up/down asymmetric with respect to the device midplane. We used guidance from VACUUM code calculations to assess the likelihood of the existence of such a position asymmetry. Shots \#79983 and \#102287 are particularly striking examples of the up/down asymmetry. But the plasma would have to be below the midplane by around $10 \mathrm{~cm}$ to explain its magnitude. Carbon light emission in shot \#79983 from a pair of points symmetrically located about the midplane on the bumper limiters indicated that this discharge was well balanced vertically. From this independent confirmation in addition to a magnetics diagnostic, it is unthinkable that the plasma was unintentionally off the midplane by such a distance.

The up/down asymmetry can take also another form. The existence of two nodelike points in the modulus variation in shot \#79983 suggests that it represented a superposition of a pair of poloidal harmonics with a harmonic number difference of two. (Two-harmonic superposition is discussed in the Appendix A.)

But these harmonics cannot both be MHD modes. First, only a single potential constituent harmonic existed (see 'potential m's' row in Table 3). At least one, or possibly both, of the pair must be something other than an MHD mode. Second, the nodal points, one near the inboard midplane and another around $135 \mathrm{deg}$, were 
not symmetrically disposed about the midplane, and could not have been produced by superposition of two MHD modes, each with a symmetric pattern. Again, at least one, or possibly both, of the pair must be something other than an MHD mode.

The modulus variation in shot \#102287 had similar features as shot \#79983 just discussed. The present shot had an actual constituent harmonic in interior regions and a potential constituent harmonic in edge regions. But the nodal points, one near the inboard midplane and another around $135 \mathrm{deg}$, were again not symmetrically disposed about the midplane, and could not have been produced by superposition of two MHD modes, each with a symmetric pattern. Again, at least one of the constituents must be something other than an MHD mode.

The qualitative nature of the contradiction in the up/down asymmetry offers a strong reason for concluding that a magnetic phenomenon other than MHD modes was present in these discharges.

In/Out Asymmetry - An MHD mode must originate from a singular surface relevant to its helicity. The specific geometry of that surface places restrictions on the kind of patterns that an MHD mode can produce on a Mirnov circle. By applying the VACUUM code result from the Appendix A to potential constituent harmonics listed in the Table 3, we can judge in some cases whether or not these harmonics were indeed responsible for generating the observed pattern.

The perturbation pattern in shot \#79983 had an observed out/in asymmetry of 1.6. But the discharge had a single potential constituent harmonic, $\mathrm{m} / \mathrm{n}=6 / 1$. It is expected to have an out/in asymmetry ratio of 0.1 according to the VACUUM code analysis (Appendix A, Fig. 41 - point 'S' of the medium bore curve). It cannot match the observation on a quantitative basis.

The observed $\mathrm{m} / \mathrm{n}=5 / 1$ pattern in shot $\# 91561 \mathrm{LF}$ had an out/in asymmetry ratio of 2.0. No single harmonic in any size plasma can produce such a value. Generating such a pattern with superposition of two harmonics would require the presence of an $\mathrm{m}=4$ or 6 harmonic. An out/in asymmetry ratio generated by the two-harmonic effect would still be at most about 1.3, and still falls well short of the observed value (Appendix A, Fig. 45). Besides, the observed interior perturbations were mostly confined within the $\mathrm{q}=1$ surface, and the actual constituent harmonic thus had $\mathrm{m}=1$. It could not have been a needed partner in the two-harmonic superposition.

The observed $\mathrm{m} / \mathrm{n}=5 / 1$ pattern in shot \#66869 had an in/out asymmetry ratio of 6.7 (reciprocal of the value, 0.15 , cited in the Table 3 ). It was much smaller than a value, 15.2, expected for an MHD mode of this helicity lying in the edge region of a small bore plasma (Appendix A, Fig. 41).

Two potential constituent harmonics, $\mathrm{m}=3$ and 4 , are not likely to explain this discrepancy. First, their helicity is not compatible with the observations. (The determination of the m-number was probably quite reliable, because of a good 
quality of the argument fit and the absence of jumps.) Second, the observed pattern shows little indication that it is a superposition of two harmonics with a unity m-

number difference. Superposition of such harmonics with comparable amplitudes would produce a deep node-like structure at the inboard midplane (Appendix A, Fig. 45), which is not evident in the observed pattern.

Merezhkin Effect - All discharges except \#91561LF had a large value of the Merezhkin coefficient. It is expected to produce a prominent indentation, or a 'dimple,' at the inboard midplane (Appendix A, Fig. 49). Shots \#79983, \#81178, and \#102287 had a feature that could be interpreted as a dimple. But neither \#66869 nor \#91561HF shows much indication of its presence.

Complex Situation - The discharge \#81178 had internal temperature fluctuations at the same frequency as external magnetic perturbations. Its modulus and argument variations are representative of discharges with well developed islands in the vicinity of integer singular surfaces (often at $q=2$, but here at both $q=2$ and 3 ) detected by internal diagnostics. The discharge was included here for that reason, but represents a more complex situation for analysis. It has two potential constituent harmonics, $\mathrm{m}=4$ and 5 .

Its argument variation suggests that external magnetic perturbations had an $\mathrm{m} / \mathrm{n}=+6 /+1$ pattern when interpreted in a standard way on the basis of a total variation. But jumps made the interpretation uncertain. The slope of a center segment of the argument curve (outboard side) is in fact compatible with an $\mathrm{m} / \mathrm{n}=+2 /+1$ variation. But edge segments (inboard side) have a much steeper slope (larger m-value). Jumps occur at juxtapositions of the two disparate slopes.

Effort is often futile for reproducing an observed pattern in this and other similar discharges by varying the relative amplitudes of harmonics. Note that relative phases are not a free parameter, if these harmonics are to be regarded as MHD modes. Superposition of patterns, in a manner consistent with the hypothesis of MHD modes, reproduces gross features, but not many important details such as locations of nodes and relative amplitudes of lobe-like structures. With an increasing number of constituent harmonics, such effort can be neither exhaustive nor conclusive. But an impression from such an effort is usually that at least one of the patterns must be able to produce an amplitude variation different over parts of poloidal circumference, usually a variation with a larger amplitude on the outboard side.

\subsection{Section Summary}

We have exploited TFTR's simple geometry and the VACUUM code's ability for a realistic representation of the device in interpreting differences between observations and expectations on a magnetic perturbation pattern on a Mirnov circle. Such interpretations help to determine whether or not a set of MHD modes alone could have produced that pattern. 
This section contributes the following major conclusions with respect to a class of magnetic perturbations in TFTR that can be characterized by a toroidal harmonic number of $n=1$ and a poloidal harmonic number comparable to an edge q-value of the discharge.

1. A magnetic phenomenon originating in edge regions exists having a poloidal amplitude variation that is more general than is expected for an MHD mode. We will call the phenomenon by a short-hand name, 'Edge Originated Magnetic Perturbations (EOMP's).'

2. EOMP's manifest their presence in an observed perturbation pattern:

(a) In a qualitative, and hence most unambiguous, manner as an up/down asymmetry; it is evident in averaged modulus values as well as disposition of node-like points when they are present.

(b) In a more quantitative manner, as an in/out asymmetry unachievable by a combination of MHD modes that are actually observed internally, and undetected but potentially present.

(c) Presumably also in a symmetric manner.

(d) As 'outliers' (data points that do not fit in the poloidal variation trend) in the modulus and argument plots.

3. EOMP's produce:

(a) Down/up asymmetry ratio up to 3.0 in a vertically centered discharge.

(b) Out/in asymmetry ratio up to 2.0 for a large bore plasma, which in its absence is expected to have an in/out asymmetry ratio up to 3.1 .

\section{THREE CLASSES OF PERTURBATIONS}

This section has a broader aim than the last one. We will attempt to break down diverse magnetic perturbation patterns observed in TFTR into a few basic building blocks. The first task is to identify basic classes of perturbations. A second task is to find each class's defining characteristics.

A class of magnetic perturbations called EOMP's has already emerged in the preceding section. Two more classes, Kink-Like Modes (KLM's) and Tearing Modes (TM's), will appear in this section. The KLM has two sub-classes. Our primary focus is still EOMP's. We want to show how they fit in with other classes of perturbations.

We will seldom find an individual class in its entirely pure form. But we can characterize it sufficiently in a 'single class event,' where a single class is dominant, though not necessarily alone. We will select such discharges to aid our characterization effort. 
We will examine three aspects of internal temperature fluctuations, beginning from 'raw' data and moving on to more highly processed information. We will also examine two aspects of external magnetic perturbations. These examinations will help to distinguish among the four classes and sub-classes.

But cross-referencing information among a $5 \times 4$ matrix of combinations requires some effort. The following table of figure numbers is for facilitating the ease of comparison. The first row lists the classes. The second row gives the sub-classes and shot numbers of discharges used as the representative of each class. The headings in the first column are key words from discussion that will follow. The entry in parentheses under 'Mod/Arg Poloidal Var' and EOMP refers to the same source as the one under 'KLM with EOMP.'

Table 4: Figure Cross References among Classes

\begin{tabular}{|r|c|c|c|c|}
\hline Class & TM & \multicolumn{2}{|c|}{ KLM } & EOMP \\
\hline Shot & $\# 84665$ & $\begin{array}{c}\text { with EOMP } \\
\# 91561 \mathrm{LF}\end{array}$ & $\begin{array}{c}\text { with TM } \\
\# 66869 \mathrm{HF}\end{array}$ & $\# 91560$ \\
\hline$T_{e}$ Profile & 8 & 9 & 10 & 11 \\
Phase Reversal & 12 & 13 & 14 & 15 \\
Buried Activity & 16 & 17 & 18 & 19 \\
Mod/Arg Poloidal Var & 20,21 & 6,7 & 22,23 & $(6,7)$ \\
Mod Contour & N/A & 26 & 24 & 25 \\
\hline
\end{tabular}

\subsection{Oscillating Temperature Profiles}

We examine major-radial profiles of the electron temperature at two closely spaced times to discern differences among the classes. The profile times are apart by one half of a cycle of oscillations at the frequency of the most prominent spectral peak in the electron temperature fluctuations. When an electron temperature spectrum lacks a prominent peak, we use a magnetic perturbation spectrum.

Electron temperature data are from GPC-1. The sampling frequency was $500 \mathrm{kHz}$ except where noted. Mapping from frequency to major radius uses total magnetic field computed by the TRANSP code, and introduces a small correction by requiring that electron temperature be equal on a flux surface (usually a $q=1$ surface) in an unperturbed or a time-averaged profile. Figure numbers for the four cases are under the heading, ' $T$ e Profile,' in Table 4.

\subsubsection{TM's}

Figure 8 presents electron temperature profiles for a TM event (\#84665). The temperature oscillated at $7.38 \mathrm{kHz}$. The phase of oscillations showed a 'reversal' (explained in the next sub-section) between signals from adjacent channels (see Fig. 12). Such a phase reversal indicates the presence of a flat spot in a temperature 
profile produced by the magnetic island of a tearing mode. The two profiles in the figure are at the times of arrival in front of the instrument of the ' $\mathrm{x}$ '-point and ' $\mathrm{o}$ 'point of an island, respectively. The 'o'-point profile has a flat spot at the location of each phase reversal. It is shown in a manner compatible with a measurement made at discrete radial points. An island appeared at the same instance of time on either side of the magnetic axis at $278.9 \mathrm{~cm}$, indicating that the fluctuations had an even m-number structure. A q-profile computed by the TRANSP code located a $\mathrm{q}=2$ surface at $245.4 \mathrm{~cm}$ and $310.0 \mathrm{~cm}$, just outside (larger minor radius side) of these islands. Magnetic perturbations at the same frequency had an $(\mathrm{m} / \mathrm{n})=(-2 /-1)$ structure (see Fig. 21). These were therefore likely to be $(\mathrm{m} / \mathrm{n})=(-2 /-1)$ islands.

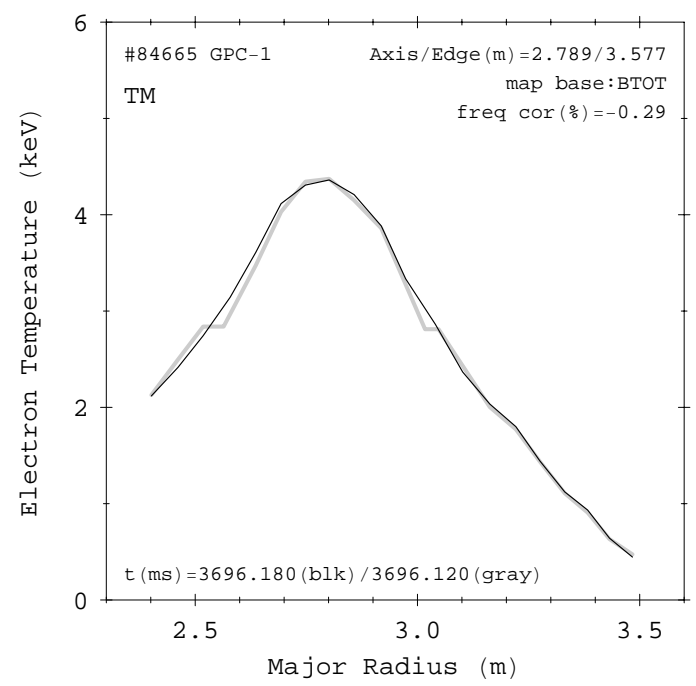

Fig. 8 Electron temperature profiles at two closely spaced times in shot \#84665 - a TM event. It had $(\mathrm{m} / \mathrm{n})=(-2 /-1)$ tearing mode islands. The black and gray profiles are for the times of arrival in front of the GPC-1 instrument of an ' $\mathrm{x}$ '-point and an 'o'-point of an island, respectively.

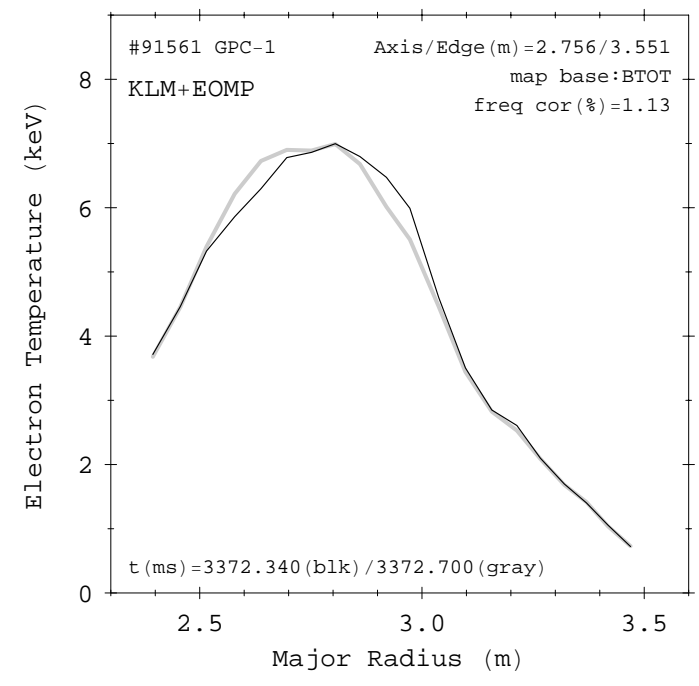

Fig. 9 Electron temperature profiles at two closely spaced times in shot \#91561LF - a KLM event with an EOMP companion. It had an odd-m KLM (probably $(\mathrm{m} / \mathrm{n})=(-1 /-1))$ and an EOMP. The black profile is most 'outward tilting' and the gray one the most 'inward tilting' within a cycle of oscillations.

The inboard (smaller major radius side) island occupied a radial interval, 251.8$256.4 \mathrm{~cm}$, with a width of $4.6 \mathrm{~cm}$ and at a temperature of $2839 \mathrm{eV}$. The outboard island was between $301.7-304.8 \mathrm{~cm}$, with a width of $3.1 \mathrm{~cm}$ and at a temperature of $2812 \mathrm{eV}$. An average of the inboard and outboard island widths was $3.9 \mathrm{~cm}$. These are islands of a modest size. Well-developed islands in TFTR are typically twice as large in terms of the width, but they almost always appear with strong perturbations of the other classes. This discharge was selected, because it is a single class event, and more suitable for characterization of the class. The q-profile predicted a $q=2$ singular surface that was farther out in minor radius than the observed island locations by several centimeters. The discrepancy may be traceable to difficulties in accurately calculating flat central regions of a q-profile. A slight difference in temperature between the inboard and outboard islands may be a result of fluctuations at other frequencies. The maximum value of measured magnetic perturbations among all probes was $0.29 \mathrm{~T} / \mathrm{sec}$ at $7.45 \mathrm{kHz}$ (perturbation field of 
$64 \mathrm{mG}$ )(see Fig. 20).

The PRIME code computed the island width for the TM event in shot \#84665. We will describe procedures for calculations in a later section. The code used a maximum measured magnetic perturbation strength as a boundary condition, and obtained a value of $2.8 \mathrm{~cm}$. It is $28 \%$ smaller than the observed average width of $3.9 \mathrm{~cm}$. Recall that the TRANSP calculated q-profile located $\mathrm{q}=2$ surfaces further out in minor radius than the observed islands. The discrepancy in the island size may in part be a result of a difference in $q^{\prime}$ at the computed singular surface locations and the actual island locations. We consider that the agreement is reasonable, and that in this single class event for a TM the observed internal fluctuations and observed external perturbations are consistent with each other in magnitude.

\subsubsection{KLM's}

A KLM is a phenomenon that exhibits internal fluctuations of clearly recognizable characteristics. But external magnetic perturbations observed at the time of the appearance of a KLM are similar to those of either an EOMP or a TM. A KLM appears to be always accompanied by a companion.

Two profiles in Fig. 9 show a KLM event (\#91561LF) with an EOMP companion. The electron temperature oscillated at $1.58 \mathrm{kHz}$. The fluctuations were at their extreme phases when the profiles were taken: the black profile at the most 'outboard tilting' position and the gray at the most 'inboard tilting' position within a cycle of oscillations. The phase of oscillations showed no reversal (see Fig. 13). The lack of a phase reversal is consistent with fluctuations without flux surface tearing, such as an ideal kink mode. During each cycle of oscillations a central part of the profile moved back and forth between the extreme positions. The motion, which is a defining characteristic of KLM's, is revealed clearly in this figure. It represented most likely toroidal rotation of a helical kink-like distortion assumed by a central part of the plasma.

A q-profile calculated by the TRANSP code placed the magnetic axis at $275.6 \mathrm{~cm}$, and the $\mathrm{q}=1$ surface at $243.7 \mathrm{~cm}$ and $305.5 \mathrm{~cm}$. Most of the motion thus occurred within the $\mathrm{q}=1$ surface. Oscillations persisted beyond the surface. But their amplitudes waned rapidly toward the plasma edge. We will show this more clearly in a later section (see Fig. 17).

A kink mode at the $\mathrm{q}=1$ surface should have an $(\mathrm{m} / \mathrm{n})$ ratio of unity. For fluctuations with $n=-1$ it should be an $(m / n)=(-1 /-1)$ kink mode. The largest signal among all Mirnov probes was $0.53 \mathrm{~T} / \mathrm{sec}$ at $1.45 \mathrm{kHz}(0.58 \mathrm{G})$, and their spatial structure was $(\mathrm{m} / \mathrm{n})=(-5 /-1)$ (see bottom left panel of Fig. 7). A slight discrepancy $(130 \mathrm{~Hz})$ in the peak frequency between temperature and magnetic measurements is within the FFT frequency resolution. But the spatial structures appear to be in a serious conflict with each other. We will address this helicity incompatibility a 
few paragraphs down, but describe another case of KLM first.

A KLM event (\#66869HF) with TM magnetic perturbations is shown in Fig. 10. The temperature oscillated at $16.9 \mathrm{kHz}$. The two profiles again represent two extreme positions of a fluctuating structure. The temperature fluctuations exhibited a phase reversal (see Fig. 14) near the $\mathrm{q}=3 / 2$ surface predicted in TRANSP calculations. (But for simplicity data points in the figure are this time connected by straight line segments to form profiles, and no flat spots are indicated.) The external magnetic perturbations had a spatial structure of $(\mathrm{m} / \mathrm{n})=(-3 /-2)$ (see Fig. 23). The maximum strength of perturbations measured among all probes was $4.8 \mathrm{~T} / \mathrm{sec}$ at $16.9 \mathrm{kHz}(0.45 \mathrm{G}$ ) (see Fig. 22). A tearing mode of the same helicity therefore probably caused the phase reversal. In a combination of a KLM and a TM the two members must have the same n-number in order for them to maintain a constant relative phase to each other over many cycles of oscillations. Furthermore, the location of the KLM at a $q=1$ surface dictates that its $m$ - and n-numbers are the same. The KLM in the present event thus had an $(\mathrm{m} / \mathrm{n})=(-2 /-2)$ structure.

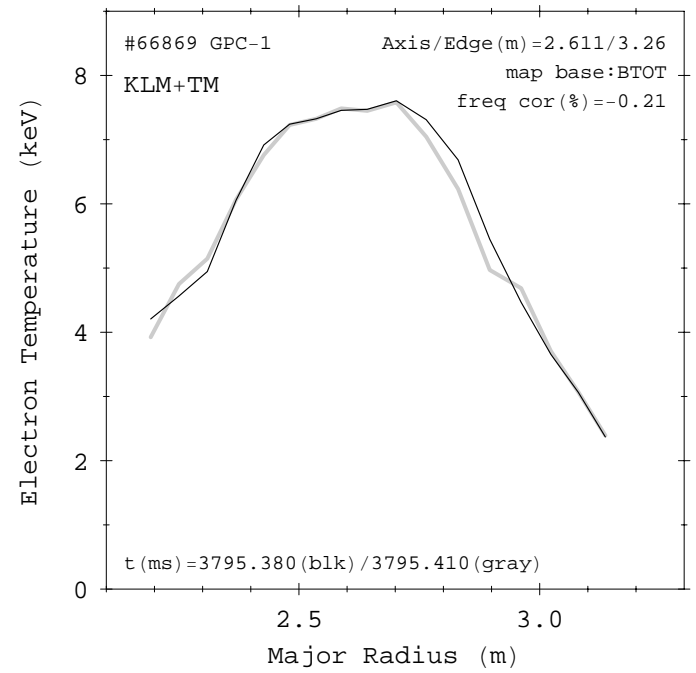

Fig. 10 Electron temperature profiles at two closely spaced times in shot \#66869HF - a KLM event with a TM companion. It exhibited both KLM features with an even m-number (probably $\mathrm{m}=2$ ) and TM features with an odd $\mathrm{m}$ number (probably $\mathrm{m}=3$ ) in the plasma interior: the black and gray profiles are for the times of arrival in front of the GPC-1 instrument of an ' $\mathrm{x}$ '-point and an 'o'-point of an island, respectively.

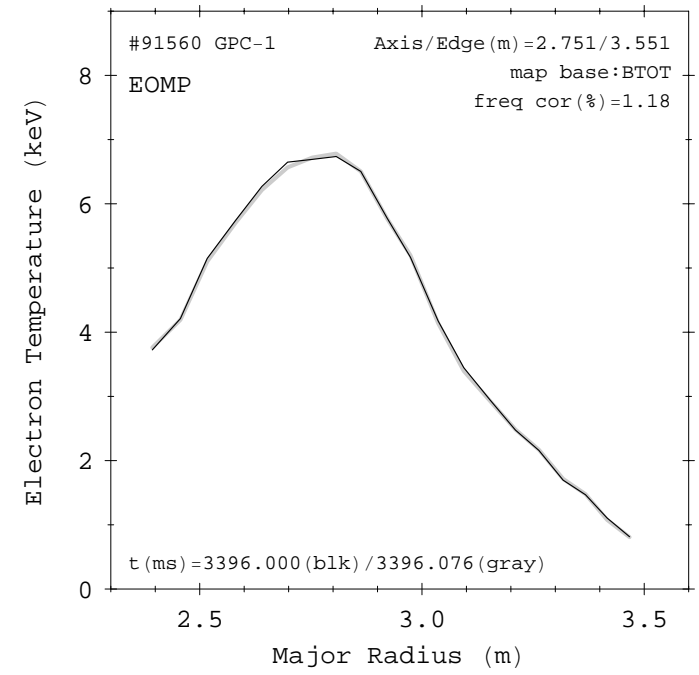

Fig. 11 Electron temperature profiles at two closely spaced times in shot \#91560 - an EOMP event. It had no detectable organized perturbations in the plasma interior: the profile times are apart by one half period of external magnetic perturbations $(6.59 \mathrm{kHz})$ observed at the same time.

We return to the helicity incompatibility question, and offer two possible explanations. The first one proposes that a KLM appears always accompanied by an EOMP, a TM, or both, and that it possesses relatively small external magnetic perturbations of its own. It is the companion's magnetic perturbations that dominate the KLM's, and become detected externally. This model is consistent with the two KLM events just discussed above. 
An alternative explanation is to invoke theoretical expectations for an external kink mode. It is a global mode. An eigenfunction corresponding to an eigenvalue, say, $\mathrm{n}=1$, has perturbations that vary globally over a poloidal cross section. We may choose to expand its poloidal variation into a Fourier series. Then it has a set of harmonics, $1 / 1,2 / 1,3 / 1$, etc., up to an m-value somewhat larger than $q_{\text {edge. }}$. One might say that the origin of the instability is in the plasma core region, but 'a set of different m-number modes are coupled to the plasma edge.' This explanation says that observed external magnetic perturbations came from a large m-number member of the set. A KLM is solely responsible for them, and has no need to invoke the presence of a companion.

This ideal mode model runs into a few problems. It attributes the observed magnetic perturbations solely to an MHD mode. They can thus have only patterns that are allowed for MHD modes. A discharge (\#102287) described earlier in Figs. 6 and 7 had also a KLM. Its modulus variation had a strongly up/down asymmetric pattern, which is not expected for MHD modes. PEST code calculations also showed that the first KLM discharge (\#91561LF) discussed was stable against $\mathrm{n}=1$ kinkmodes without conducting walls. For these reasons we favor the 'KLM with a companion' model over the 'coupled m-number ideal mode' model.

A KLM event exhibits external magnetic perturbations. It also has ideal-modelike internal fluctuations. We note that these are also necessary ingredients for an RWM. They are parts of the same physical phenomenon in the case of an RWM. But they come from separate sources in the case of a KLM, and do not necessarily obey the same causality relationship.

Relative Phase - We examine how two modes, a KLM and a TM, orient themselves and fit in with each other spatially when they appear in the same discharge.

Returning to the discharge, \#66869HF, just discussed in Fig. 10, the 'parity' of the m-number is opposite between the two members: the TM has an odd m-number, $(\mathrm{m} / \mathrm{n})=(-3 /-2)$, and the KLM has an even m-number, $(\mathrm{m} / \mathrm{n})=(-2 /-2)$. Fluctuations from the two members thus reinforce each other on one side of the magnetic axis and annihilate on the other. The difference produces a characteristic fluctuation structure evident in the figure. On the outboard side the two profiles run together, generating a 'parallel lines' feature between a flat-top edge at $R_{m a j} \sim 272 \mathrm{~cm}$ and an island at $R_{m a j} \sim 295 \mathrm{~cm}$. But on the inboard side the profiles cross each other at $R_{m a j} \sim 235 \mathrm{~cm}$, resulting in a 'crossed lines' feature.

This characteristic structure has been seen in the same orientation in many discharges. For example, shot \#73440(4000) had an $(\mathrm{m} / \mathrm{n})=(-4 /-3)$ TM and a ($3 /-3)$ KLM, i.e., the m-number parity opposite from the case discussed above - the number in parentheses following a shot number is the time of interest in millisecond. But the discharge had a 'parallel-line' feature also on the outboard side.

These observations indicate that two fluctuation structures of different m-numbers, one a KLM mode and the other a TM, fit to each other in a specific relative spatial orientation. 


\subsubsection{EOMP'S}

Temperature profiles of an EOMP event (\#91560) are shown in Fig. 11. Their time points were apart by one half cycle of observed magnetic perturbations. The profiles are nearly identical to each other, indicating the absence of detectable organized fluctuations in the interior regions at the frequency of observed magnetic perturbations. They must then have come from sources in the edge regions. We will examine exhaustively in the next section the issue of the absence of detectable organized fluctuations in an EOMP.

External magnetic perturbations had a structure quite similar to that of \#91561 shown in Fig. 6, middle right panel. But we have selected here \#91560 instead, because it was an EOMP event - without perturbations of other classes at any frequencies over the period of interest. (The shot \#91561 had a KLM and an EOMP at two different frequencies.)

The maximum amplitude of perturbations measured among all probes was $12.4 \mathrm{~T} / \mathrm{sec}$ at $6.59 \mathrm{kHz}(3.0 \mathrm{G})$. This was by far the strongest external magnetic perturbation among the three classes discussed here. Yet, the interior regions were devoid of detectable organized temperature fluctuations. In contrast, the TM (\#84665 with $64 \mathrm{mG}$ ) and the KLM with a TM companion (\#66869HF with $0.45 \mathrm{G}$ ) discussed above had magnetic perturbations smaller by a factor of about fifty and seven, respectively, than the EOMP here, but showed clearly recognizable internal fluctuations.

\subsection{Phase Reversal}

We will now look at only a fluctuating part of the electron temperature. Phase reversal and spatial localization of the fluctuating part will provide distinguishing characterization for the three classes.

Subtracting from each GPC temperature signal its time-averaged value over a short time window will leave the fluctuating part of the temperature. Figures 1215 show it for individual channels of GPC-1 for the examples of the three classes introduced in the previous subsection. These figures are in a 'multigraph' format. Traces are displaced along the ordinate by an arbitrary amount to avoid overlapping, but are ordered by their major radii, the smallest at the bottom and the largest at the top of the figure. Each trace has its channel number indicated beside it along the right-hand edge of the figure. The amplitude of each trace is 'auto-scaled' to fill the allotted space, and a number in parentheses following the channel number is the maximum peak-to-peak value attained by the trace over the time window. Arrows along the left-hand edge of the figure show 'locations,' with respect to the traces, of the magnetic axis ('m.a.') and a few relevant singular surfaces. Two vertical lines, labeled ' $a$ ' and ' $b$,' indicate the time points for the temperature profiles in the corresponding figures shown earlier. Figure numbers for the four cases are under the heading, 'Phase Reversal,' in Table 4. 


\subsubsection{TM's}

The TM event (\#84665) is in Fig. 12. A coherent oscillation was most evident in channel 17 on the inboard (smaller major radius) side and channel 10 on the outboard side. On the inboard side the phase of fluctuations was clearly opposite between channels 17 and 18. On the outboard side a corresponding 'phase reversal' occurred between channels 8 and 9 , but was less evident on account of the relative smallness of fluctuations as well as a greater noise-like component. Such a phase reversal can occur when a 'flat spot' produced by a magnetic island exists between adjacent channels: temperature rises (relative to a time averaged value) on the outer (larger minor radius) side, while it falls on the inner side, of the island, as its ' $\mathrm{O}$ '-point arrives in front of the measuring instrument; temperature variations in an opposite direction occur upon arrival of an ' $x$ '-point. In other words, an 'o'-point arrives wherever adjacent traces tend to 'merge' on the inboard side and 'diverge' on the outboard side; an ' $x$ '-point arrives when the traces behave in an opposite direction. Gray ellipses over the traces 17 and 18 and between traces 8 and 9 indicate one of such 'o'-point arrivals. The organized activity was spatially limited to the island neighborhood on the inner side of a $q=2$ surface. The phase reversal is the most distinctive feature of a TM. The absence of an evident reversal does not preclude islands. But such islands are by definition 'undetectably small.' We will explain this phrase more quantitatively in the next section.

\subsubsection{KLM's}

An event involving a KLM (\#91561LF) demonstrates its internal characteristics best with an EOMP as a companion, because the latter adds no complications to event's internal structure. This is evident in Fig. 13. An organized activity was largest on the inner (smaller minor radius) side of the $\mathrm{q}=1$ surface, but extended beyond it at much reduced amplitudes (note amplitude scales). The phase of fluctuations was the same for all traces on either side of the magnetic axis. It did not exhibit any reversals other than an expected one across the axis. Localization of fluctuations within a $\mathrm{q}=1$ surface and the absence of a phase reversal are principal defining features of a KLM.

Fluctuations shown in this figure also exhibit 'bursting' and 'whistling' characteristics: the amplitude builds up and then falls over several cycles of oscillations while the frequency changes. The behavior is reminiscent of the 'fishbone' instability. But neither 'bursting,' nor 'whistling,' is a distinguishing feature of a KLM. We see in other discharges bursting fluctuations evolving into continuous oscillations without losing the features of a KLM.

The KLM event with a TM companion (\#66869HF) discussed earlier is in Fig. 14. The three outboard-most traces are missing here because they are either dead or contaminated by non-thermal emission for this discharge. A slower sampling frequency $(100 \mathrm{kHz})$ and a much shorter time window $(0.5 \mathrm{~ms})$ both contributed to 
the difference in appearance of these traces.

A KLM event with a TM companion is expected to be essentially a sum of a KLM event with an EOMP companion and a TM event, because an EOMP adds little to an internal fluctuation structure. But the plot in Fig. 14 does not quite look like a sum of plots in Figs. 12 and 13. A primary reason for the betrayed expectation is simply that, unlike the plots in those figures, here the KLM has an even m-number, and the TM has an odd m-number. Also, fewer traces inside the $\mathrm{q}=1$ surface show oscillations here in comparison with the previous KLM example with an EOMP companion. But this is a simple reflection of a nearly flat central temperature profile in this discharge where flux surface displacement produces little temperature variations (compare profiles in Figs. 9 and 10).

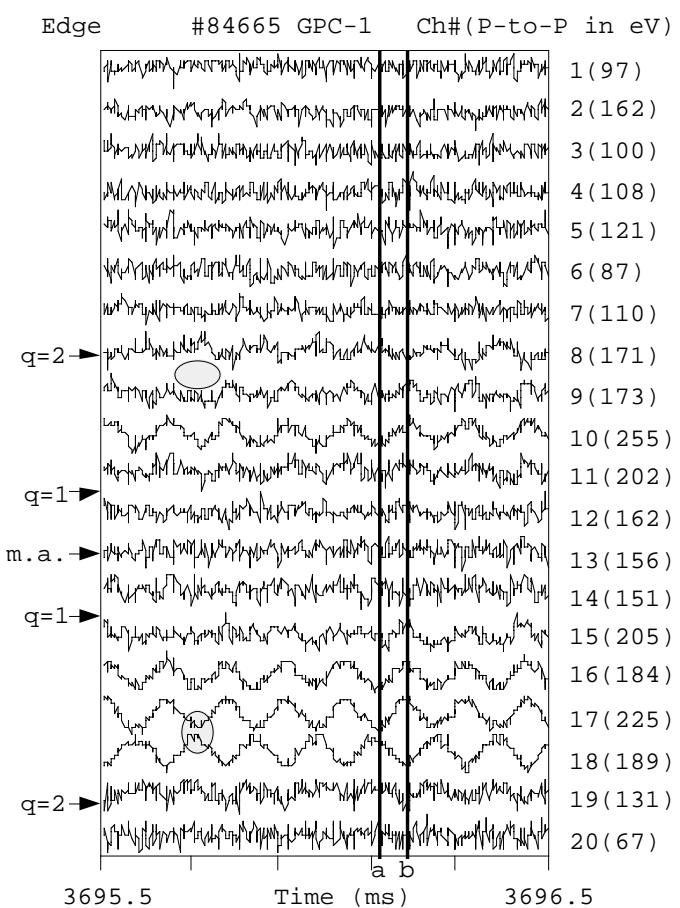

Fig. 12 Fluctuating part of the electron temperature in shot \#84665 - a TM event. Traces are displaced to avoid overlapping. The channel number is on the right-hand side of each trace followed by its amplitude in parentheses.

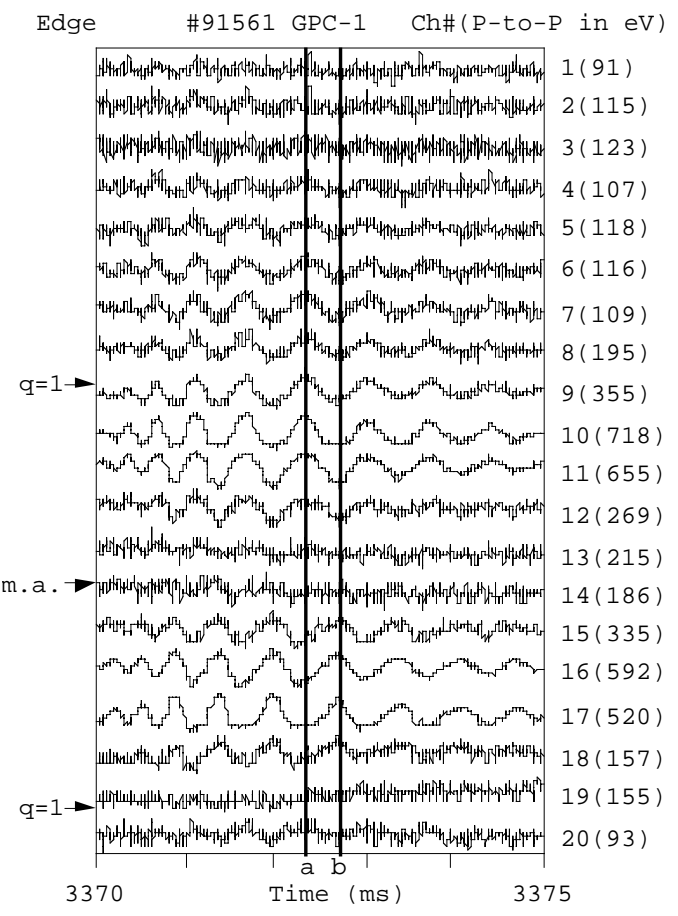

Fig. 13 Fluctuating part of the electron temperature in shot \#91561LF - a KLM event with an EOMP companion. For explanation of the figure structure, see the previous figure.

Temperature changes with an 'even-m symmetry' are evident near the $q=1$ surface. The temperature rises or falls together, for example, at the traces \#10 and \#16. On the outboard side fluctuations undergo a phase reversal between the traces \#7 and \#8 across the outboard $(\mathrm{m} / \mathrm{n})=(-3 /-2)$ island. On the inboard side the fluctuation structure is more complex. Temperature variations in opposite polarities between the KLM and the TM produces a 'node' as well as a phase reversal near the trace \#17. The fluctuations then undergo another phase reversal between the traces \#19 and \#20 across the inboard $(\mathrm{m} / \mathrm{n})=(-3 /-2)$ island. 


\subsubsection{EOMP'S}

The EOMP event (\#91560) distinguishes itself in not having any detectable organized activity in the interior regions. This is evident in Fig. 15. Finer scale fluctuations were ubiquitous on all traces here (as well as in the other events). They did not exhibit an obvious organized activity, at least to the 'naked eye.' But could it somehow be buried in the noise-like fluctuations? The next subsection will address this subject.

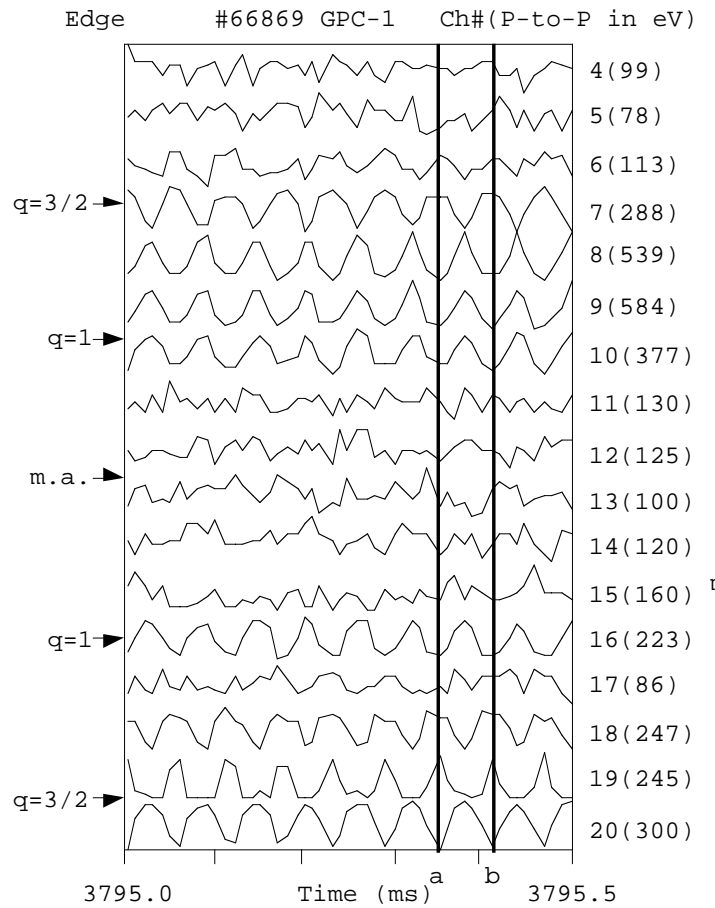

Fig. 14 Fluctuating part of electron temperature in shot \#66869HF - a KLM event with a TM companion. For explanation of the figure structure, see three figures earlier.

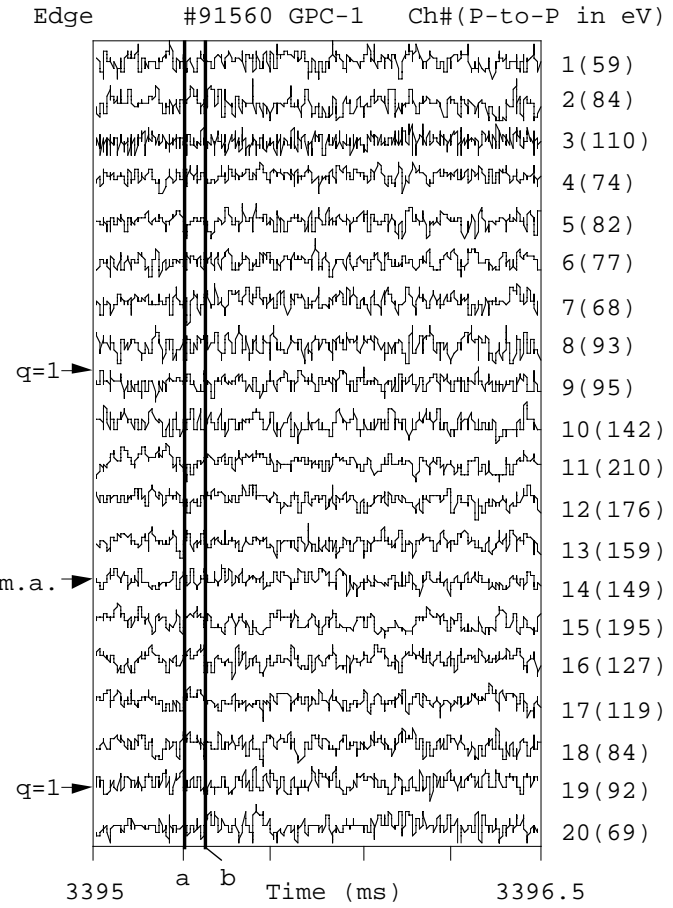

Fig. 15 Fluctuating part of electron temperature in shot \#91560 - an EOMP event. For explanation of the figure structure, see four figures earlier.

\subsection{Buried Organized Activity}

A Fourier analysis should be able to discover organized activity masked by noise. We mean activity that has some coherence in frequency and a spatial scale length not too large in comparison with the plasma minor radius. Displaying temperature fluctuations as a contour plot in a space-frequency domain will be useful in finding such activity.

We first explain how we construct such a plot. An FFT of GPC temperature data at each major-radius is taken over a sub-interval of a fast sampling window. The fluctuating component at each frequency and radius is normalized by the time averaged temperature at that radius to yield a set of fractional temperature fluctu- 
ation data. A contour plot is constructed from it over a GPC viewing domain and a frequency range of interest.

We would expect an organized activity to emerge as a set of nested closed contours. Contours at a level meandering over a wide range of frequency suggests noise-like activity at that level. Contours covering a wide range in space represents a global 'rigid body-like' change. By this rather vague criterion the noise level appears to be somewhere below $0.2 \%$. Contours at this level will be shown in a gray line in a plot. We will delve into the notion of noise more quantitatively in a later section (Sec. 5.2.4).

The outboard limit of a GPC viewing domain usually does not reach the plasma boundary, either because a particular instrument setting used does not allow for full coverage, or because contamination from non-thermal emission degrades signals from channels in the plasma edge regions. Typically, the last $10 \%$ of plasma minor radius is not covered. But this varies from one discharge to another. Edges of contour lines mark the limit of the viewing domain in a contour plot. A gray horizontal line is sometimes drawn there also. Arrow markers along the left-hand edge of a plot will indicate locations of the magnetic axis ('m.a.'), singular surfaces, and plasma boundary ('p.b.'). Figure numbers for the four cases are under the heading, 'Buried activity,' in Table 4.

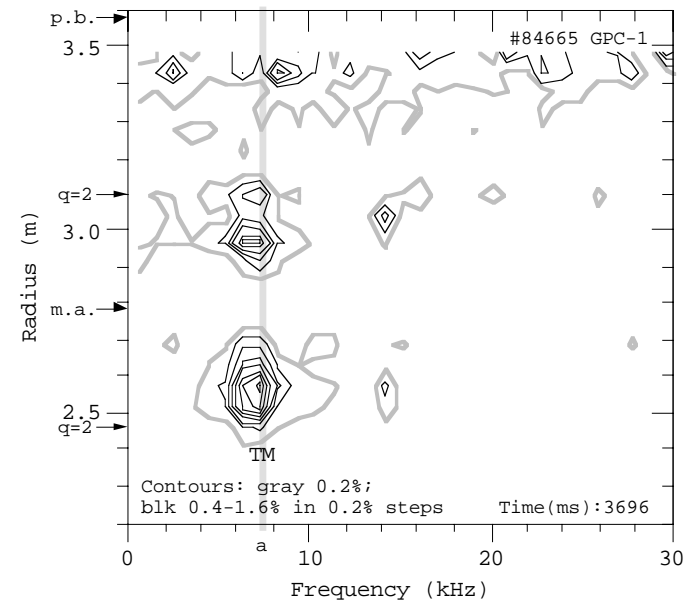

Fig. 16 Contours of equal FFT modulus values of fractional $T_{e}$ perturbations in shot \#84665 a TM event.

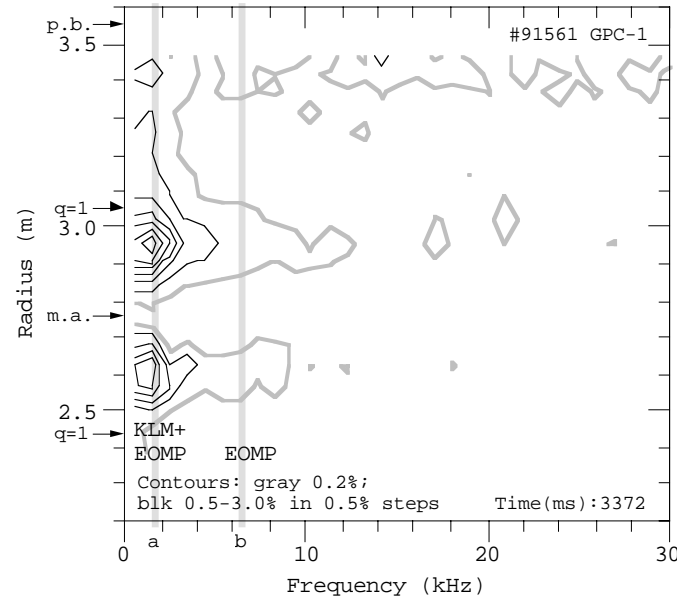

Fig. 17 Contours of equal FFT modulus values of fractional $T_{e}$ perturbations in shot \#91561LF - a KLM event with an EOMP companion.

\subsubsection{TM's}

How will the magnetic islands of the TM event discussed earlier appear in such a contour plot? For shot \#84665 in Fig. 16 they are two 'blobs' of nested closed contours in a range, $6-8 \mathrm{kHz}$, and radial locations approximately on the inner (smaller minor radius) side of the $\mathrm{q}=2$ markers. Contours in black begin at $0.4 \%$, and progress at $0.2 \%$ intervals. The tallest peak at $1.6 \%$ is in the inboard side 'blob.' 
A gray vertical line, 'a,' indicates the frequency of the most prominent spectral peak at $7.38 \mathrm{kHz}$ of magnetic perturbations. The viewing domain extends up to $348.4 \mathrm{~cm}$ while the plasma boundary is at $357.7 \mathrm{~cm}$, covering $90 \%$ of the $96.4 \mathrm{~cm}$ minor radius.

Groups of nested contours, each indicating possible organized activity, are evident at a number of frequencies along the outboard boundary of the viewing domain. We will call these groups 'clumps,' and discuss them later in an EOMP section.

\subsubsection{KLM's}

The KLM event (\#91561LF) with an EOMP companion appears in Fig. 17. The viewing domain and plasma edges are at $347.1 \mathrm{~cm}$ and $355.1 \mathrm{~cm}$, respectively. The domain covers $92 \%$ of the $95.1 \mathrm{~cm}$ minor radius. Gray vertical lines, 'a' and 'b,' are at $1.59 \mathrm{kHz}$ and $6.47 \mathrm{kHz}$, which are frequencies of secondary and primary spectral peaks of magnetic perturbations, respectively. Two 'blobs' occur along the gray line, 'a,' and represent the KLM with an EOMP companion. The black contours begin at $0.5 \%$ and progress in $0.5 \%$ steps. The tallest peak at $3.0 \%$ is in the outboard blob. Note the absence of any detectable organized activity along the gray line, 'b,' i.e., at the frequency of the primary magnetic spectral peak. This is the frequency of a 'stand-alone' EOMP. The relationship between the EOMP and the KLM with an EOMP companion in this discharge will become clearer later (see Fig. 26).

Figure 18 shows the other KLM event (\#66869) with a TM companion. With some missing channels the viewing domain extends up to $313.6 \mathrm{~cm}$ while the plasma boundary is at $326 \mathrm{~cm}$, covering only $84 \%$ of the $80 \mathrm{~cm}$ minor radius. A gray vertical line, 'a,' is at $16.89 \mathrm{kHz}$, which is the frequency of a primary spectral peak of magnetic perturbations. Two 'blobs' occur along the gray line, and represent the combination of a KLM and a TM. The black contours begin at $0.5 \%$ and progress in $0.5 \%$ steps. The tallest peak at $3.0 \%$ is in the outboard blob. The small viewing domain is probably a main reason why no clumps are visible. Notice a gray vertical line, 'b,' at $4.11 \mathrm{kHz}$, which is the frequency of a secondary spectral peak of magnetic perturbations. This is the frequency of a 'stand-alone' EOMP. The relationship between the EOMP and the KLM with a TM companion in this discharge will become clearer later (see Fig. 24).

\subsubsection{EOMP's}

Figure 19 demonstrates that the EOMP event (\#91560) looks strikingly different (data also shown in Fig. 15). The viewing domain covers $91 \%$ of the $95.1 \mathrm{~cm}$ minor radius. The domain and plasma edges are at $346.6 \mathrm{~cm} 355.1 \mathrm{~cm}$, respectively. The gray line, 'a,' at $6.60 \mathrm{kHz}$ is the frequency of a dominant spectral peak of magnetic perturbations. Black contours start at a lower $0.3 \%$ value, and progress in smaller

$0.1 \%$ steps. Except perhaps for a hint of a feeble KLM, no organized activity was 
evident in the interior regions of the plasma. Clumps were still evident at a number of frequencies, but amplitudes of their visible parts were no greater here than in the other events. A peak fluctuation value anywhere within the viewing domain was only $0.6 \%$, and attained in a clump at the primary spectral peak frequency.

The question of whether or not this particular clump had anything to do with the EOMP under discussion can be dealt with directly by examining its temporal coherence. A contour plot (not shown) similarly constructed from an edge GPC-1 channel in the frequency-time domain (rather than the frequency-radius domain) demonstrates that the particular clump was a short-lived phenomena (several millisecond), and therefore had little to do with the EOMP, which persisted over most of the NBI heated period (see Fig. 25).

Clumps are the only detectable organized temperature fluctuations in an EOMP event. Contours in many of them are open at the boundary, suggesting that their main bodies lie further outboard. The visible part of any of these clumps has a small amplitude. But is it possibly a 'tip of an iceberg' that lies beyond the viewing domain boundary? What is the largest tearing mode island that can hide in this narrow strip of the last $10 \mathrm{~cm}$ of the plasma? The next section (Sec. 5) will address these questions.

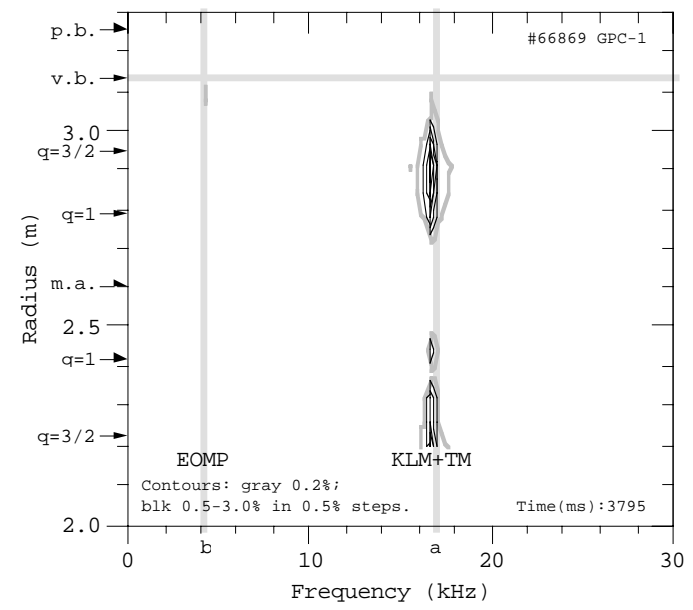

Fig. 18 Contours of equal FFT modulus values of fractional $T_{e}$ perturbations in shot \#66869HF - a KLM event with a TM companion.

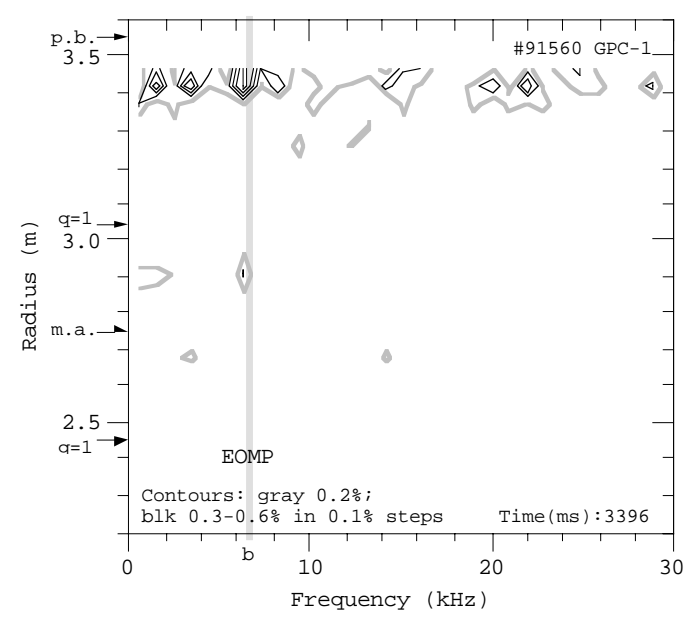

Fig. 19 Contours of equal FFT modulus values of fractional $T_{e}$ perturbations in shot \#91560 an EOMP event.

\subsection{External Appearance}

We now return to matters of external magnetic perturbations. We have so far looked at only the EOMP's presented earlier in Figs. 6 and 7. We examine here whether the external magnetic perturbations of the other classes also look so different from a simplistic view as EOMP's. Figure numbers for the four cases are under the heading, 'Mod/Arg Poloidal Var,' in Table 4. 


\subsubsection{TM's}

We might expect that a TM event has the best chance to come close to simple expectations from an MHD mode, because it is after all the only class among the three that has an established theoretical basis. Modulus and argument variations are in Figs. 20 and 21 for the TM event (\#84665). The maximum modulus occurred at the probe \#11(248/261), and was $0.29 \mathrm{~T} / \mathrm{sec}$ at $7.45 \mathrm{kHz}(64 \mathrm{mG})$.

The result is fairly close to simple expectations in many respects. The modulus variation is substantially 'circular.' An argument analysis indicates an $\mathrm{n}=-1$ toroidal structure and a total variation of $-2 \pi \mathrm{m}$ with $\mathrm{m}=-2$. This is consistent with an earlier observation of islands at the $\mathrm{q}=2$ surface. The magnetic perturbations rotated in the same sense as the net torque exerted by NBI, which was +7.1 newton-m in a co-direction.

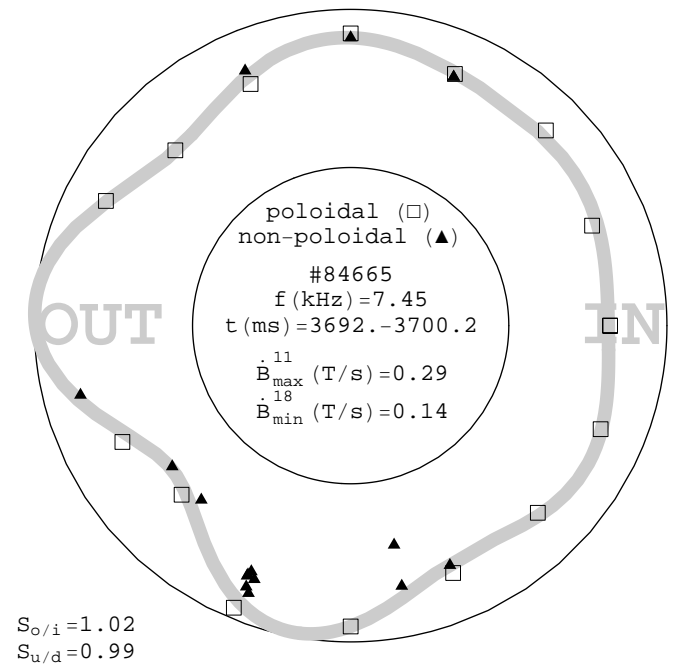

Fig. 20 Modulus of the FFT of magnetic perturbations for shot \#84665 - a TM event.

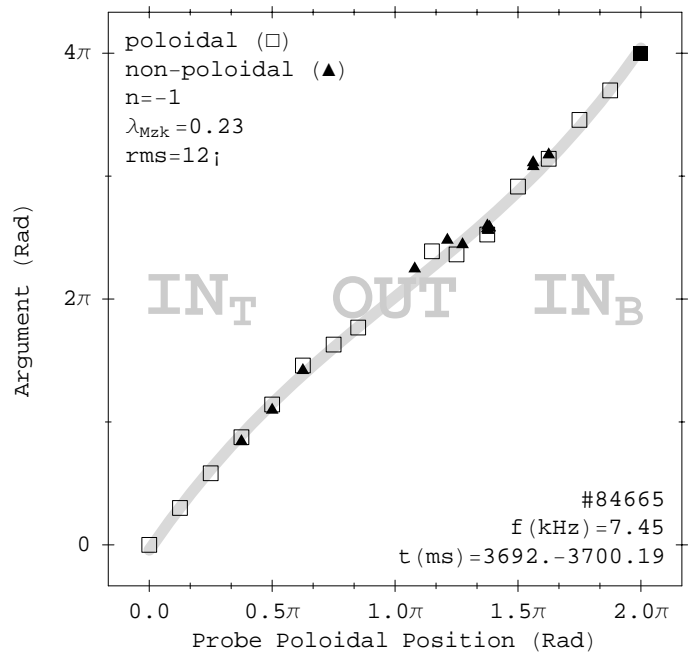

Fig. 21 Argument of the FFT of magnetic perturbations for shot \#84665 - a TM event.

Some anomaly is also evident, however. Outliers exist in both modulus and argument plots. The modulus variation also has a pentagonal deformation with pronounced 'dents' on its outboard faces. A moderate EOMP present at the same frequency might have produced them.

The measured in/out asymmetry ratio was 0.98. According to the Appendix A, Fig. 40, a $\mathrm{q}=2$ singular surface in a large bore plasma would have an in/out asymmetry ratio of 0.94 . In making this comparison we ignored the fact that the calculated result was for a different large bore plasma. But it is probably not too bad a comparison, because the $\mathrm{q}=2$ singular surface was centered at similar radii $-277.7 \mathrm{~cm}$ for the present discharge (\#84665) and $275.0 \mathrm{~cm}$ for the calculations (\#81178). The minor radius was significantly different, $34.2 \mathrm{~cm}$ for the present discharge and $57.8 \mathrm{~cm}$ for the calculations. But recall that the aspect ratio of the source circle was much less important in determining the out/in asymmetry (Appendix A). Note also that the measured up/down asymmetry ratio was 0.99 , quite close to an expected 
value of unity.

\subsubsection{KLM's}

Modulus and argument variations are in Fig. 22 and 23 for the KLM event (\#66869HF) with a TM companion. The maximum modulus occurred at the probe \#14(315/261), and was $4.8 \mathrm{~T} / \mathrm{sec}$ at $16.87 \mathrm{kHz}(0.45 \mathrm{G})$.

This event nearly reproduces simple expectations for MHD modes. The modulus variation is almost 'circular,' and has no significant outliers. An argument analysis produces a good fit with an RMS error of only $10 \mathrm{deg}$. The analysis indicates an $\mathrm{n}=-2$ toroidal structure and a total argument variation of $-2 \pi \mathrm{m}$ with $\mathrm{m}=-3$. This is consistent with an earlier observation of islands at a $q=3 / 2$ surface (see Figs. 14 and 18). The magnetic perturbations rotated in the same sense as the net torque exerted by NBI, which was +8.3 newton- $\mathrm{m}$ in a co-direction at the time of the event.

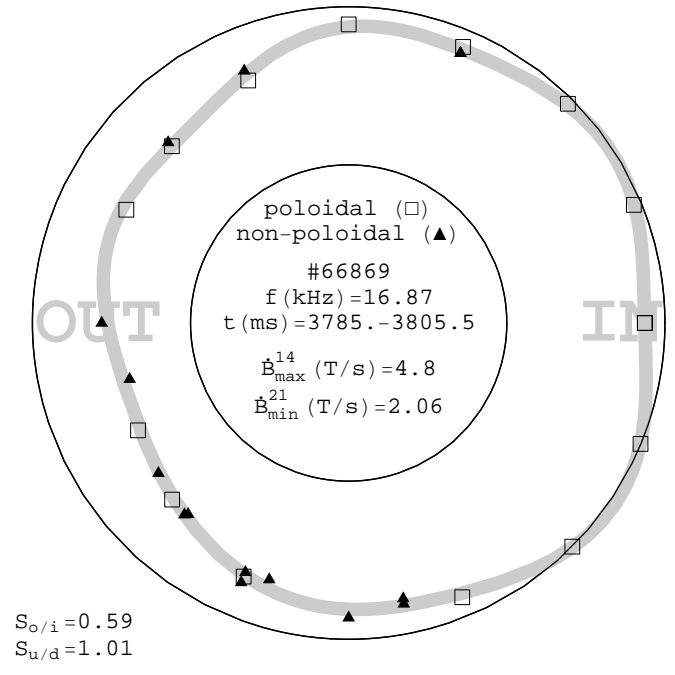

Fig. 22 Modulus of the FFT of magnetic perturbations for shot \#66869HF - a KLM event with a TM companion.

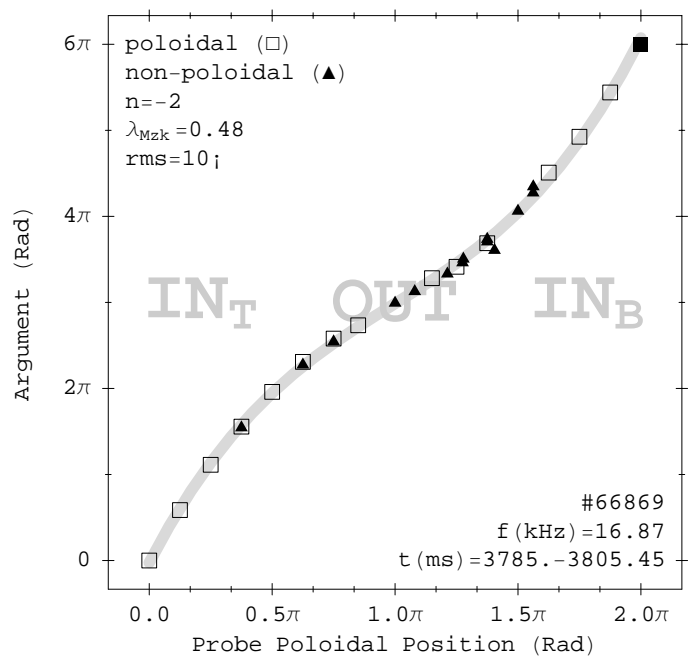

Fig. 23 Argument of the FFT of magnetic perturbations for shot \#66869HF - a KLM event with a TM companion.

The observed in/out asymmetry ratio was 1.69. Rather than relying on Fig. 40, we must calculate the in/out asymmetry anew for this discharge as it has an $n=2$ toroidal harmonic. The calculated value is 2.22 . The observed in/out asymmetry is significantly smaller $(24 \%)$ than the expectation. We also see that the observed poloidal pattern is significantly 'taller' than the calculated one. We do not understand the origin of the discrepancy at this point.

The modulus and argument variations already appeared in Figs. 6 and 7 (\#91561LF) for the KLM event with an EOMP companion that has been under discussion in Figs. 10, 14, and 18 in this section. A maximum modulus occurred at the probe \#12(270/261), and was $0.53 \mathrm{~T} / \mathrm{sec}$ at $1.45 \mathrm{kHz}$ (0.58 G). Sec. 3.4 already discussed these variations. 


\subsubsection{EOMP'S}

The modulus and argument variations of the EOMP event (\#91560) will not be shown here, because they are similar to those of the EOMP event (\#91561HF) already discussed in Figs. 6 and 7 (middle right panel in either figure). The maximum modulus in the present shot occurred at the probe \#25(358/135), and was $12.41 \mathrm{~T} / \mathrm{sec}$ at $6.59 \mathrm{kHz}(3.0 \mathrm{G})$.

\subsection{Spectral Time Evolution}

We have focused so far nearly exclusively on an instantaneous view of these events. A contour plot in a frequency-time domain is a good means to show their time evolution. It shows how these events relate to each other when they occur in the same discharge at the same time but at different frequencies. Figure numbers for the three cases are under the heading, 'Mod Contour,' in Table 4 (plot for the TM case - \#84665 not shown).

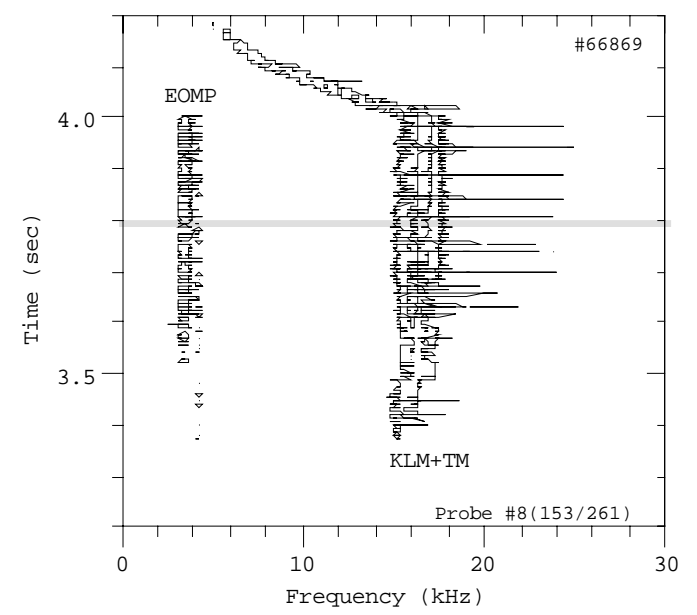

Fig. 24 Contours of equal FFT modulus values of a Mirnov coil signal in shot \#66869 - a standalone EOMP event and a KLM event with a TM companion.

An example contour plot is in Fig. 24 for shot \#66869, a discharge having an EOMP and a KLM with a TM companion at two different frequencies. It used a signal from the Mirnov probe \#8(153/261) sampled at a lower rate of $100 \mathrm{kHz}$ but continuously over a longer period of time in comparison with the usual fast sampled data. FFT's taken over a series of contiguous sub-intervals of the period are the basis of constructing a contour plot. A contour represents a locus of an equal FFT modulus. Contours at only a few levels are in the figure, because its main purpose is to track the time evolution of frequencies of spectral peaks. A gray horizontal line (along the frequency axis) indicates the time period used for analysis of the higher frequency branch (TM) of this discharge in earlier Figs. 10, 14, 18, 22, and 23. It was also the time period used for analysis of the lower frequency branch (EOMP) that appeared in Fig. 6 and 7. A 'mountain ridge' around $4 \mathrm{kHz}$ is the peak that corresponds to the EOMP, and another around $17 \mathrm{kHz}$ is for the TM. This is a typical evolution for discharges with an EOMP and a TM. 
The contour plot in Fig. 25 shows that the EOMP in the 'pure EOMP shot,' \#91560, was a persistent and sole feature nearly throughout the beam heated period (between 2.8 and $4.2 \mathrm{sec}$ - beam power was much reduced over the beginning and ending $0.2 \mathrm{sec}$ sub-periods). Over this extended period the discharge had very large magnetic perturbations without any detectable temperature fluctuations in interior regions. Smaller ridges at approximately twice and three times the frequency are also evident. We will discuss them shortly.

Notice a series of horizontally elongated disjoint contours around $3.1 \mathrm{sec}$. The disjointness signifies 'bursting' oscillations. Elongation means a rapidly changing frequency, or 'whistling,' downward in the present case. These are KLM's with an EOMP companion that slowed down and disappeared quickly before a main stand-alone EOMP emerged.

The EOMP was still robust just before the end of a high power phase of NBI at $4.0 \mathrm{sec}$, but disappeared quickly afterward. These contours lasted some ten milliseconds because of smoothing by a contour plotting algorithm, but coherent oscillations on raw $\dot{B}$ signals vanished much more rapidly in a sub-millisecond time scale. Either the perturbations vanished or stopped rotating. This is a behavior observed in many EOMP discharges.

This contrasts with the evolution of TM's. For example, look at Fig. 24. In the same discharge over the same period (i.e., under precisely the same plasma conditions - except possibly for radial location differences) the EOMP disappeared, but the TM persisted, after NBI termination.

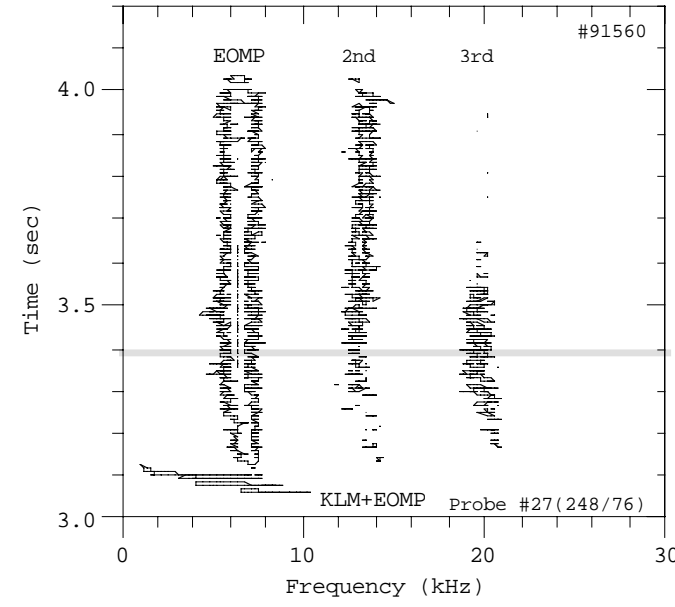

Fig. 25 Contours of equal FFT modulus values of a Mirnov coil signal in shot \#91560 - a pure stand-alone EOMP event.

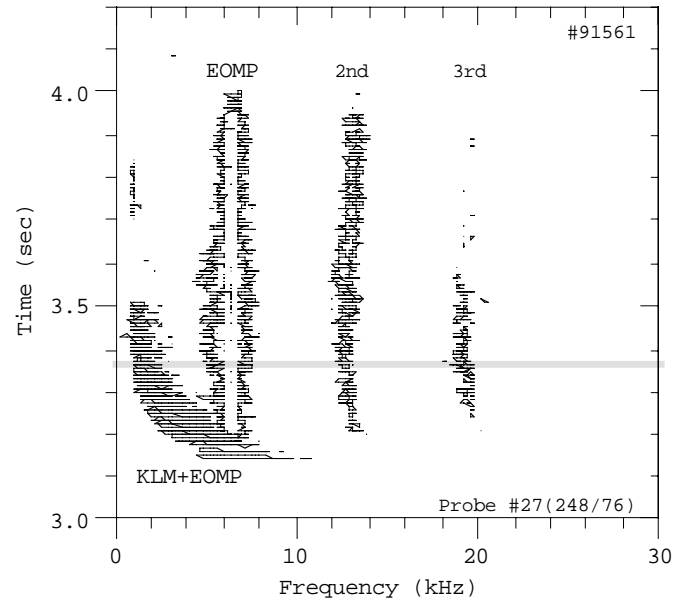

Fig. 26 Contours of equal FFT modulus values of a Mirnov coil signal in shot \#91561 - a KLM event with an EOMP companion $(\# 91561 \mathrm{LF})$ as well as a stand-alone EOMP event (\#91561HF).

The 'KLM/EOMP discharge,' \#91561, evolved slightly differently as evident in Fig. 26. A set of EOMP ridges was present that were similar to those in the previous discharge. But a series of bursting KLM's persisted much longer. This 
discharge thus had a stand-alone EOMP as in the previous shot and a KLM with an EOMP companion at the same time. The latter was the subject of analysis in earlier subsections (Figs. 9, 13, 17 as well as earlier Figs. 6 and 7).

\subsection{Spectral and Spatial Harmonics}

We can extract some information on the spatial (toroidal) structure of EOMP's from the presence of higher spectral (frequency) harmonic ridges evident in Figs. 25 and 26 as well as similar figures for other EOMP discharges.

Observations of higher harmonics in a frequency spectrum often leads to a question as to how they came about: whether a spatially perfect sinusoidal structure was rotating at an uneven speed ('wobbling' or frequency-modulated), or a spatially imperfect sinusoidal structure was rotating at a constant speed. This is an interesting question, because it may yield a general clue about the nature of the perturbations.

Recall that a wobbling rotation speed of a spatially perfect sinusoidal pattern would merely produce a series of sidebands about the central rotation frequency. On the other hand, a spatially imperfect sinusoidal pattern can be expanded into a series of spatial harmonics. They would generate spectral harmonics even when rotating at a constant speed.

Under some special, but not necessarily uncommon, circumstances, a wobbling rotation can generate only upper sidebands at integral multiples of the central frequency. It would happen, if the frequency of rotation modulation equals the rotation frequency itself - rotation gets a 'kick' once every full toroidal turn, for example, as in a locked mode mechanism based on a stationary field error. That would mimic higher spectral harmonics just in the present cases.

But the two possible scenarios have a decisive difference. Upper sidebands generated by a spatially perfect pattern would have to have the same spatial harmonic number as the base harmonic, while spectral harmonics generated by a spatially imperfect pattern would have higher spatial harmonic numbers.

An argument analysis showed that the spatial harmonic number was $n=1$ for the first ridge of the EOMP in shot \#91560 as well as for the first ridge of the stand-alone EOMP in shot \#91561. A similar analysis indicated that the poloidal harmonic number was not reliably determinable for higher order ridges in either shot, because it became comparable to the number of PA probes. But spatial harmonic numbers, $n=2$ for the second ridge and $n=3$ for the third, were reliably determinable for both shots. Thus, the spectral harmonic number was proportional to the spatial harmonic number.

These observations indicate that a spatially imperfect sinusoidal pattern generated magnetic perturbations in these discharges. Robust higher spectral harmonics, as in these discharges, occur in many, but not all, EOMP discharges. 


\subsection{Relationships among Classes}

We have tried to select in this section a discharge that has a single dominant class of perturbations in order to characterize it independently from the others. But a more commonly encountered situation is that more than one class appear together in a discharge over the same period of time and at the same frequency. (The shot \#81178, which appeared in Figs. 6 and 7 and discussed in Sec. 3.4 was such an example.) EOMP's are particularly a ubiquitous presence in many discharges, though dominant in some and merely of a 'parasitic' presence in others.

A large EOMP appears, either alone or together with one or both of the other classes, in almost every discharge with a strong magnetic activity. We often get an impression that the presence of the other classes do not seem to change the intensity of observed magnetic activity much. This suggests that dominant parts of observed magnetic perturbations come from an EOMP, and the other classes contribute relatively little.

We can buttress this impression by a more quantitative assessment by scaling a relationship between the island width and magnetic perturbations using an analytical formula[51] for a cylindrical plasma,

$$
W^{2} \approx 4 r_{s}\left(\frac{B_{r}}{m B_{\theta}}\left|\frac{q}{r_{s} q^{\prime}\left(r_{s}\right)}\right|\right),
$$

where $r_{s}$ is the minor radius of a singular surface, $B_{r}$ and $B_{\theta}$ are the radial and azimuthal field at the surface. The formula states that the perturbation field is proportional to the square of the island width.

For example, let's go back to Fig. 8 where we found an $\mathrm{m} / \mathrm{n}=2 / 1$ island with an average island width of $3.9 \mathrm{~cm}$ and a maximum magnetic perturbation amplitude of $64 \mathrm{mG}$. An $\mathrm{m} / \mathrm{n}=2 / 1$ island twice as large as the one in this discharge would be a robust island, and one three times as large would be among the largest observed in TFTR. They would still correspond to a perturbation amplitude of a quarter to a little over a half Gauss. In comparison a robust magnetic perturbation amplitude is typically several Gauss, and the largest is over ten Gauss. Thus, islands would contribute only a fraction of the observed magnetic perturbations.

This is not necessarily to say that EOMP's do the most damage in a plasma degraded by an MHD activity. It simply says instead that EOMP's 'magnetic activity as measured by Mirnov coils' is dominant. In fact, discharges with only a stand-alone oscillating EOMP do not seem to suffer from its presence in any obvious manner while discharges with an EOMP together with other classes can have a strongly degraded performance. Discharges with other classes only can also result in poor performance. These observations together appear to suggest that, contrary to an initial impression, EOMP's are of no consequence despite their large magnetic perturbations. But it would be premature to reach that conclusion at this point.

We do not yet know many aspects of the EOMP class and its relationships to the 
others. First, how does an EOMP interact with a TM or a KLM? This should be a particularly interesting question, if an EOMP contains a field component that is resonant at a singular surface relevant for an interacting partner. We should consider interactions when they are at the same frequency as well as different frequencies. Second, does an EOMP exchange momentum with another class or with tokamak structures? Third, what does an EOMP do on its own to the plasma when it slows down, or ceases to oscillate? But before letting our imagination run wild, we aim in this article first to establish the existence of EOMP's and to characterize them.

\subsection{Summary of Characteristics of Three Classes}

We summarize characteristics of the three classes of perturbations in TFTR.

- 'Edge Originated Magnetic Perturbations' (EOMP's) have:

1. Toroidal harmonic number unity and poloidal harmonic number comparable to a discharge's edge q-value.

2. Little detectable electron temperature fluctuations, except possibly in edge regions.

3. Rotation in a direction uncorrelated with NBI torque input direction.

4. Poloidal modulus variation patterns that are more general than are expected for MHD modes, including:

(a) Up/down asymmetry.

(b) In/out asymmetry unachievable by a combination of MHD modes that are actually observed internally, and undetected but potentially present.

(c) Outliers in the modulus and argument plots.

5. Toroidally imperfect sinusoidal pattern in some cases.

6. Widespread presence at varying intensities, either alone or with the other classes.

- 'Kink-Like-Modes' (KLM's) have.

1. Little external magnetic perturbations.

2. Internal temperature fluctuations with:

(a) No phase reversal (ideal-mode-like variation).

(b) Localization on a small minor radius side of the $\mathrm{q}=1$ singular surface.

3. A companion, either a TM, an EOMP, or both.

- Tearing-Modes (TM's) examined in this article have.

1. Features generally in line with expectations from MHD modes.

2. External magnetic perturbations with: 
(a) Poloidal modulus variation patterns that are substantially up/down and in/out symmetric.

(b) Total argument variation $2 \mathrm{~m} \pi$ around poloidal circumference.

(c) Rotation in an NBI torque input direction.

3. Internal electron temperature fluctuations with:

(a) Phase reversal (flux surface tearing).

(b) Localization on a small minor radius side of a singular surface compatible with the helicity of external magnetic perturbations.

(c) Magnetic islands generally commensurate in size with external magnetic perturbation amplitude.

\section{MHD-MODE CONTRIBUTIONS WITHIN EOMP}

The EOMP class must contain a source of magnetic perturbations other than MHD modes - a conclusion from two sections earlier. But the class does not exclude MHD modes either. The significance of the EOMP class depends on relative contributions from these two components.

Suppose that an EOMP consisted entirely of an MHD mode, say, a TM, lying hidden in plasma edge regions. It has much significance in practical terms. For example, an RWM control scheme would have to contend with a magnetic 'error' signal that does not obey the feedback loop's causality relationship. It may lead to other consequences of mis-identification. But EOMP's are perhaps less interesting on a more fundamental physics level.

Suppose alternatively that an EOMP came entirely from a source outside the plasma. All issues raised earlier are still there with respect to mis-labeling. But such a source also interacts directly with limiters and walls. It could also interact with MHD modes in interior regions. A possibility of momentum exchange could arise. Such a source could also possess a component that is resonant on a singular surface in interior regions. It could exert a destabilizing force on an MHD mode. EOMP's will become important on a much more fundamental level.

We will undertake in this section a quantitative assessment of MHD mode contributions to EOMP's. We speak mainly of TM's in this context, because they can exist in a radially localized form. EOMP's have no detectable internal fluctuations. But TM's could be present undetected in an EOMP, because they (1) generate temperature changes too small to be recognized as organized fluctuations, (2) 'fall through a crack' between discrete measurement points, and (3) lie outside a GPC viewing domain.

We can approach from two opposite directions in assessing the maximum possible amplitude of TM's that could go undetected. First, we can ask what sorts of poloidal harmonics make up the observed magnetic perturbations. Each of these harmonics is resonant on an integer singular surface. We must be able to see evidence of its 
presence, if it has an amplitude above a detection threshold relevant to its location. In this first approach, we will begin with external magnetic perturbations, and end with internal temperature fluctuations. Second, we can ask whether or not undetectably small TM's, hiding at various locations for one or more reasons listed above, can collectively amount to the observed magnetic perturbations. We will move in an opposite direction in this second approach. But we must first deal with important side issues such as poloidal harmonic content and detection threshold, before we can address the main subject.

The PRIME code is a primary tool for making connections between external magnetic perturbations and internal temperature fluctuations. It uses a onedimensional cylindrical model, and requires concentric flux surfaces and conducting walls. But experimental data reflect a toroidal geometry with shifted flux surfaces and walls. 'Patching' of two disparate geometries will necessitate some approximations.

A 'geometrical correction' method will attempt to correct for the proximity effect, and transfer magnetic perturbations measured on a non-concentric Mirnov circle to a 'virtual wall' that is concentric with a singular surface. Poloidal harmonic expansion will then determine a harmonic content of corrected perturbations.

A 'flux-based' normalized minor radius will patch up the electron temperature measured at major-radial locations to temperatures defined in terms of a 'geometrybased' normalized radius used by the PRIME code. We will convert temperature changes of a given magnitude, here the smallest detectable, to flux surface motion using a measured temperature gradient, and then employ the PRIME code to relate flux surface motion to external magnetic perturbations. This will give a detection threshold.

We made in connection with Fig. 1 a seemingly outlandish proposition that a magnetic signal that looks like a perfect specimen of MHD modes is in fact not what it appears to be. We will substantiate this claim in this section.

Here is what we will say at the end. A part of the observed magnetic signal is definitely an MHD mode. But its contributions are tiny. Another part could be undetectably small MHD modes in interior regions. But its contributions are small. The rest is an EOMP. A part of that EOMP could be an MHD mode hiding in the plasma edge regions. But its contributions fall well short of the total observed magnetic perturbations. We will show that a majority of them originate from something other than MHD modes.

\subsection{Magnetic Perturbations}

\subsubsection{Time and Frequency Domain Signals}

We demonstrate first that magnetic perturbations in Fig. 1 are a 'perfect specimen of MHD modes' to almost any standard when viewed in the time domain. 
Figure 27 presents the time variation of Mirnov signals (integrated once with respect to time) from the discharge. The probe \#8(153/261) and \#15(338/261) signals have a peak-to-peak amplitude of about $0.2 \mathrm{mT}$ and $1 \mathrm{mT}$, respectively. These signals are representative of other probe signals on the outboard and inboard sides, respectively. A higher frequency component appears as small wiggles on the probe \#8 trace, and barely inflexions in the probe \#15 trace in Fig. 27.

A magnetic probe signal in an expanded time scale, such as the probe \#15 trace in this figure, appears to be unmistakably an MHD mode, especially when taken in a context of NBI heating such as the one shown in Fig. 1.

Frequency spectra for these signals (unintegrated) were shown earlier in Figs. 4 and 5. Two prominent peaks at $4.64 \mathrm{kHz}$ and $23.47 \mathrm{kHz}$ in the probe \#8 spectrum had an amplitude, when integrated, of $0.575 \mathrm{G}$ and $0.077 \mathrm{G}$, respectively, a ratio of 7.5. Corresponding peaks in the probe \#15 spectrum had an integrated amplitude of $4.55 \mathrm{G}$ and $0.046 \mathrm{G}$, respectively, a ratio of 98.7. The lower frequency peak was an EOMP, and the higher frequency peak was a KLM with a TM companion. Their spectral evolution was qualitatively similar to the one shown in Fig. 24. The two peaks were persistent features over most of the NBI heating period.

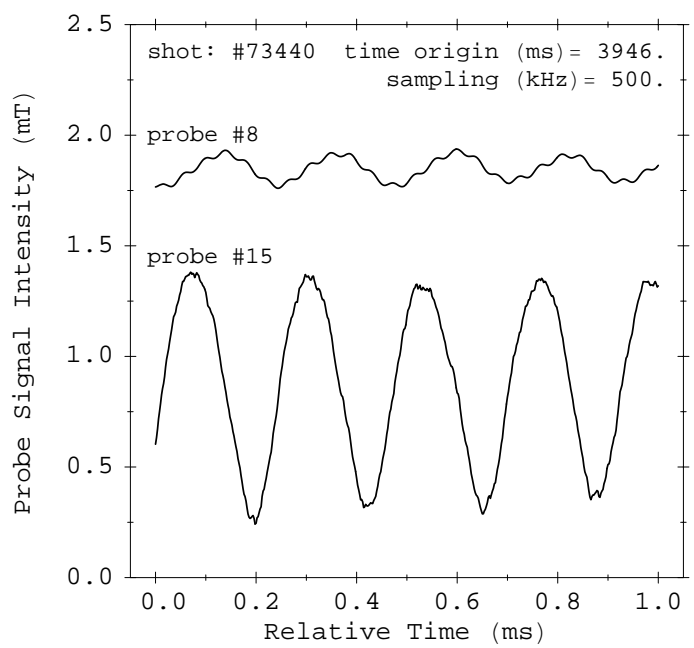

Fig. 27 Integrated magnetic perturbation signals $(B)$ of Mirnov coils \#8(153/261) and \#15(338/261). The signals are displaced vertically by an arbitrary constant amount, and the vertical scale is only for gauging the range of excursion of signals during oscillation cycles. A secular ('DC') trend was removed by subtracting a moving average over a (low frequency) cycle after integration.

\subsubsection{Poloidal Variations}

Figure 28 shows a poloidal variation of the FFT modulus of magnetic perturbations at the lower frequency peak. It exhibits all of the signatures of an EOMP. First, the modulus varied over a wide range. A minimum value of $1.64 \mathrm{~T} / \mathrm{sec}$ was attained by the probe \#8(153/261), and a maximum of $13.4 \mathrm{~T} / \mathrm{sec}$ by the probe \#15(315/261). The max-to-min ratio was 8.4. The in/out asymmetry ratio was 5.9. The down/up asymmetry ratio was 1.4. It had outliers: two NPA probes, \#28(229/16) and \#30(281/9), appear to be out of line from the rest.

A corresponding FFT argument analysis is in Fig. 29. A gray curve is a fit using a Merezhkin corrected angle. The best fit value for the Merezhkin coefficient 
was $\lambda_{M z k}=0.83$. NPA probe data generally aligned well with PA probe data, indicating that the choice, $\mathrm{n}=+1$, was correct for a toroidal harmonic number. Magnetic perturbations thus rotated in a counter-direction opposite to the direction of net torque, +11 Newton-m, applied by NBI. A few data points, including those outliers in the amplitude plot, appear somewhat out of line. But the RMS error was overall only $18 \mathrm{deg}$. The total phase variation was $-10 \pi$, indicating $\mathrm{m}=+5$ for the poloidal harmonic number. It is close to the $q_{e d g e}=5.2$ value, and satisfies another characteristic of an EOMP.

The Mirnov circle is not in general concentric with a singular surface. We will therefore apply geometrical corrections to magnetic perturbations measured on the Mirnov circle, and translate them to equivalent amplitudes on a 'virtual wall.' It is concentric with a singular surface, and has the minor radius of the actual vacuum vessel. The geometrical corrections are based on the $\left(r / a_{s s}\right)^{-(m+1)}$ fall-off of a multipole field in vacuo, where $a_{s s}$ is the radius of a singular surface. They are described in the Appendix B.

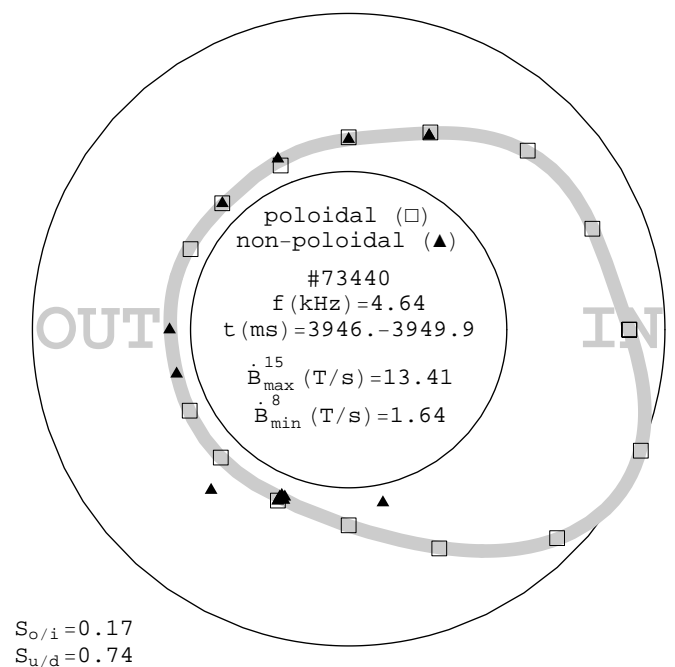

Fig. 28 Poloidal variation of the FFT modulus of Mirnov signals for the low frequency component of shot \#73440.

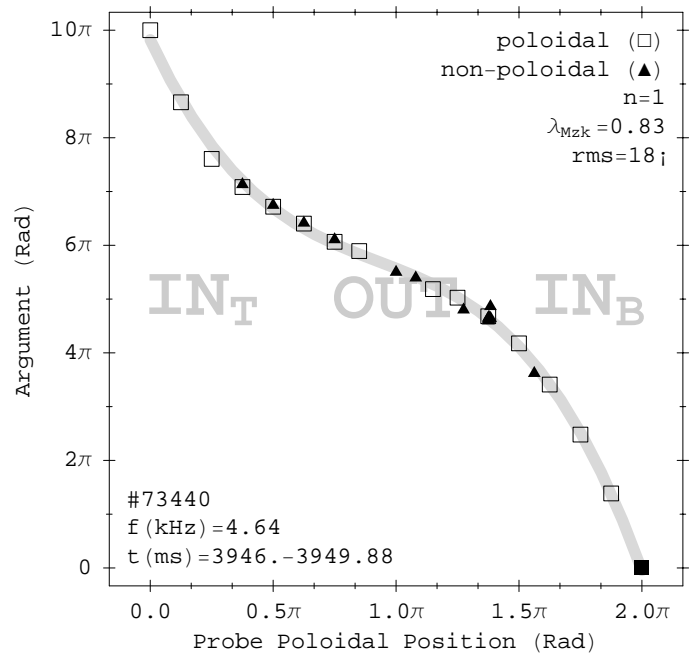

Fig. 29 Poloidal variation of the FFT argument of Mirnov signals for the low frequency component of shot \#73440.

The magnitude of geometrical corrections depends on the poloidal harmonic number through the exponent as well as the radius of a singular surface. We chose $\mathrm{m}=4$ and a corresponding minor radius, anticipating it to be the largest component. The ratio of the virtual wall $(110 \mathrm{~cm})$ to the singular surface $(77.7 \mathrm{~cm})$ radii was 1.42. We have used a Merezhkin coefficient, $\lambda_{M z k}=0.77$, which is a value calculated for the $q=4$ singular surface (Eq. A3). Corrections were not large for this medium bore plasma. Signals on the inboard side became smaller by about $10 \%$ after the corrections, and those on the outboard side increased by a similar amount. The max-to-min ratio changed to 7.2 from 8.4, the in/out asymmetry ratio became 5 from 5.9, the down/up asymmetry ratio remained unchanged at 1.4.

We cannot expand the FFT modulus directly into a poloidal harmonic series 
to obtain harmonic components of magnetic perturbations. This is because the modulus does not have information on relative phases among components. We must perform the expansion instead on the real (or imaginary) part of the FFT. It represents the variation of instantaneous perturbation intensity, and retains the relative phase information. A polar plot of the instantaneous intensity variation in a format similar to the modulus plot is strongly skewed toward the inboard side for the EOMP in this discharge.

We are free to multiply a complex FFT by an arbitrary phase angle to obtain $\dot{B}(\omega) \exp i \theta_{\text {rot }}$, and take the real part of the product. Poloidal harmonic expansion determines the harmonic content at each rotation angle (or equivalently instant of time). The harmonic content is in general not the same at all angles. It can vary strongly for a skewed variation such as the present one.

A Fourier series in a poloidal coordinate system truncated after a finite number of terms is a fitting function. Both cosine and sine terms are present to account for relative phase angles among harmonics. Minimizing of the Root-Mean-Square-Error (RMSE) between values of experimental points and values predicted by a fitting function determines coefficients in the function. The poloidal coordinate here is the poloidal angle corrected for the Merezhkin effect, Eq. A2. The poloidal angle is measured about the center of the $\mathrm{q}=4$ singular surface. This is an approximation as the center varies with the m-number.

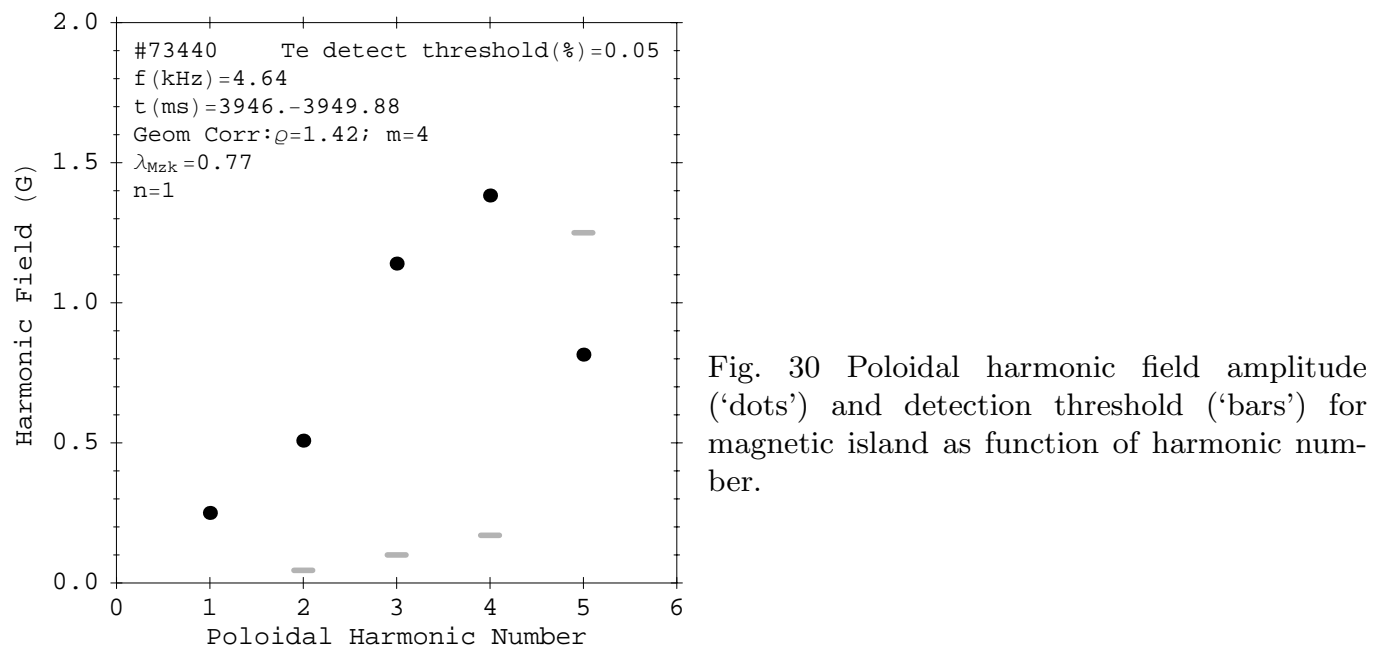

The toroidal harmonic number was unity $(\mathrm{n}=+1)$ from the FFT argument analysis in Fig. 29. Tearing modes with $\mathrm{n}=1$ could occur on integer singular surfaces with harmonic numbers up to $\mathrm{m}=5$ (recall $q_{\text {edge }}=5.2$ ). A fitting function should therefore retain terms up to $\mathrm{m}=5: f(\theta)=c_{0}+\sum_{m=1}^{5}\left(c_{m} \cos \theta^{\star}+s_{m} \sin \theta^{\star}\right)$. The process of fitting to experimental data will determine these coefficients. The process is repeated after incremental rotation of the experimental data. Coefficients values are averaged over a cycle.

The $\mathrm{m}=0$ through 5 harmonic contributions to the absolute values accounted in dimensionless terms for $2,6,12,27,33$, and $20 \%$, respectively of a total field of 
4.2 G. These results are summarized in Fig. 30, and will serve later as a boundary condition for the PRIME code. Notice that the $m=5$ harmonic is the third most important component.

When we use these field components to re-construct a poloidal variation of magnetic perturbations, we see some evidence of spatial aliasing caused by the sparseness of measuring points. It tends to unduly enhance higher-m harmonics at the expense of lower-m harmonics.

\subsection{Electron Temperature Fluctuations}

\subsubsection{Transforming Minor Radius}

A diagnostic usually measures temperature at discrete spatial locations. But a magnetic island would disturb the electron temperature over a region well beyond its own radial extent. The question of whether or not it can detect the presence of an island must therefore take into consideration the proximity of individual measuring points to a relevant singular surface. The PRIME code will determine this 'zone of influence' of an island. But it must first make two-dimensional experimental data conform with its one-dimensional model.

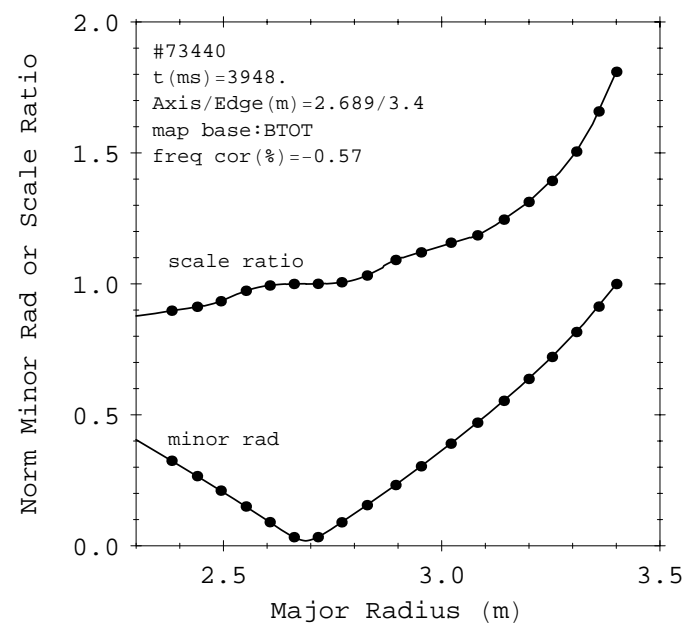

Fig. 31 Flux-based normalized minor radius, and the ratio of scale lengths between the fluxbased minor radius and the major radius, both as a function of the major radius for shot \#73440.

The square-root of the normalized toroidal magnetic flux is a widely used equivalent minor radial coordinate. The TRANSP and SNAP codes both use it. We will call this a 'flux-based normalized minor radius.' The PRIME code uses a cylindrical geometry, and employs another form of normalized minor radius, which is simply the geometrical radius of a circular flux surface divided by the radius of a circular plasma boundary. We will call it a 'geometrical normalized minor radius.' Using these two forms of normalized minor radius interchangeably is our means of establishing a connection between two-dimensional experimental data and onedimensional PRIME code result.

The TRANSP code calculated for the discharge under discussion the minor 
radius shown in Fig. 31 as a function of the major radius in the midplane. The curve in the figure is a spline interpolation to a result calculated at a grid of discrete major radial points (grid points not shown). (It does not reach a zero minor radius at the magnetic axis major radius because of the way the splined result is presented - the minor radius is taken as positive on both sides of the axis.) 'Dots' indicate normalized minor radius values corresponding to major radial points at which GPC1 data were taken. The curve steepens as it approaches the plasma boundary, i.e., a unit distance in the major radius corresponds to a greater interval in the normalized minor radius in edge regions than in central regions.

Figure 31 makes this stretching of scale length more quantitative. It also shows the derivative of the (dimensional) flux-based minor radius with respect to the major radius as a function of the latter. 'Dots' are again the GPC-1 measuring points. The 'minor-radius to major-radius scale length ratio reaches 1.8 at the outboard plasma boundary. Thus, a 'last $5 \mathrm{~cm}$ ' plasma edge region represents a lot more room in terms of the normalized minor radius than the small major radial distance might suggest. A 'larger than imagined' tearing mode could perhaps lay hidden in this space. We will have taken this 'scale length stretching effect' into consideration by making the transformation to the flux-based normalized minor radial coordinate.

\subsubsection{Constructing Temperature Profile}

A profile of electron temperature is in Fig. 32 as a function of the normalized minor radius. 'Dots' on the temperature profile indicate measuring points. Neighboring dots are connected by a straight line segment to construct a profile.

Also shown in the figure is a q-profile obtained in a TRANSP analysis. 'Crosses' on the q-profile are locations of integer singular surfaces between 1 and 5 plus $q=4 / 3,3 / 2$, and $5 / 3$. The singular surface locations are also transferred onto the temperature profile as short vertical line segments. The relative locations of the temperature data points and singular surfaces are evident in the figure.

The above temperature profile was obtained in the following way. The frequency of each GPC channel maps to a major radial location when equated to the local second harmonic electron cyclotron frequency corresponding to the total magnetic field calculated for the location in a TRANSP analysis. The major radius of each measuring point was translated to the normalized minor radius using the information in Fig. 31. The last closed flux surface, computed by a moment expansion code using magnetics data, intersects the outer midplane at $340.0 \mathrm{~cm}$. The TRANSP calculations also used this flux surface geometry as an input. In the above frequency-radius mapping a small correction $(-0.57 \%)$ was introduced into the nominal frequency calibrations of the GPC-1 instrument by requiring that the electron temperatures at a $\mathrm{q}=1$ surface on the inboard $(243.0 \mathrm{~cm})$ and outboard $(293.0 \mathrm{~m})$ sides come out to be the same.

In the present discharge non-thermal emission affected significantly the last data 
point shown in the figure. The data location falls nominally just outside (by $2 \mathrm{~mm}$ ) the outboard plasma edge. But the detector would still collect data from plasma edge regions because of a finite instrumental width (FWHM $3 \mathrm{~cm})$. We reset the temperature at this location to an arbitrary but plausible low value of $50 \mathrm{eV}$ on the physical ground that it pertains to a thin plasma edge layer. The modified data point is shown by a gray dot and a gray line segment in the figure. Non-thermal emission may have affected also the 'second to the last' data point, but to a lesser degree. The data point is left intact in the figure.

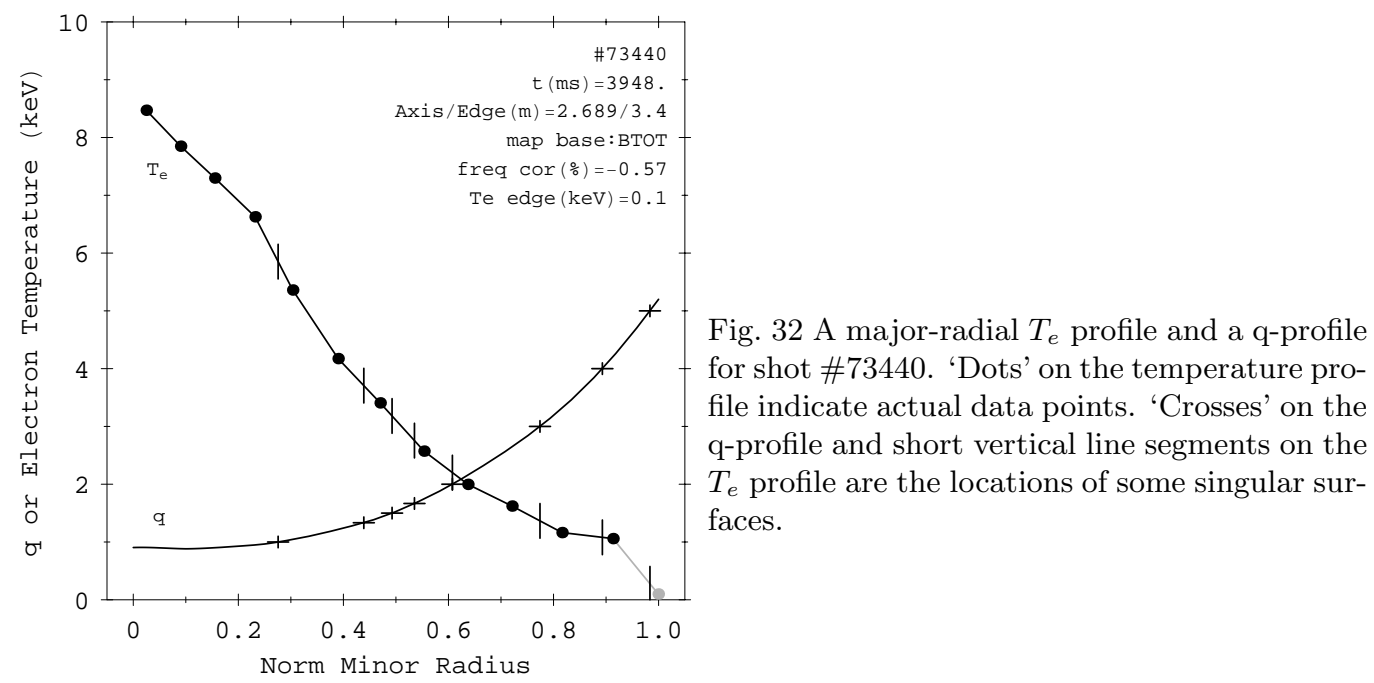

\subsubsection{Temperature Fluctuations}

An EOMP can be huge compared to a TM or a KLM in terms of edge magnetic perturbations. But an EOMP is no match for a TM or a KLM in terms of electron temperature fluctuations in interior regions. These general statements apply to this discharge also.

Temperature fluctuations are shown in a contour plot in Fig. 33. The FFT was taken over a $4.096 \mathrm{~ms}$ window. The frequency grid spacing was thus $244.1 \mathrm{~Hz}$. Gray contours are at 'twice the noise' level or $0.1 \%$. Black contours are for levels from $0.4 \%$ through $1.9 \%$ at $0.3 \%$ steps. The viewing domain boundary was at a major radius, $335.9 \mathrm{~cm}$, and the plasma boundary was at $340 \mathrm{~cm}$. The last $4.1 \mathrm{~cm}$, or about $5 \%$ of the $87 \mathrm{~cm}$ plasma minor radius, was thus not observable. But the unobservable space is nearly $9 \%$ in terms of the flux-based normalized minor radius.

Short horizontal line segments indicate locations of the magnetic axis $(269.7 \mathrm{~cm})$, integer singular surfaces, $\mathrm{q}=1$ through 5 , and a singular surface, $\mathrm{q}=4 / 3$, all calculated in the TRANSP analysis. The $\mathrm{q}=4$ surface is inside the viewing domain boundary by only $1 \mathrm{~cm}$. The $\mathrm{q}=5$ surface is outside the viewing domain, and inside the plasma boundary by only $0.7 \mathrm{~cm}$. Two gray vertical lines, 'b' and 'a,' represent the EOMP $(4.64 \mathrm{kHz})$ and TM $(23.47 \mathrm{kHz})$ frequencies, respectively. A KLM was also present at the TM frequency. 
Black contours appear along the gray line 'a,' indicating the presence of organized activity near the TM frequency. But a central point of this figure presently is the absence of black contours along the gray line 'b.' The EOMP could have a poloidal harmonic component at each of these integer singular surfaces. Black contours would be expected wherever a short line segment (other than magnetic axis line) crosses the gray line. A hint of organized activity exists inside the $q=1$ surface. It was perhaps an incipient KLM (at the EOMP frequency).

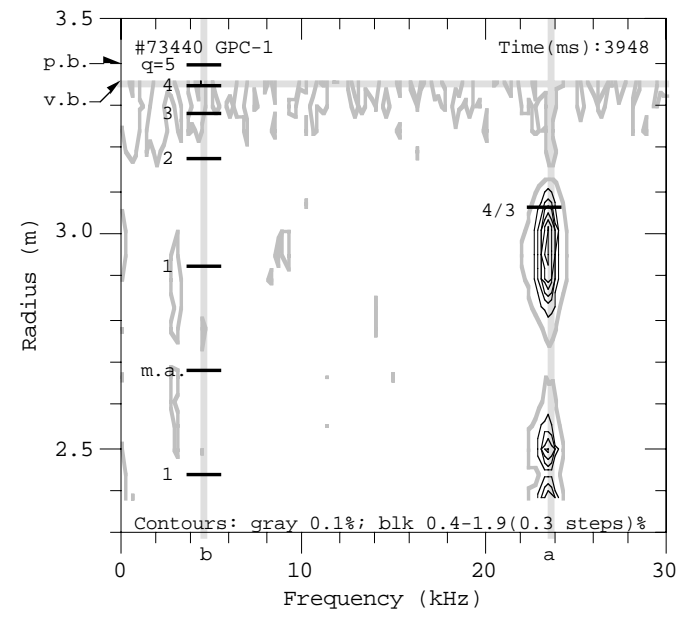

Fig. 33 Contours of equal FFT modulus values of fractional $T_{e}$ perturbations in shot \#73440.

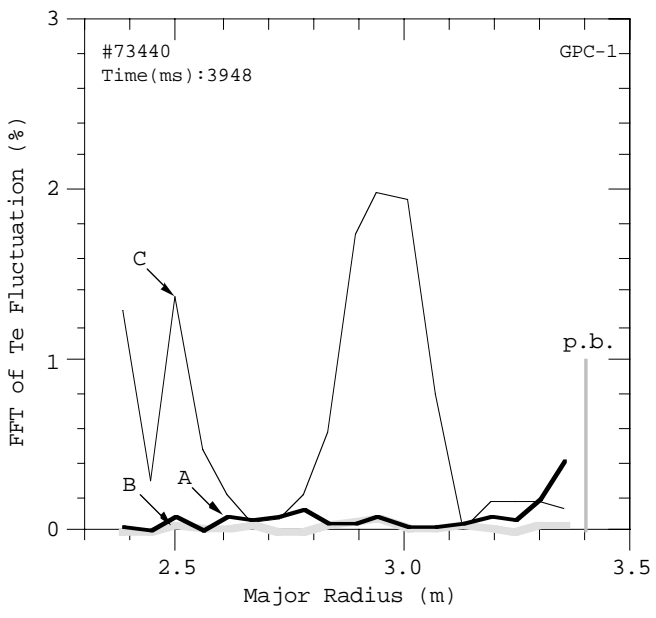

Fig. 34 Radial profiles of fractional $T_{e}$ perturbations at selected frequencies in shot \#73440: $\mathrm{A}(4.52 \mathrm{kHz}), \mathrm{B}(17.9 \mathrm{kHz})$, and $\mathrm{C}(23.6 \mathrm{kHz})$.

Radial 'slices' at specific frequencies of the contour plot will show temperature fluctuations in more detail. Three such radial profiles are in Fig. 34. A thick black curve 'A' $(4.52 \mathrm{kHz})$ is near the EOMP frequency, a thin black curve ' $\mathrm{C}$ ' (23.6 kHz) is near the TM frequency, and a thick gray curve ' $\mathrm{B}$ ' $(17.9 \mathrm{kHz})$ is at an inbetween point in the spectra where fluctuations are at their lowest levels (see Figs. 4 and 5). The curves end on the right hand side at the viewing domain boundary. A gray vertical line segment indicates the plasma boundary.

The curve ' $\mathrm{B}$ ' is at or below the 'twice the noise' level over the entire radial range covered in the figure. The curve ' $\mathrm{A}$ ' is mostly at similar values, rising just above the level over a small interval near the axis. But it rises sharply toward the viewing domain boundary, attaining values well above the level. Note that it is $0.4 \%$ at the last measurement point. The behavior is again suggestive of the presence of larger fluctuations beyond the boundary. The curve ' $\mathrm{C}$ ' reaches a fluctuation level of nearly $2 \%$. It is interesting to recall that the magnetic perturbation field at the wall (in Gauss) was an order of magnitude greater (factors of 7.5 and 98.7 based on probes \#8 and \#15 signals, respectively) for the EOMP than the TM. Yet, temperature fluctuations were smaller by at least a factor of five for the EOMP than the TM. 


\subsubsection{Noise Level}

We want to determine the size of a small island that is about to 'disappear into noise.' We must have a quantitative notion about a noise level in order to accomplish this objective. What is noise in the present context? It is broad-band signals that interfere with our effort to discover a narrow-band organized activity. A relevant noise level is then intimately connected to the way we plan to discover an organized activity.

An organized activity showed up as a set of nested closed contours in the earlier examples. When we reduce the level of signal (normalized FFT modulus) at which contours are drawn to a sufficiently small value, contours, both closed and open, will appear ubiquitously over a plot domain. Some of them may represent an organized activity. But it becomes difficult to identify them with any specific physical phenomenon (islands) we may be looking for. We may then declare that we found a noise level relevant for the task at hand.

Contours in Fig. 35 are drawn at an $0.05 \%$ level. The FFT calculations had the same frequency resolution for this figure and Fig. 33. Ubiquitous contours would make it difficult to be certain about the existence of organized temperature fluctuations in this plot. It thus appears reasonable to take $0.05 \%$ as a relevant noise level. We will call it a 'detection threshold for organized temperature fluctuations.' This is related to, but not yet, a relevant threshold for detecting an island. The latter must take the discrete nature of the GPC measurement into consideration.

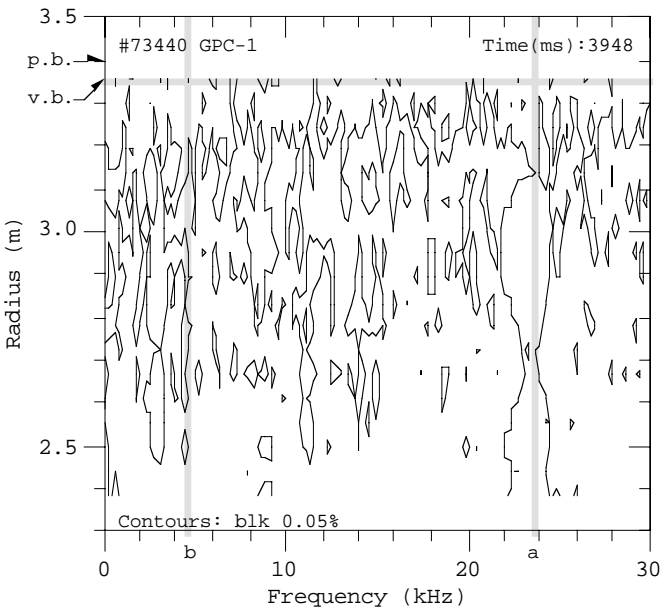

Fig. 35 Contour of FFT modulus at $0.05 \%$ level for shot \#73440.

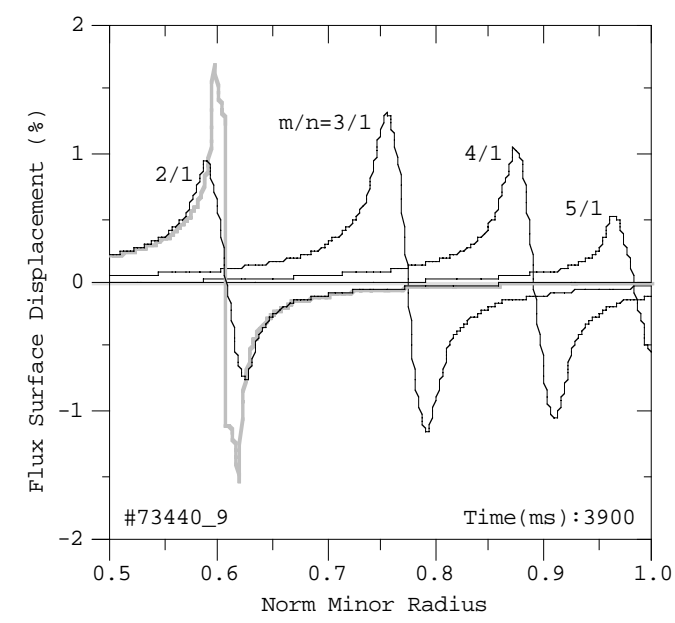

Fig. 36 Displacement of flux surfaces caused by tearing mode islands that are potentially present for the low frequency component in shot \#73440.

\subsubsection{Flux Surface Displacement.}

We want ultimately to make a connection between temperature fluctuations and magnetic perturbations. But we need more immediately to know how much 
an island of a given size (specified in terms of magnetic perturbations) pushes flux surfaces around, because that's what causes temperature fluctuations.

The PRIME code is a key tool in this effort. It uses a poloidal harmonic amplitude on a virtual wall as a boundary condition to calculate a profile of displacement, or 'motion,' of flux surfaces. Figure 30 already presented the harmonic amplitudes for the present EOMP. The PRIME code was run separately for the $\mathrm{m} / \mathrm{n}=2 / 1$ through 5/1 modes. But Fig. 36 reports their flux surface displacement profiles together. Neither $1 / 1$ mode, nor interacting islands, are within the scope of the underlying theory.

The displacement is measured as a percentage of the plasma minor radius. An island displaces flux surfaces from their equilibrium locations radially outward (positive displacement) on one side of it and inward (negative displacement) on the other. 'Bipolar' curves that move into both positive and negative domains in the figure signify this 180 deg phase difference between the two sides. The $2 / 1$ mode has a gray curve and a black curve in the figure to illustrate effect of a finite instrumental width. The gray curve represents a displacement profile as calculated by the PRIME code. The black curve convolves it with a Gaussian sensitivity curve of the instrument with an FWHM width of $3 \mathrm{~cm}$. All other modes present only the convolved curves.

The difference between positive and negative peaks of the gray profile is the calculated normalized island width of the $2 / 1$ mode in the figure. The normalized island widths were $3.0,4.2,3.8$, and $2.7 \%$ for the $2 / 1$ through $5 / 1$ modes, respectively. The instrumental effect reduced these numbers to about 1.4, 2.4, 2.0, and $1.1 \%$, respectively.

Be mindful that we are not suggesting that these islands actually existed. We are instead saying that poloidal harmonic components of the EOMP's magnetic perturbations should have generated this much flux surface motion, if these components actually came from tearing modes. In reality, none of them gave away a clear indication of the existence of an island in a temperature fluctuation contour plot.

\subsubsection{Flux Surface Motion and Temperature Fluctuations.}

We now know how to go from island size (specified in terms of magnetic perturbations) to flux surface motion. Let's work on connecting flux surface motion to temperature fluctuations.

Flux surface motion appears as observable temperature fluctuations, because it occurs in the presence of a temperature gradient. A steep gradient will result in a large temperature excursion for a given displacement. The following expression describes such a relationship.

$$
d_{f}(\rho) \frac{d \mathcal{T}_{e}(\rho)}{d \rho}=2 \tilde{\mathcal{T}}_{e}(\rho),
$$


where $\rho$ is the normalized minor radius coordinate, $d_{f}(\rho)$ is flux surface displacement, $\mathcal{T}_{e}(\rho)$ and $\tilde{\mathcal{T}}_{e}(\rho)$ are time-averaged and fluctuating components of 'true' electron temperature, respectively, which agrees with the measured temperature, $T_{e}(\rho)$, at measuring points, but is hypothetically known continuously as a function of $\rho$. The factor, 2, in the formula takes into account that displacement refers to a peakto-peak excursion while Fourier analyzed temperature fluctuations refer to a peak amplitude. The formula left out the frequency dependence for simplicity.

Let $g_{i}(\rho)$ to be a Gaussian response function normalized to unity, i.e., $\int_{-\infty}^{+\infty} d \rho g_{i}(\rho)=$ 1 , for $\mathrm{i}=1 \ldots N_{c}$, where $N_{c}=20$ is the number of channels in the GPC instrument. Multiply both sides of Eq. 3 by an i-the response function. Integrating the result leads to another form.

$$
\int_{-\infty}^{+\infty} d \rho g_{i}\left(\rho-\rho_{i}\right) d_{f}(\rho) \frac{d \mathcal{T}_{e}(\rho)}{d \rho}=2 \int_{-\infty}^{+\infty} d \rho g_{i}\left(\rho-\rho_{i}\right) \tilde{\mathcal{T}}_{e}(\rho)
$$

The integral on the right-hand-side is a fluctuating component of electron temperature, $\tilde{T}_{e}(\rho)$, actually measured at the i-th measuring point, $\rho=\rho_{i}$, i.e., with instrumental effect folded in. Assume that $d \mathcal{T}_{e}(\rho) / d \rho$ varies slowly compared to $g_{i}(\rho)$. Take the former out from under the integral sign. Approximate it by a temperature gradient at an i-th measuring point. Arranging terms in dimensionless groupings leads to the following expression.

$$
\int_{-\infty}^{+\infty} d \rho g_{i}\left(\rho-\rho_{i}\right)\left(\frac{d_{f}(\rho)}{a_{p}}\right) \approx 2\left(\frac{L_{e}\left(\rho_{i}\right)}{a_{p}}\right)\left(\frac{\tilde{T}_{e}\left(\rho_{i}\right)}{T_{e}\left(\rho_{i}\right)}\right),
$$

where $L_{e}(\rho) \equiv T_{e}(\rho) /\left(d T_{e}(\rho) / d \rho\right)$ is an electron temperature gradient scale length.

The right-hand-side of this relationship is evaluated at an i-th measuring point, and yields a single numerical value. The PRIME code can carry out the integration on the left-hand-side to obtain another numerical value, given as a boundary condition the size of an island in terms of magnetic perturbations. An iterative procedure to match the two values at an i-th measuring point could be a mechanism to determine the size of an island that produces temperature fluctuations observed at the measuring point.

An analysis of a noise level led earlier to a 'detection threshold for organized temperature fluctuations.' Its value was $5 \times 10^{-4}$ for this shot. Substitute this threshold value for $\tilde{T}_{e}\left(\rho_{i}\right) / T_{e}\left(\rho_{i}\right)$ in Eq. 5 for each of the GPC-1 measuring points. The temperature gradient is obtained from Fig. 32. The average of gradients to the left and right of a measuring point is taken as the gradient at the point. This is a well-defined quantity, because straight line segments connect the measuring points, and the gradient is thus constant between them.

Figure 37 shows a resulting 'detection threshold for flux surface motion.' We will also call it a 'detection threshold for an island.' 'Dots' indicate the measuring points. Only a relatively large flux surface motion is detectable in central regions where the temperature profile is nearly flat. But a small motion gets noticed in edge 
regions with a steep temperature gradient. The threshold is relatively constant in inbetween regions.

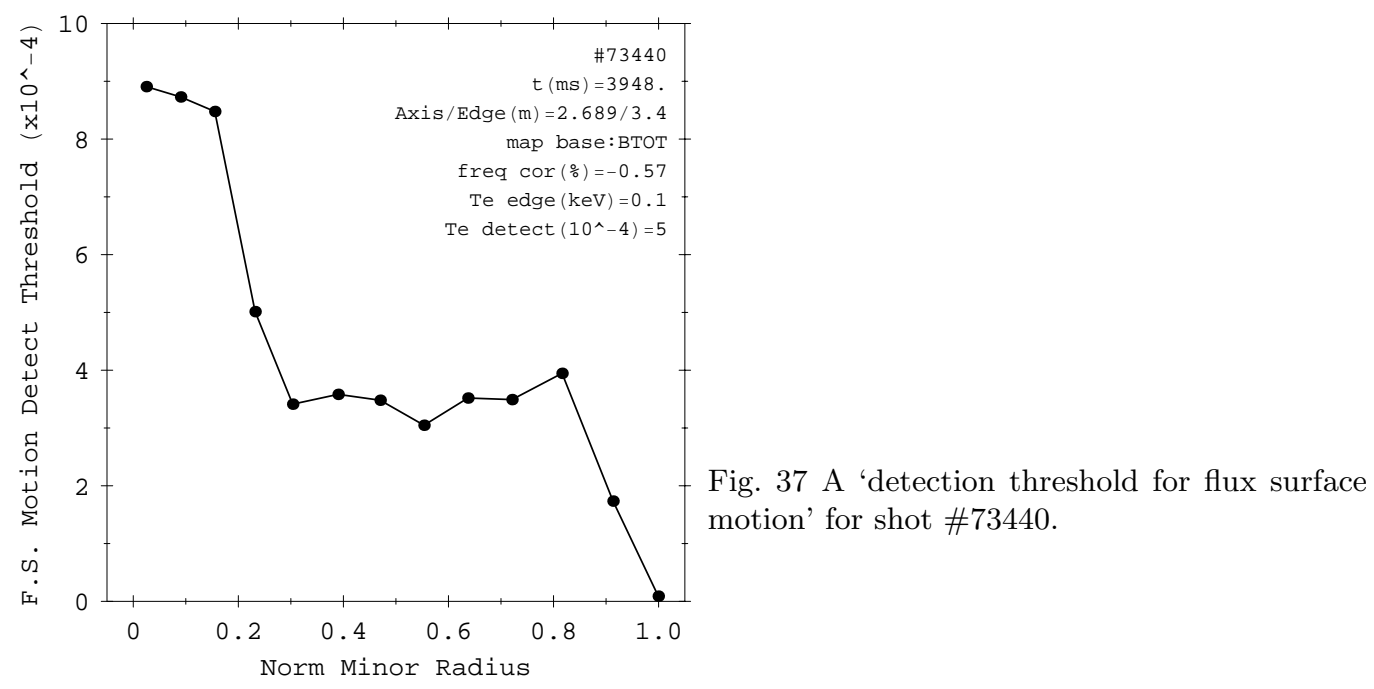

\subsubsection{Hidden Islands}

We will use the tool just developed to find out how big an island can 'fall through a crack,' and escape detection.

The left-hand-side of Eq. 5 yields a finite number of numerical values for a set of discrete measuring points. By letting this number become large, or treating $\rho_{i}$ as a continuous variable, it produces a continuous curve. Such a curve calculated by the PRIME code is in Fig. 38 for a 3/1 mode. A set of 'dots' above zero (horizontal axis) in the figure shows a relevant part of the 'detection threshold for flux surface motion' transferred from Fig. 37. The set is also 'mirrored' with respect to zero to signify that the threshold applies to both positive and negative displacement.

Consider an iterative process of repeatedly calculating a curve similar to the one in Fig. 38, beginning from a small enough perturbation strength that an entire curve fits between the two rows of dots, and then increasing the strength in each iteration. The process stops when a curve 'crosses' a dot. A curve for a different mode may in general cross a different dot. The curve in the figure for a $3 / 1$ mode has just crossed a dot shown in black. A curve for an $2 / 1$ mode would cross a second dot from the left. A curve for a 4/1 or 5/1 mode would cross the last dot from the right.

Recall that the last dot from the right was of a different nature than the rest. It came from small but observable organized temperature fluctuations near the viewing domain boundary. The other dots came from a noise threshold. Interpretation of a mode amplitude found at the process's end may accordingly be different. A value found for a $2 / 1$ or $3 / 1$ mode is an upper bound to the size of an island, if one existed, though no signs of its presence were evident in temperature fluctuations. 
An amplitude found for a $4 / 1$ or $5 / 1$ mode is an island size corresponding to the observed temperature fluctuations. The difference in interpretation is subtle, and may not warrant any further reference.

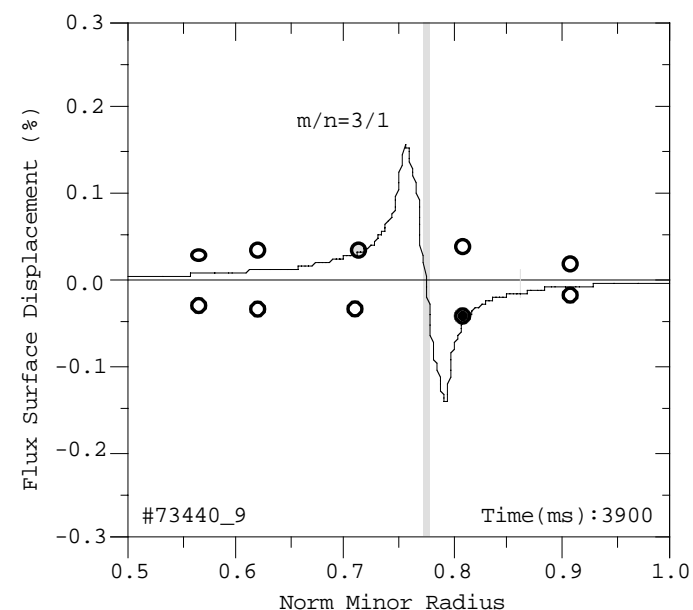

Fig. 38 Shows an island that has grown just large enough to be detected. The example is a $3 / 1$ mode island.

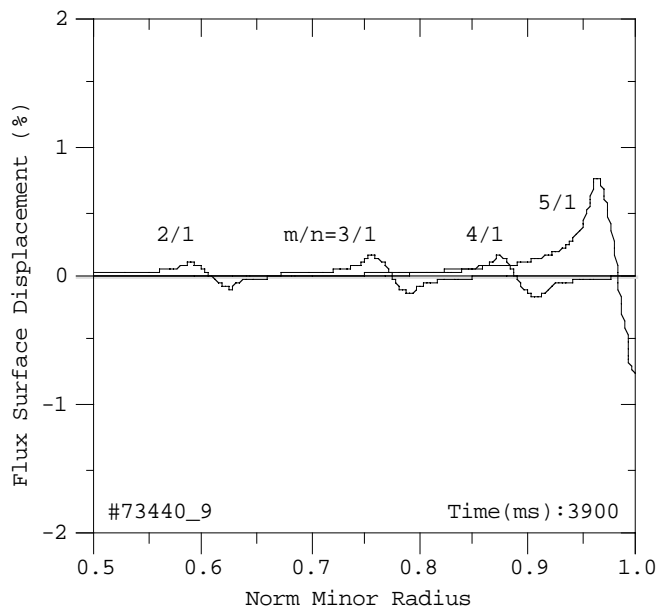

Fig. 39 Shows an upper bound of tearing mode island size that is consistent with temperature fluctuations, either actually observed (for $\mathrm{m} / \mathrm{n}=4 / 1$ and $5 / 1$ ) or at the detection threshold (for $\mathrm{m} / \mathrm{n}=2 / 1$ and $3 / 1$ ).

But one thing is worth mentioning. The latter interpretation assumed the existence of an 'unseen' island. It produced both magnetic perturbations observed outside the plasma and temperature fluctuations observed within the viewing domain. Another interpretation is possible. Some magnetic perturbations outside the plasma of an unspecified origin caused temperature fluctuations within the viewing domain; skip the island altogether. This opens up an interesting possibility in interpreting the nature of EOMP's - a subject of the discussion section.

'Detection thresholds for an island' determined in the procedure were 0.045, $0.1,0.165$, and $1.25 \mathrm{G}$ for $2 / 1,3 / 1,4 / 1$, and 5/1 modes, respectively. Figure 39 assembles together displacement profiles at these threshold values. The figure has the same axes limits as Fig. 36 for an easy visual comparison.

We have completed a connection between magnetic perturbations and temperature fluctuations. First, we began from harmonic components of measured magnetic perturbations, and transformed them into expected flux surface displacement shown in Fig. 36. Second, we started from temperature fluctuations, either thresholds or measured values, and converted them into flux surface displacement shown in Fig. 39. The two halves of the long process are also summarized in Fig. 30 in terms of magnetic perturbations; Short horizontal line segments, or 'bars,' indicating the threshold values are compared with the dots showing the observed values.

A largest possible 2/1 island, if existed, that could have been buried in noise in temperature fluctuations represented only $0.045 \mathrm{G} / 0.51 \mathrm{G}=8.8 \%$ of a relevant harmonic of the observed magnetic perturbations. A corresponding number for 
a $3 / 1$ island was also $0.1 \mathrm{G} / 1.14 \mathrm{G}=8.8 \%$. An 'unseen' 4/1 island that could have produced temperature fluctuations observed at the last measuring point was $0.165 \mathrm{G} / 1.38 \mathrm{G}=12 \%$ of the magnetic perturbations. On the other hand, an 'unseen' $5 / 1$ island that could have produced the same temperature fluctuations was $1.25 \mathrm{G}$. It was greater than a relevant harmonic component $(0.82 \mathrm{G})$ of the magnetic perturbations. In other words, the whole $5 / 1$ harmonic could have come from a hidden island.

\subsubsection{Allowance for Errors}

Locations of measuring points and singular surfaces are clearly important in assessing an upper bound to the island size. We need to make an allowance for errors in the relative locations. Uncertainties arise primarily from a forced marriage of two different geometries, and also from q-profile calculations to a much lesser extent. We may note that locations of singular surfaces in plasma edge regions are rather insensitive to parameter variations, because the profile has a steep radial gradient there, and is also constrained at the close-by plasma boundary.

Return to Fig. 38. This illustrates a threshold assessment that happens to be robust against errors in the relative position of a curve with respect to dots. Imagine the curve in the figure moving to the left. The black dot would no longer be a limiting point on the outboard side. But the curve then crosses another dot shown in gray on the inboard side. This happens without a change in the amplitude, and leaves the assessment unchanged. In other words, the curve as drawn already represents a biggest island that could 'fall through a crack.' Note that the curve at its peak is nearly four times as high as the level of dots. An island can go undetected that is perhaps surprisingly large in comparison with a level of displacement corresponding to a noise threshold in temperature fluctuations. The threshold determined for the $2 / 1$ island was also a robust value.

The 4/1 island case was different. The last dot from the right limited its profile on the outboard side at a 'steep' point. And the second dot from the right was far from limiting the profile on the inboard side. We will give arbitrarily a factor of three allowance to the threshold value determined in the analysis for this island. A change in the relative location of \pm 0.02 in terms of the normalized radius would still keep the assessment valid.

It is somewhat moot to find a similar allowance in the assessment for the 5/1 island, because it is already above the observed magnetic perturbations. If the last measuring point moves by 0.02 in terms of the normalized radius away from the $\mathrm{q}=5$ surface, the assessment would increase to $1.8 \mathrm{G}$ from $1.25 \mathrm{G}$. If the measuring point moves in an opposite direction, it would decrease to $0.8 \mathrm{G}$.

A displacement curve crosses zero at approximately the midpoint of an island. When a measuring point falls right at that point, it registers no temperature fluctuations in spite of the island's presence. But the curve's slope is very steep there. 
A slight offset from the midpoint will push the measuring point out of the 'dead zone,' and bring it above a noise detection threshold. The 'dead zone' is a gray vertical strip in Fig. 38. It is about $0.6 \%$ in normalized minor radius, and $4 \mathrm{~mm}$ in the dimensional major radius, in the case of $3 / 1$ island shown in the figure.

The element of time should be brought into consideration here. How long could a measuring point remain in a 'dead zone?' A q-profile will evolve over time, and change the singular surface locations. Measuring points could also move as the total magnetic field ('mod B') evolves. While a coincidence can happen momentarily, we think it highly unlikely that a large island can hide from view through this mechanism over the life time of an EOMP.

\subsubsection{Summing Contributions}

We began this section asking how much of the observed magnetic perturbations shown in Fig. 1 is attributable to MHD modes. We are now in a position to assess it.

The higher frequency component was for the most part a TM and a KLM, i.e., MHD modes, and had $0.084 \mathrm{G}$ on the virtual wall. The lower frequency component was for the most part an EOMP, and had $4.16 \mathrm{G}$ on the virtual wall. This makes $2 \%$ of the total field (in terms of Gauss) positively attributable to MHD modes.

How much is potentially attributable to MHD modes? Parts of the EOMP could have been MHD modes. The detection thresholds for the $2 / 1$ and $3 / 1$ poloidal harmonics were $0.045 \mathrm{G}$ and $0.1 \mathrm{G}$, or 1.1 and $2.4 \%$, respectively, of the EOMP field. The organized temperature fluctuations observed near the viewing boundary could have originated from a $4 / 1$ or $5 / 1 \mathrm{MHD}$ mode. If the fluctuations were ascribed to a $4 / 1$ mode, it would have had $0.5 \mathrm{G}$ (three times $0.165 \mathrm{G}$ including error allowance), or $12 \%$, of the EOMP field. If they were attributed to a $5 / 1$ mode, it would have had $0.82 \mathrm{G}$, or $19.7 \%$. We will take the greater of the two, but not both, because they attempt to explain the same observed fluctuations. The $1 / 1$ harmonic could not have been evaluated, because the theory underlying the PRIME code did not apply to it. It had $0.25 \mathrm{G}$, or $6 \%$. (The $0 / 1$ harmonic was $0.068 \mathrm{G}$, or $1.6 \%$. Its origin was obscure. But we do not think it was an MHD mode.) All told, 29.2\% of the EOMP field could have been MHD modes.

Adding up $2 \%$ of the total field and $29.2 \%$ of the EOMP field leads to $30.6 \%$ of the total magnetic perturbations (in terms of Gauss). This much was, or could have been, attributable to MHD modes. (A similar assessment could be done in terms of $\mathrm{T} / \mathrm{sec}$. It increases the TM and KLM contributions by a factor of five to 10\%. The total will reach $38.6 \%$.)

We will round the numbers. A couple of percent of the magnetic perturbations in Fig. 1 were positively identifiable with MHD modes. A third could have been MHD modes. 
We have been focusing on MHD mode contributions. But we are ultimately more interested in what comes from sources other than MHD modes. Two thirds of what appeared to be a perfect specimen of MHD modes were not MHD modes according to this analysis.

\subsection{Section Summary}

We examined a typical EOMP discharge to assess contributions to its magnetic perturbations originating from sources other than MHD modes.

The discharge had both a stand-alone EOMP and a TM (along with a small KLM), but at two different frequencies. We evaluated contributions from the explicitly observed TM and those TM's that could potentially have existed in plasma edge regions as a part of the EOMP, though never actually been observed.

We summarize result of the examination:

1. A third of magnetic perturbations could potentially have come from TM's that are undetectable, because they either fit between discrete measuring points of a GPC instrument or lie in plasma edge regions beyond its viewing domain.

2. At least two thirds, but possibly nearly all, of magnetic perturbations originated from sources other than MHD modes.

3. EOMP's are potentially important on a fundamental level to physics of low frequency MHD modes in TFTR, including LM's, NTM's, and RWM's.

\section{CONCLUSION}

We examined coherent perturbations in TFTR at low frequencies, typically at several $\mathrm{kHz}$, but ranging up to $30 \mathrm{kHz}$, and found the following.

1. Magnetic perturbations observed in TFTR often do not conform to expectations from MHD modes.

2. The observations are consistent with a model consisting of three classes of magnetic perturbations:

(a) Edge Originated Magnetic Perturbations (EOMP's).

(b) Tearing Modes (TM's).

(c) Kink-Like Modes (KLM's).

3. EOMP's are primarily responsible for discrepancies between observations and expectations.

4. EOMP's have: 
(a) Toroidal harmonic number unity and poloidal harmonic number comparable to a discharge's edge q-value.

(b) Little detectable electron temperature fluctuations, except possibly in edge regions.

(c) Rotation in a direction uncorrelated with NBI torque input direction.

(d) Poloidal modulus variation patterns that are more general than are expected for MHD modes, including:

i. Up/down asymmetry.

ii. In/out asymmetry unachievable by a combination of MHD modes that are actually observed internally, and undetected but potentially present.

iii. 'Outliers' (data points that do not fit in the poloidal variation trend) in the modulus and argument plots.

5. EOMP's must include a source of magnetic perturbations other than MHD modes; EOMP's can include MHD modes in plasma edge regions.

6. At least two thirds, but possibly nearly all, of magnetic perturbations in a typical EOMP originate from a source other than MHD modes.

7. TM's examined in this work have features generally in line with expectations from MHD modes.

8. KLM's have little external magnetic perturbations.

9. KLM's have internal temperature fluctuations with:

(a) No phase reversal (ideal-mode-like variation).

(b) Localization on a small minor radius side of the $q=1$ singular surface.

10. A KLM appears always accompanied by a TM, an EOMP, or both.

\section{DISCUSSION}

We have drawn conclusions for our investigation based on experimental observations and reasonable inductive inference through their analysis. We offer here a discussion based on varying degrees of conjecture. Such a discussion may be useful in expounding more freely on implications of the conclusion, stimulating new ideas, and indicating a direction of future effort. But should some speculative thinking prove wrong, it should have no direct consequences on the conclusions already drawn. 


\subsection{Implications for MHD Modes}

An EOMP, or more precisely that part of an EOMP that is not an MHD mode, has implications for tokamak research on two different levels. These are potential consequences regardless of what the source of an EOMP is. On a superficial level it is mis-identification - a matter of calling magnetic perturbations an MHD mode when they in fact are not, though its consequences could still be important in many practical ways. On a fundamental level, if an EOMP, generated by current flowing somewhere outside the plasma, has a field component resonant on a singular surface inside the plasma, it has more profound implications for MHD phenomena.

\subsection{1. $\quad$ Locked Mode Studies}

A traditional model for locked modes ascribes slowing down of a rotating tearing mode to the image current it creates in tokamak structures, and cessation of rotation to a structural field error. The model also attributes detrimental effects on the plasma performance, including disruptions, observed after mode 'locking' to the growth of magnetic islands caused by a component of structural error field that is resonant on a relevant singular surface. The resonant condition occurs only after mode rotation ceases as the structural error field is stationary.

Conventional locked mode thinking has difficulty in explaining some aspects of experimental observations, at least in TFTR. The proposed slow-down mechanism, image current, is always present whenever a rotating MHD mode exists. The proposed locking mechanism, a structural field error, is a permanent feature of a tokamak. But we observe experimentally some magnetic perturbations slowing down and locking in a discharge, and then encounter similar magnetic perturbations not slowing down or locking in a similar discharge. The conventional model appears to lack mechanisms that can come and go depending upon discharge conditions.

An EOMP could have an impact on locked mode thinking on two fronts, one as a provider of a mode slow-down and locking mechanism, and the other as a provider of a mode growth mechanism.

It could serve as a 'field error' with which a tearing mode exchanges momentum. Current that generates an EOMP flows outside the plasma, and therefore interacts with the tokamak structure, in turn could transfer momentum to it. An EOMP plays in this thinking the role of an intermediary 'agent' for brokering momentum between tearing mode and tokamak structure.

A large EOMP is typically several Gauss, but can reach over ten Gauss in some circumstances. Conventional thinking has viewed a structural field error as small as a fraction of a Gauss as significant. An EOMP could be a 'dynamic' field error that comes and goes depending upon discharge conditions.

A component of an EOMP can be resonant on a singular surface at a finite frequency. An EOMP and a tearing mode could lock on to each other, either while 
rotating or stationary. It appears that, in the presence of an EOMP, whatever destabilizing mechanisms invoked in the traditional locked mode model should be operative to spur the mode growth even without slow down or cessation of mode rotation. In other words, an EOMP could affect tearing modes in a much more general way.

\subsubsection{Neoclassical Tearing Mode Studies}

A classical theoretical stability criterion for tearing modes is based on the resistive stability parameter[52], $\Delta^{\prime}$, a quantity that characterizes free energy stored in the confining magnetic field. Disagreement between this theoretical prediction and experimental observations under some circumstances led to introduction of other de-stabilizing influences.

Among them is neoclassical current driven in part by the temperature gradient. The neoclassical theory predicts that flattening of the temperature gradient inside a magnetic island produces a deficit in neoclassical current at 'o'-points in relation to ' $\mathrm{x}$ '-points of the island, and contributes to de-stabilizing a classically stable tearing mode. Neoclassical current influences the stability through the resonant magnetic perturbation field that it generates on a resonant singular surface.

It is again a matter of resonant field. Could an EOMP play a role here - a resonant field originating from outside the plasma, rather than from inside an island?

Of course, we have not yet said anything about the origin of an EOMP, or shown that it indeed possesses a resonant field. These will be a subject of future research.

\subsubsection{Resistive Wall Mode Studies}

An RWM is a variant of the external kink mode for which the finite conductivity of the surrounding walls modifies their stabilizing effect. A theoretical analysis shows that, should a kink mode become destabilized, it is likely to leave its marks on temperature fluctuations deep inside the plasma, because a high pressure gradient is an important driving mechanism, and couples external and internal modes.

An EOMP is commonplace in TFTR. Should it also occur in a tokamak with feedback control of an RWM, it could present a challenge. A mechanism to discriminate between their contributions to measured magnetic signals may become necessary for the feedback mechanism to succeed, because they obey different cause and effect relationships.

\subsection{Current in SOL Plasma}

We propose current flowing in the Scrape-Off-Layer (SOL) plasma as a candidate for a source of magnetic perturbations that can mimic MHD modes. We imagine 
that it flows in an SOL plasma along field lines, strikes a limiter at some point, enters the vacuum vessel walls, and goes out through a limiter at another point to close its circuit.

Field generated by such current can have a poloidal variation that is more general than is allowed for an MHD mode. For example, it does not have to be up/down symmetric, or the same in every discharge. Current may be at a varying distance from Mirnov coils while it is in the SOL plasma. But it is always close to some Mirnov coils while in vessel walls, because a Mirnov circle is right next to them. Current, even spread thin, may still make a significant contribution to Mirnov signals. It could also influence them locally and selectively to make them outliers. Current may not completely encircle the plasma poloidally, depending upon where

it strikes limiters. A part of the poloidal circumference, for example, an inboard part near midplane, could be missing from the current path.

The model is far from being concrete. We cannot presently offer a prescription, other than a general outline given above, of an actual current path corresponding to specific observed magnetic perturbations.

We do not know a physical mechanism that drives such current either. This is clearly an important physics issue. But it is just as important to think about consequences of this current - we offered some of our thoughts above.

We may be able to eliminate the current, even though we do not understand its driving mechanism, by cutting off its path, or greatly increasing its circuit resistance. Such methods may turn out to be much simpler than many schemes that have been attempted or planned.

We do actually detect such current in another tokamak that correlates with various low frequency magnetic phenomena (see below).

\subsubsection{Our Earlier Works}

We have advanced the proposition on SOL current in our earlier works $[17,54-58]$ on Stationary Magnetic Perturbations (SMP's) in TFTR. We believe that they are the same phenomenon as locked modes in other tokamaks. But we did not use the latter term for TFTR, because we did not think modes are central to the observed phenomenon. Difficulties in understanding SMP's observed in TFTR in terms of a traditionally accepted explanation for locked modes motivated us in advancing the proposition.

In the first instance, we saw $[17,55]$ disappearance of temperature fluctuations associated with an MHD mode after it ceased to rotate while magnetic perturbations persisted at a finite level. This suggested that SMP's and MHD modes are separate magnetic phenomena.

In a second instance, we observed[56] the powerful effect of an SMP on transport in central plasma regions as soon as it appeared. We made these observations in 
connection with the disappearance of sawtooth crashes during an SMP. An MHD mode did appear subsequently in the process, but only after the SMP's effect on the transport became evident. This again suggested that SMP's and MHD modes are separate magnetic phenomena.

In a third instance, we recognized[57] a high correlation between occurrence of SMP's and what has become known as 'carbon blooms.' The latter causes massive injection of carbon and other impurities into the plasma followed by a severe degradation of plasma performance. Carbon blooms had been thought of as a purely atomic physics phenomenon between the SOL plasma and limiters. Concurrence of SMP's with blooms suggested something akin to arcing at limiter surfaces.

We called[17,55] the postulated current 'halo current.' Our future plan calls for expanding on each of these observations.

We attempted to understand SMP's through careful comparisons of discharges having an SMP - with its characteristic slowing magnetic oscillations - against discharges having more 'normal' MHD modes - with oscillations that did not slow down. We could discern little differences between the two types of discharges. Our 'normal' MHD modes turned out to involve an EOMP, as most large amplitude magnetic perturbations in TFTR did, and were anything but 'normal.'

\subsubsection{Our Present and Future Works}

TFTR lacked a diagnostic to measure current flowing in and out of its limiters. An effort to detect SOL current that mimics MHD modes has been under way in the DIII-D tokamak[61] instead. The experiment utilizes its Tile Current Array (TCA) diagnostic[64] to measure current in and out of selected tiles in its divertors. We have already observed[59,60] current that oscillates at, or near, the frequency of a concurrently present MHD mode, though not as frequently as we might have expected from our TFTR experience. We have also observed[60] enhanced toroidally non-axisymmetric tile current during the period of low frequency magnetic phenomena, including LM's, RWM's, and ELM's. This is a work in progress.

\subsubsection{Historical Perspective}

A notion that extraneous current flows in a tokamak, other than the one intended to sustain a magnetic configuration of the device, goes back many decades. It includes studies on unipolar arcs in tokamaks in the nineteen seventies[62]. Even concurrent observations of unipolar arcs and tearing modes have been reported[63].

Current after a disruption, flowing along open field lines in vacuum, striking and entering a tokamak structural element at some point, running through vacuum vessel walls, and re-emerging at a structural element at another point to complete its circuit, could exert a large force on the structure. Such current was thought as a major threat to the structural integrity of a future tokamak reactor. The current was 
called the 'halo current.' Considerable experimental effort[64-66] has been mounted to measure its degree of axisymmetry (or lack thereof). Our conjecture on SOL current as an origin of EOMP's is a direct extension of the post-disruptive halo current to the normal discharge period.

\section{ACKNOWLEGEMENT}

The authors would like to acknowledge gratefully their indebtedness to many colleagues for assistance in the course of carrying out their research work described in this article.

The authors are grateful to Dr. G. Taylor for providing them the Electron Cyclotron Emission (ECE) data, and for suggestions for improving the manuscript.

The authors thank Drs. R. Budny, D. Ernst, and B. LeBlanc for TRANSP

code calculations, Dr. D. Ernst for assistance in SNAP code calculations, and Dr. J. Manickam for PEST code calculations. The authors thank Drs. R. Bell, B. LeBlanc, and E. Synakowski for Charge Exchange Recombination Spectroscopy (CHERS) data analysis, and Dr. M. Bell for magnetics data analysis.

The authors are grateful to TFTR operation's research, engineering, and technical crews, and data acquisition and computing groups.

This work was supported by U.S. D.o.E. contract DE-AC02-76-CH03073. 


\section{Appendix A. FIELD PATTERN IN VACUUM TOROIDAL GE- OMETRY}

\section{Appendix A.1. Modifications to Simple Picture}

We examine field patterns in a realistic model of the TFTR tokamak. It has a toroidal vacuum vessel of a circular poloidal cross section and made of perfectly conducting walls. It also has a plasma of a circular cross section, but with its center in general not concentric with the vessel. Singular surfaces have a circular cross section, but with a center shifted with respect to the plasma geometrical center by a varying degree.

The following are improvements this more realistic representation can bring to our simplistic picture.

1. 'Cylindrical' Effects.

(a) Proximity effect.

(b) Multiple harmonics effect.

(c) Wall effect.

2. Toroidal Effects.

(a) Aspect ratio effect.

(b) Shafranov shift effects.

(c) 'Ballooning' effect.

We used the term, 'cylindrical' effects, simply as a contrast to the term, 'toroidal' effects. The former means those features that exist even in the absence of a toroidal geometry. We will explain briefly in turn what we mean by these effects.

The proximity effect refers to a varying distance between perturbation sensors and sources. Mirnov coils lie on an installation circle, which is in general not concentric with a singular surface where an MHD mode originates. The distance between an individual Mirnov coil and a singular surface thus varies around the poloidal circumference. Sensors closer to the perturbation source register a stronger signal than those further away. A multipole field decays strongly with the distance from its source $\left(\sim r^{-(m+1)}\right)$. The proximity effect can thus be the greatest source of a poloidal variation in an observed MHD mode pattern.

The multiple harmonics effect refers to altering a poloidal variation pattern by superposition of more than one poloidal harmonic. It can generate a poloidal standing wave pattern with nodal points where the modulus falls to zero, or becomes very small, and the argument experiences a jump, or varies rapidly. The following formula for superposition of two poloidal harmonics of the same amplitude but with 
m-numbers, $m_{1}$ and $m_{2}$, and relative phases, $\alpha_{1}$ and $\alpha_{2}$, illustrates the point:

$$
\begin{aligned}
& \cos \left(n \phi-m_{1} \theta-\omega t+\alpha_{1}\right)+\cos \left(n \phi-m_{2} \theta-\omega t+\alpha_{2}\right)= \\
& 2 \cos \left(\frac{\left(m_{1}-m_{2}\right)}{2} \theta-\frac{\left(\alpha_{1}-\alpha_{2}\right)}{2}\right) \cos \left(n \phi-\frac{\left(m_{1}+m_{2}\right)}{2} \theta-\omega t+\frac{\left(\alpha_{1}+\alpha_{2}\right)}{2}\right) .
\end{aligned}
$$

The first cosine factor on the right-hand side of the expression has neither the toroidal angle nor the time in its argument, and defines an 'amplitude' of oscillations that varies with the poloidal angle. The 'amplitude' vanishes at values of $\theta$ satisfying $\left(m_{1}-m_{2}\right) \theta / 2-\left(\alpha_{1}-\alpha_{2}\right) / 2= \pm \pi / 2$. These are nodal points of a standing wave. The argument takes a jump by $\pi$ at each node. Constituent harmonics with different amplitudes produce partial nodes and a less than $\pi$ jump at each of them.

The wall effect refers to the reaction of conducting walls to the presence of time varying magnetic perturbations. A perfectly conducting wall boundary condition requires that the field be tangent to the wall surface. Walls react to the presence of 'non-conforming' perturbations by generating current in its skin layer, which in turn produces a magnetic field that make the total perturbations 'conforming.'

The aspect ratio effect is the most basic consequence of a toroidal geometry. The field energy density becomes diluted on the outboard side by a volume element larger there than on the inboard side of a torus. Here, a finite aspect ratio refers to a Mirnov coil installation circle where perturbations are observed. But since the circle is an imaginary entity close to the vacuum vessel, the latter's geometry will serve as a surrogate in this section.

The aspect ratio effect also produces the 'Merezhkin effect:' the phase of perturbation field varies as function of the (geometrical) poloidal angle less rapidly on the outboard side than on the inboard side, reflecting the structure of confining field lines. The perturbation phase varies approximately linearly with the 'Merezhkin corrected angle' given by,

$$
\theta^{*} \approx \theta+\lambda_{M z k} \sin \theta
$$

where $\lambda_{M z k}$ is the 'Merezhkin coefficient.' We can obtain a value of the coefficient by fitting a curve to experimental argument data using the Merezhkin corrected angle. It has been found[50] that a value obtained from fitting often has a relationship to plasma properties,

$$
\lambda_{M z k} \approx \varepsilon_{p}\left(1+l_{i} / 2+\beta_{p}\right),
$$

where $\varepsilon_{p}, l_{i}$, and $\beta_{p}$ are the inverse aspect ratio of a singular surface, internal inductance, and poloidal $\beta$ of the plasma, respectively. We can therefore calculate a value of the Merezhkin coefficient from plasma properties.

The Shafranov shift has two consequences in the present context. A direct geometrical effect on the poloidal modulus variation is shifting of the center of a singular surface. This modifies the proximity effect by an amount dependent on the ratio of the singular surface to Mirnov circle radii. An indirect effect on the argument variation is intensifying of the Merezhkin effect. 
The ballooning effect refers to toroidal coupling of more than one MHD mode. It has a tendency to strengthen the perturbation intensity on the outboard side. If poloidal harmonics considered in Eq. A1 are MHD modes (assumed to belong to a single toroidal eigenmode), their relative phase disposition cannot be arbitrary, but is rather dictated by the ballooning condition: helices representing perturbations of the two modes would 'nestle' with each other in an energetically favorable orientation. More concretely speaking, if a poloidal harmonic has its maximum amplitude in the outboard midplane (where the magnetic energy density is at its minimum) at a certain toroidal angle, all other poloidal harmonics must also have their maximum at the same toroidal angle. Taking the ballooning effect into consideration in the present context would amount to selecting proper values for relative phases when dealing with multiple harmonics. Two harmonics with a unity difference in their poloidal harmonic numbers must have a relative phase of $\pi$.

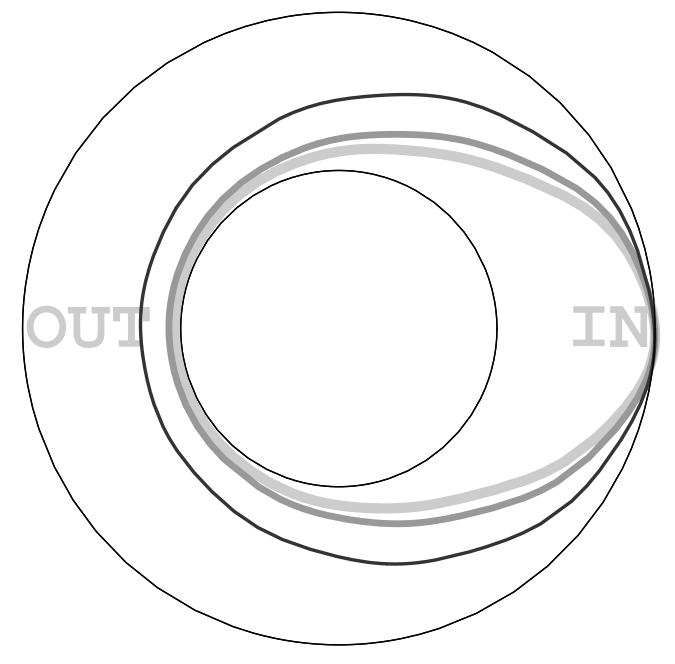

Fig. 40 Shows a basic inboard bias of a poloidal variation of the FFT modulus of Mirnov signals simulated by VACUUM Code. Perturbations originate on the plasma surface and are measured on the vacuum vessel wall. Curves, distinguished by a progressively darker shade of gray as well as a progressively narrower line width, are for small, medium, and large bore plasmas.

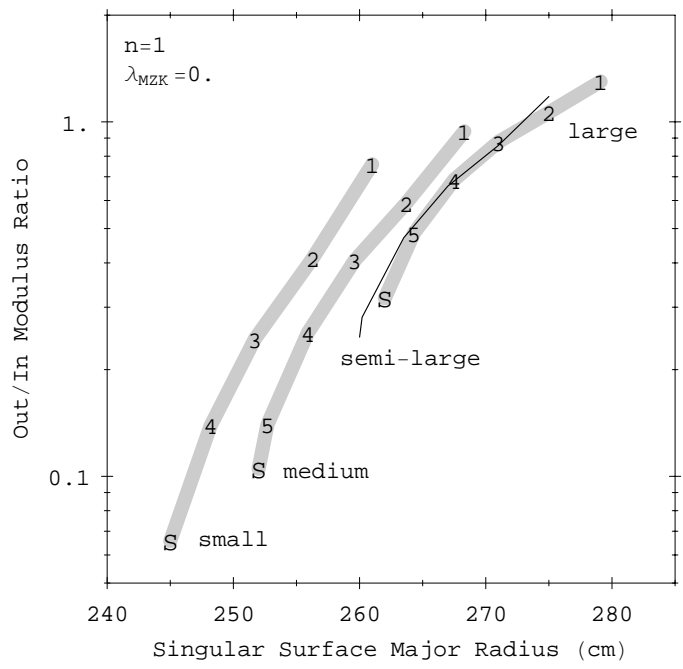

Fig. 41 Shows ranges of the in/out asymmetry ratio for small, medium, semi-large, and large bore plasmas calculated by VACUUM Code. Numerals, 1-5, indicate integer q-value surfaces on which perturbations originate. A letter, 'S,' means perturbations on the plasma surface.

\section{Appendix A.2. VACUUM Code Analysis}

We will use the VACUUM code[46] for investigating these geometrical effects. It assumes in the present calculations a form of perturbations (e.g., $\mathrm{m} / \mathrm{n}=5 / 1$ ) on a source surface, which is a toroidal surface of a circular cross section. The perturbation intensity is constant on the source surface. The code solves the Maxwell's equations in the assumed vacuum space defined by the source surface and vacuum vessel walls. The solution satisfies a conducting wall boundary condition on the vessel walls, and matches prescribed perturbations on the source surface. The 
VACUUM code is a powerful tool for investigating a magnetic field pattern in a general geometry in a computationally rigorous manner.

The VACUUM code produces a complex valued tangential magnetic field at the vessel walls for a set of poloidal angles at a single toroidal angle. A complex valued toroidal variation, $\exp i n \phi(t)$, is introduced to the complex valued field as a function of time to generate a time series signal at each 'coil' location. We will use sixty four such coils in the following analysis. The FFT modulus and argument of a simulated signal are treated in the same manner as those of an experimental signal.

Basic Inboard Bias - Two geometrical characteristics of TFTR produce a basic bias in the poloidal modulus variation in favor of the inboard side. First, the aspect ratio effect favors the inboard side. VACUUM code calculations show that the in/out asymmetry ratio is about two for a singular surface that is concentric with the vessel (and hence has an aspect ratio effect but no direct proximity effect). Second, an operational proximity effect exists. Recall that the last closed flux surface (plasma boundary) is in contact with limiters on the inboard side under usual operating conditions. The plasma geometrical center is thus on the inboard side of the vessel center by up to several $\mathrm{cm}$ for a large bore plasma and twenty $\mathrm{cm}$ for a small bore plasma. A singular surface inside the plasma also has a tendency to be geometrically closer to Mirnov coils on the inboard side than on the outboard side. Two effects to be described below, the Shafranov shift effect and multiple harmonics superposition, will counter the basic inboard bias.

Figure 40 demonstrates the basic inboard bias. It shows the poloidal variations of the FFT modulus of Mirnov signals simulated by the VACUUM code for small, medium, and large bore plasmas. The calculations here assumed that $\mathrm{m} / \mathrm{n}=5 / 1$ perturbations originate on the plasma surface. Rapid decay of a multipole field produces large differences between the inboard and outboard sides for all cases. The difference is particularly extreme for a small bore plasma.

The basic inboard bias appears in a more quantitative manner in Fig. 41. It includes many features for future purposes as well as the bias effect presently under discussion. But we will explain the figure's construction here. It shows the out/in asymmetry ratio for a number of singular surfaces with an integer q-value (here $\mathrm{n}=1$ ) in small, medium, and large bore plasmas. The calculations needed major and minor radii of singular surfaces, which depend on a q-profile. Discharges, \#66869 (small bore), \#73440 (medium), and \#81178 (large), served here as a representative of their respective classes. An asymmetry ratio for a singular surface appears as a numeral corresponding to its integer q-value, and is plotted against its major radius. The ratio designated by ' $\mathrm{S}$ ' means that perturbations lie on the plasma surface, and have an m-number corresponding to the first integer q-value surface outside the plasma boundary. The in/out asymmetry ratio (reciprocal of values shown in the figure) is 15.2, 9.6, and 3.1 for the small, medium, and large bore plasmas, respectively, when perturbations lie on the plasma surface. We will return to this figure repeatedly in the course of discussion.

Shafranov Shift Effect - For an interior singular surface the Shafranov shift 
counters a basic inboard bias as illustrated in Fig. 42. A q-profile and shifted circular flux surfaces, computed by the SNAP code for an actual 'semi-large' bore discharge, is a basis for these VACUUM code calculations. The shot \#91561 used here is slightly smaller than a standard large bore plasma - see Table 2.4. Figure 2 shows these flux surfaces and their centers. Perturbations originate on an integer $\mathrm{q}$-value singular surface with a corresponding m-number and $n=1$. The Shafranov shift moves the center of a singular surface farther outboard for a low m-number perturbations. The surface also becomes farther away from the sensor circle. Both contribute to restoring the symmetry.

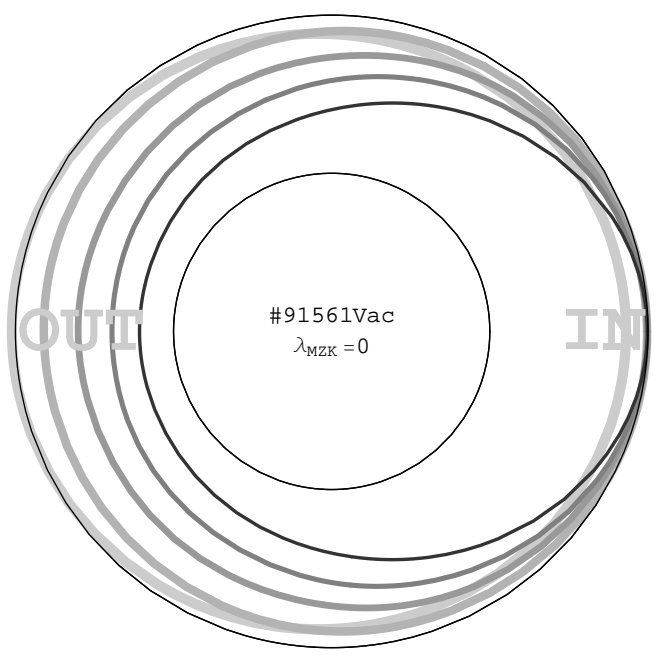

Fig. 42 Shows Shafranov shift effect on a poloidal variation of the FFT modulus of Mirnov signals simulated by VACUUM Code for a semi-large bore plasma. Perturbations $(n=1)$ originate on integer- $q$ singular surfaces computed for an actual discharge. The surfaces have a different amount of Shafranov shift. Curves, distinguished by a progressively darker shade of gray as well as a progressively narrower width, are for $\mathrm{m}=1$ through $\mathrm{m}=5$.

Return to Fig. 41 for a more quantitative representation of the Shafranov shift effect. Important to note is that the out/in asymmetry ratio for a $\mathrm{q}=1$ singular surface (smallest m-number perturbations) is somewhat below (0.76), just below (0.94), and somewhat above (1.30), unity for small, medium, and large bore plasmas. In other words, even perturbations on an inner-most integer q-value surface cannot produce a strongly outboard leaning poloidal variation. The corresponding numbers for a $\mathrm{q}=2$ surface are $0.41,0.59$, and 1.06 for small, medium, and large bore plasmas, respectively. The smaller plasmas will have a strongly inboard biased variation, and a large bore plasma can produce an essentially centered poloidal variation, on a $\mathrm{q}=2$ surface. The out/in asymmetry ratio for a semi-large bore plasma appears in the figure as a thin black curve, though without numerals identifying calculated points, in order to show its relationship with the other size plasmas.

Vertical Offset Effect - Figure 43 shows the effect on the modulus variation of vertically offsetting the plasma center. Perturbations, $\mathrm{m} / \mathrm{n}=5 / 1$, are on a $\mathrm{q}=5$ surface calculated by the SNAP code for an actual semi-large bore plasma shot, \#91561. A combination of a vertical offset and a basic inboard bias produced a 'lobe-like' feature that extends in a lower inboard direction. The figure shows that generating a modest (visually) up/down asymmetry requires a large vertical offset.

A more quantitative assessment of the vertical offset effect is in Fig. 44. It shows the down/up asymmetry ratio for small, medium, and large bore plasmas as 
a function of the vertical offset distance. But the result is nearly the same for all three cases. These are model plasmas with major/minor radii of $245 / 80,252 / 87$, and $262 / 97 \mathrm{~cm}$ on the midplane. The vertical position then moves to $-2,-5$, and $-10 \mathrm{~cm}$, while keeping the major radius unchanged. Perturbations, $\mathrm{m} / \mathrm{n}=5 / 1$, are on a surface with a minor radius equaling $90 \%$ of the plasma minor radius. 'Dots' are calculated results, and curves are interpolations.

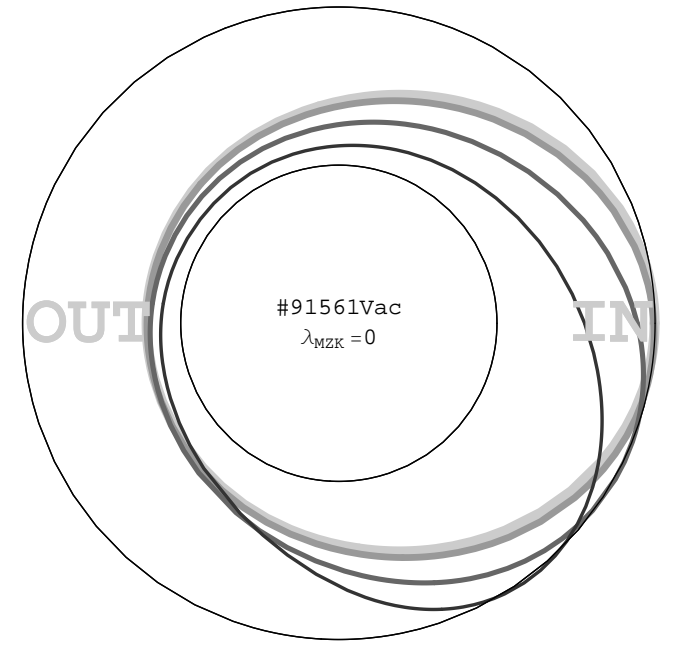

Fig. 43 Shows effect of vertical offset of singular surface location on a poloidal variation of the FFT modulus of Mirnov signals simulated by VACUUM Code. Curves, distinguished by a progressively darker shade of gray as well as a progressively narrower line width, are for an offset of $0,-1,-5$, and $-10 \mathrm{~cm}$.

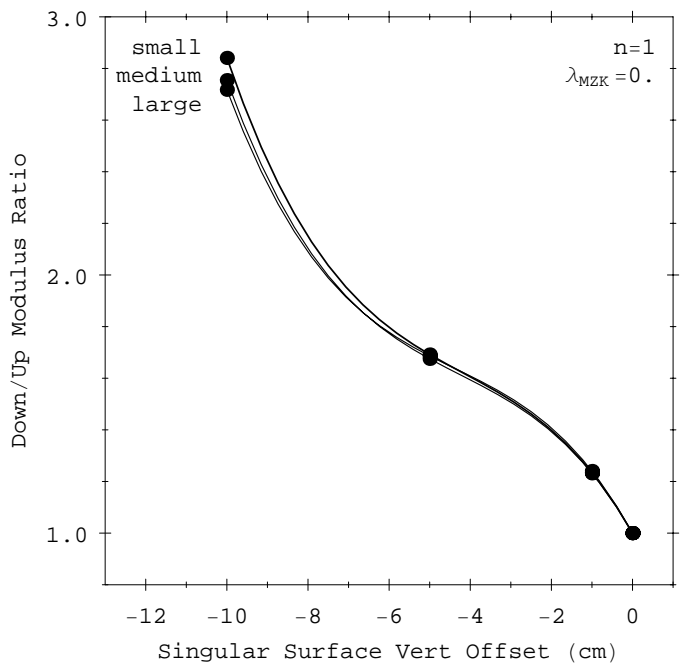

Fig. 44 Summarizes vertical offset effect (previous figure) in terms of the down/up asymmetry ratio plotted against the shift of singular surface location.

A model plasma comes in contact with bumper limiters at an off-midplane point, and its minor radius changes, as it is offset vertically. At an extreme position shown in the figure, $-10 \mathrm{~cm}$, the plasma minor radius and contact angle (in parentheses) are $77.6 \mathrm{~cm}(-27 \mathrm{deg}), 83.6 \mathrm{~cm} \mathrm{(-38} \mathrm{deg),} \mathrm{and} 89.6 \mathrm{~cm} \mathrm{(-73} \mathrm{deg)} \mathrm{for} \mathrm{the} \mathrm{small,} \mathrm{medium,}$ and large bore plasmas, respectively. At this offset an actual large bore plasma would have contacted a point on the RF limiters in the TFTR (see Fig. 2). A new contact point implies interaction of the plasma with an area of limiters that is not routinely conditioned. The effect of both minor radius and contact angle changes would be so traumatic operationally on the plasma performance that an unintentional vertical offset of this magnitude is impossible to go unnoticed. Tokamak operators would in fact detect a far smaller offset in practice.

Multiple Harmonics Effect - Figure 45 is a modulus variation when two MHD modes, $\mathrm{m} / \mathrm{n}=4 / 1$ and $5 / 1$, shown in Fig. 42 are superposed with the same amplitude but with a $\pi$ phase difference. Reinforcement on the outboard side and cancellation on the inboard side between the two modes' signal intensities overcame a basic inboard bias. This becomes evident by setting $\left(\alpha_{1}-\alpha_{2}\right)=\pi$ and $\left(m_{1}-m_{2}\right)=1$ in Eq. A1, as $\theta=\pi$ (outboard midplane) and $\theta=0$ (inboard midplane) yield 
maximum and minimum amplitudes. The superposition produced a moderately outboard leaning variation with an out/in asymmetry ratio of 1.3. Note the out/in and up/down asymmetries written on a lower left corner of this modulus variation figure and others to follow.

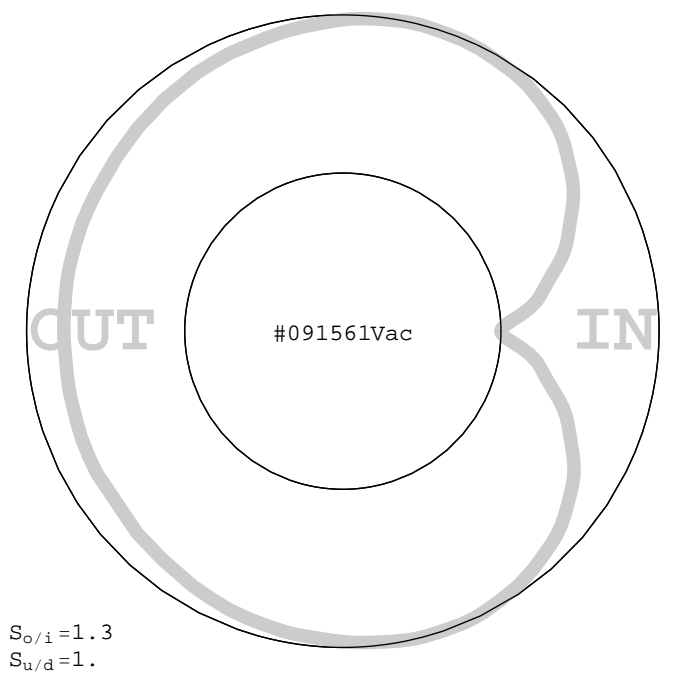

Fig. 45 Shows effect of superposing two harmonics of the same amplitude on a poloidal variation of the FFT modulus of Mirnov signals simulated by VACUUM Code. Harmonics are $\mathrm{m} / \mathrm{n}=4 / 1$ and $5 / 1$.

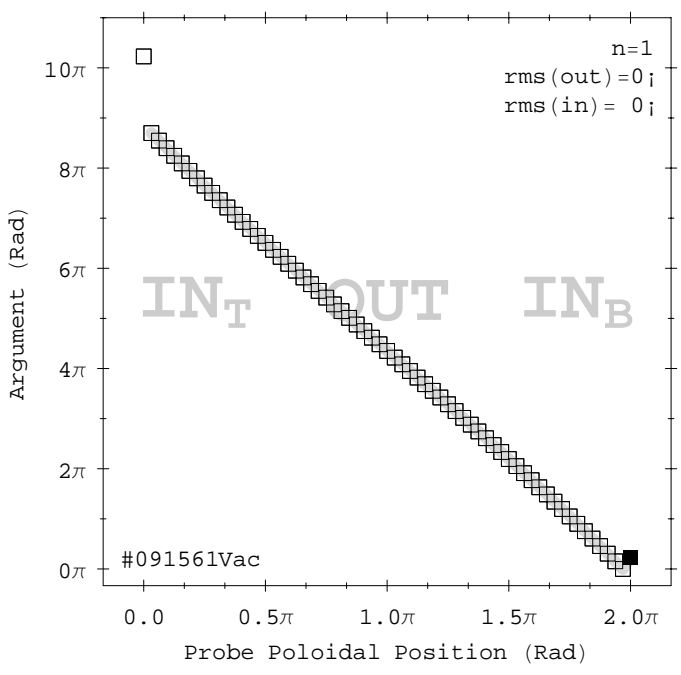

Fig. 46 Shows effect of superposing two harmonics of the same amplitude on a poloidal variation of the FFT argument of Mirnov signals simulated by VACUUM Code. Harmonics are $\mathrm{m} / \mathrm{n}=4 / 1$ and $5 / 1$.

A nodal point occurs at the inboard midplane when two modes have the same amplitude as in the present case. This happens even though the poloidal angle for the modes are measured about different Shafranov shifted centers, because the position designated by 0 or $\pi$ is independent of the locations of these centers. The unity difference in the m-numbers is clearly a pre-requisite for this kind of multiple harmonic effect to occur. An argument variation of the superposed modes is in Fig. 46. A jump in the argument is produced at the node. The overall slope of the curve is about $\left(m_{1}-m_{2}\right) / 2=4.5$. In order to overcome a basic inboard bias by a significant margin we must thus have superposition of two modes with a unity m-number difference and comparable amplitudes.

A modulus variation of another set of superposed modes is in Fig. 47. The modes are $\mathrm{m} / \mathrm{n}=2 / 1$ and $5 / 1$, and have the same amplitude. Their Shafranov shifted singular surfaces come from a q-profile computed by the SNAP code for an actual large bore discharge, \#81178. The modulus variation has three nodal points, one at the inboard midplane and two at approximately 120 deg away on the top and bottom sides. But different Shafranov shifts caused poloidal variations to be measured about different centers, and made the nodal points partial, except the one at the inboard midplane. Unequal mode amplitudes would make a midplane node also partial. An m-number difference other than unity could not generate an outboard lean large enough to overcome a basic inboard bias. The resultant 
modulus variation is still inboard leaning with an out/in asymmetry ratio of 0.89 . An argument variation is in Fig. 48. 'Jumps' at nodal points are modest except at the midplane.

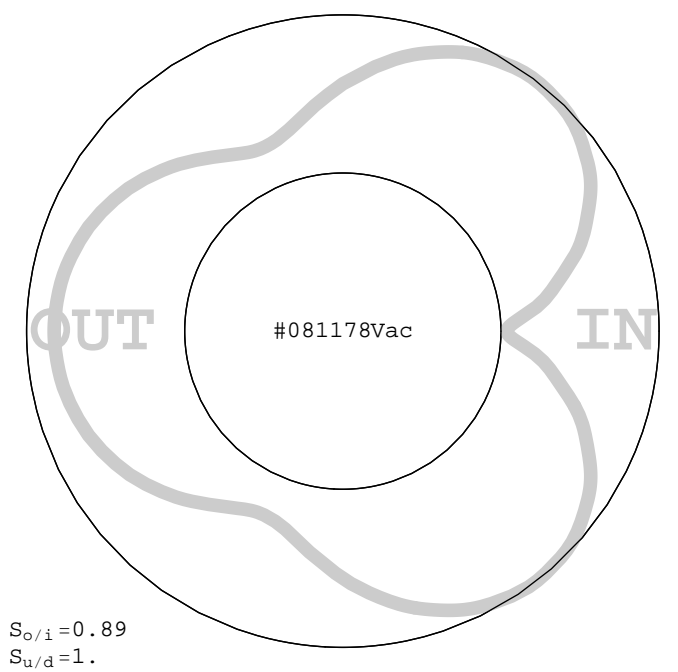

Fig. 47 Shows effect of superposing two harmonics of the same amplitude on a poloidal variation of the FFT modulus of Mirnov signals simulated by VACUUM Code. Harmonics are $\mathrm{m} / \mathrm{n}=2 / 1$ and $5 / 1$.

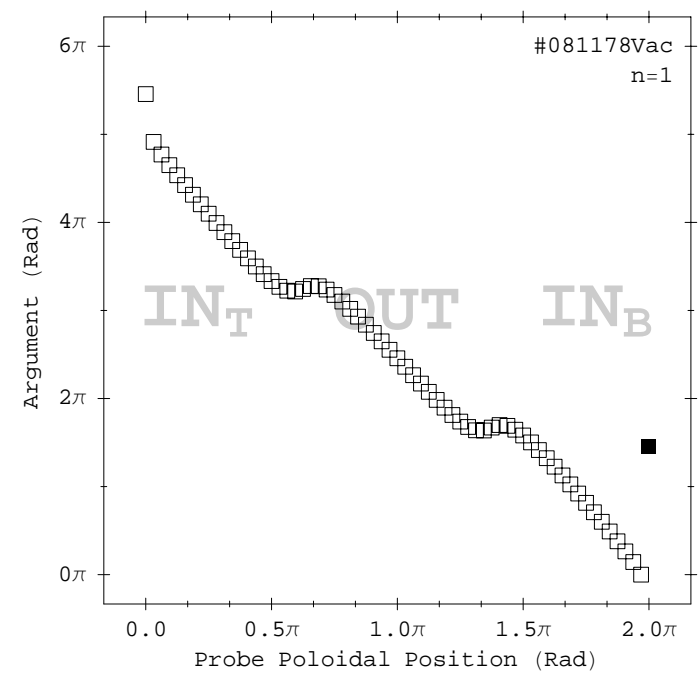

Fig. 48 Shows effect of superposing two harmonics of the same amplitude on a poloidal variation of the FFT argument of Mirnov signals simulated by VACUUM Code. Harmonics are $\mathrm{m} / \mathrm{n}=2 / 1$ and $5 / 1$.

Merezhkin Effect - Two curves in Fig. 49 illustrate influence of the Merezhkin effect on the poloidal modulus variation. Perturbations, $m / n=5 / 1$, are on a $q=5$ surface calculated by the SNAP code for an actual semi-large bore shot, \#91561. They have a sinusoidal poloidal variation on the source surface, but with respect to the Merezhkin-corrected angle rather than a geometrical angle in the physical space. The two curves are for coefficient values of 0.6 and 0.8 . The influence is barely noticeable for a coefficient value below 0.5. A 'flat face' appears on the inboard side for values around 0.6. A 'dimple' develops for values about 0.7, and becomes quite pronounced for values around 0.8 and greater.

A likely origin of Merezhkin dimple is 'bunching' of a multipole structure on the inboard side. The field line pitch angle is 'shallow' on the inboard side and 'steep' on the outboard side. (A field line travels a smaller angle in a poloidal direction for a unit angle in a toroidal direction on the inboard side than on the outboard side.) A multipole structure representing the field line on the inboard side is effectively of a higher order than a nominal value. The field decays more rapidly on the inboard side, and creates a dimple.

The VACUUM code calculations discussed above used a q-profile from a specific shot in each bore size of the plasma. The result would be applicable to other discharges to the extent that their q-profiles are not too different from that of the representative discharges. With respect to $n=1$ MHD modes in TFTR, the VACUUM code result says the following: 
1. A usually outermost mode, $\mathrm{m}=5$, has a strong inboard bias. The in/out asymmetry ratio is about 15,7 , and 2 for small, medium, and large bore discharges.

2. No mode can generate an out/in asymmetry greater than about 1.3. A maximum value is achieved by an $\mathrm{m}=1$ mode in a large bore discharge.

3. No single off-axis $(m>1)$ mode can produce an outboard lean of significance. The maximum out/in asymmetry ratio is about unity produced by an $m=2$ mode in a large bore discharge.

4. Only a superposition of two modes with a unity m-number difference can create a outboard leaning variation of significance.

5. An down/up asymmetry ratio of 3 means a vertical offset in excess of $-10 \mathrm{~cm}$ in any size plasma.

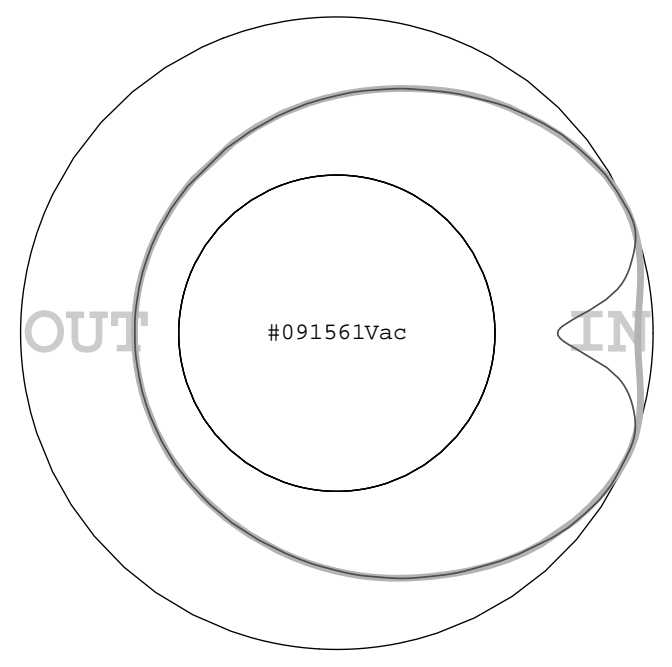

Fig. 49 Shows Merezhkin effect on a poloidal variation of the FFT modulus of simulated Mirnov signals. Perturbations $(\mathrm{m} / \mathrm{n}=5 / 1)$ originate on a $\mathrm{q}=5$ singular surface computed for an actual discharge. Curves, distinguished by the shade of gray as well as the line width, are for a Merezhkin coefficient of 0.6 and 0.8 .

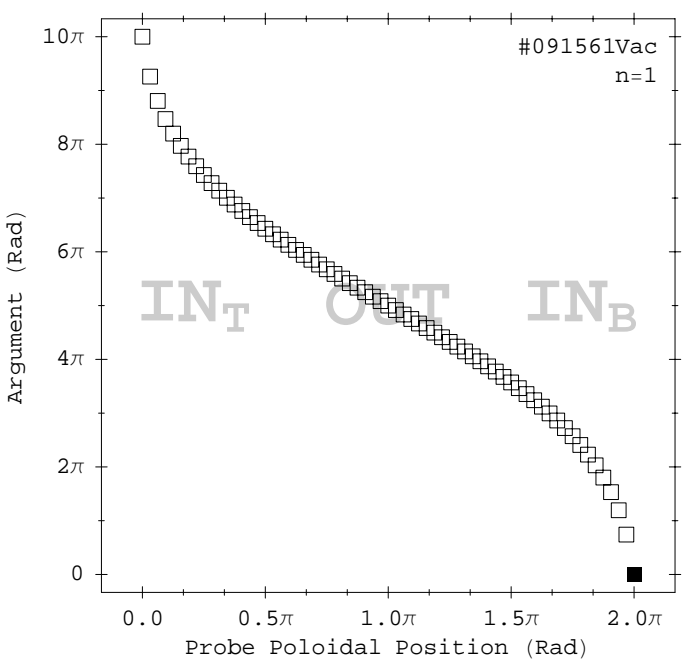

Fig. 50 Shows Merezhkin effect on a poloidal variation of the FFT argument of simulated Mirnov signals. Merezhkin coefficient is 0.8. See the previous figure.

\section{Appendix B. GEOMETRICAL CORRECTION}

We consider the variation of a multipole perturbation field in two-dimensional vacuum space. The perturbation field originates on a 'perturbation circle.' Mirnov coils measure the perturbation field on a 'Mirnov circle' that is in general not concentric with the perturbation circle. We want to calculate approximately a perturbation filed on a circular 'virtual wall' that is concentric with the perturbation circle. We derive formulas for 'geometrical corrections.' One type of corrections are for the distance and another is for the angle. The latter corrections are minor, and usually 
ignorable. Multiplying Mirnov signals by both corrections will yield a perturbation field 'measured' on the virtual wall.

Figure 51 explains the geometry. A Mirnov circle of radius, $a_{M C}$, is centered at $O_{M C}\left(x_{M C}, y_{M C}\right)$ in a $(\mathrm{x}, \mathrm{y})$ coordinate system. A perturbation circle of radius, $a_{m n}$, is centered at $O_{m n}\left(x_{m n}, y_{m n}\right)$. A virtual wall circle of radius, $a_{v w}$, is centered also at $O_{m n}\left(x_{m n}, y_{m n}\right)$. Both $a_{M C}$ and $a_{v w}$ are assumed to be greater than $a_{m n}$. Consider a point on the Mirnov circle. A vector drawn from the center of the perturbation circle to the point intersects the perturbation circle, virtual wall, and Mirnov circle at points, 'P,' 'V,' and 'M,' respectively. The distance from the center to these three points are $a_{m n}, a_{v w}$, and $s_{m n}$, respectively. Angles that the vector makes about the two centers are $\theta_{M C}$ and $\theta_{m n}$.

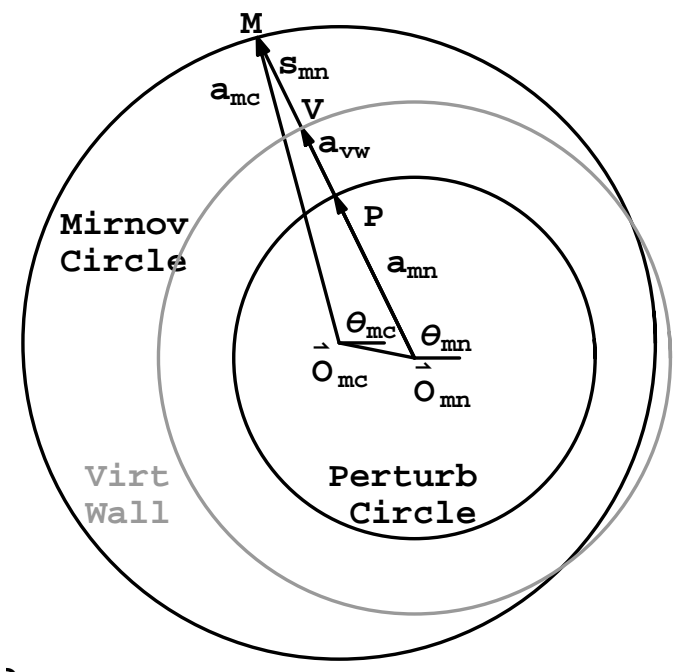

Fig. 51 Geometry of Mirnov, perturbation, and virtual wall circles.

The angle measured about the perturbation center is given in terms of the angle about the Mirnov center by

$$
\theta_{m n}\left(\theta_{M C}\right)=\arctan a_{M C} \sin \theta_{M C}+\frac{\left(y_{M C}-y_{m n}\right)}{a_{M C} \cos \theta_{M C}+\left(x_{M C}-x_{m n}\right)} .
$$

The (square of) length of the vector to the point on the Mirnov circle is given in terms of the angle about the Mirnov center by,

$$
\left(s_{m n}\left(\theta_{M C}\right)\right)^{2}=\left(a_{M C} \cos \theta_{M C}+\left(x_{M C}-x_{m n}\right)\right)^{2}+\left(a_{M C} \sin \theta_{M C}+\left(y_{M C}-y_{m n}(\mathrm{~B}) 2 .\right)\right.
$$

We assume that the multipole field outside the perturbation circle varies as an inverse $(\mathrm{m}+1)$ power of the distance measured from the center of the circle. The distance geometrical corrections are given by,

$$
g_{d}\left(\theta_{M C} ; m\right)=\left(s_{m n}\left(\theta_{M C}\right) / a_{v w}\right)^{(m+1)} .
$$

Mirnov coils are usually installed with their axes tangent to the Mirnov circle. When the Mirnov circle is not concentric with the perturbation circle, they will measure a component of the perturbation filed that is not perfectly tangent to the perturbation circle. The angle geometrical corrections are given by,

$$
g_{a}\left(\theta_{M C} ; m\right)=1 / \cos \left(\theta_{M C}-\theta_{m n}\right) .
$$




\section{References}

[1] C. Kessel, J. Manickam, G. Rewoldt, and W. M. Tang, Phys. Rev. Lett. 72, $1212(1994)$.

[2] F.M. Levinton and M.C. Zarnstorff, Phys. Rev. Lett. 75(1995)4417.

[3] E.J. Strait, et al., Phys. Rev. Lett. 75(1995)4421.

[4] Y. Kamada, et al., Nucl. Fusion 39(1999)1845.

[5] B.W. Rice, et al., Nucl. Fusion 39(1999)1855.

[6] JET Team, Nucl. Fusion 39(1999)1875.

[7] V.S. Chan, C.M. Greenfield, L.L. Lao, T.C. Luce, C.C. Petty, G.M. Staebler and the DIII-D Team, Nucl. Fusion 40(2000)1137.

[8] M.F.F. Nave and J.A. Wesson, in Controlled Fusion and Plasma Physics, 1987, Proc. of 14h European Conference, Madrid (EPS, Geneva, 1987), Vol. 3, p. 1103.

[9] J.A. Snipes, D.J. Campbell, P.S. Hayes, T.C. Hender, M. Hugon, P.J. Lomas, N.J. Lopes Cardozo, M.F.F. Nave, and F.C. Schüller, "Large Amplitude QuasiStationary MHD Modes in JET," Nucl. Fusion, 28(1988)1085.

[10] R. Fitzpatrick and T.C. Hender, Phys. Fluids B 3(1991)644.

[11] J.T. Scoville, R.J. La Haye, A.G. Kellman, T.H. Osborne, R.D. Stambaugh, E.J. Strait, and T.S. Taylor, Nucl. Fusion, 31(1991)875.

[12] R.J. La Haye, Nucl. Fusion, 31(1991)1550.

[13] R.J. La Haye, R. Fitzpatrick, T.C. Hender, A.W. Morris, J.T. Scoville, and T.N. Todd, Phy. Fluids B 4(1992)2098.

[14] T.C. Hender, R. Fitzpatrick, A.W. Morris, P.G. Carolan, R.D. Durst, T. Edlington, J. Ferreira, S.J. Fielding, P.S. Haynes, J. Hugill, I.J. Jenkins, R.J. La Haye, B.J. Parham, D.C. Robinson, T.N. Todd, M. Valovič, G. Vayakis, Nucl. Fusion, 32(1992)2091.

[15] W. Morris, P.G. Carolan, R. Fitzpatrick, T.C. Hender, and T.N. Todd, Phys. Fluids, B 4(1992)413

[16] G.M. Fishpool and P.S. Haynes, Nucl. Fusion, 34(1994)109.

[17] H. Takahashi, Fusion Plasma Diagnostics, 7th Int. Toki Conf. on Plasma Physics and Controlled Nuclear Fusion, Toki, Japan, 1995 (Fusion Engineering and Design, 34-35(1997)89).

[18] R.J. Buttery, et al., Nucl. Fusion 39(1999)1827. 
[19] R. Carrera, R.D. Hazeltine, and M. Kotschenreuther, Phys. Fluids, 29(1986)899.

[20] Z. Chang, J.D. Callen, E.D. Fredrickson, R.V. Budny, C.C. Hegna, K.M. McGuire, M.C. Zarnstorff, and TFTR group, Phys. Rev. Lett. 74(1995)4663.

[21] R.J. La Haye, et al., in Fusion Energy 1996, Proc. of the 16th IAEA Conference, Montreal, 1996 (Int. Atomic Energy Agency, Vienna, 1997), Vol. 1, p. 747.

[22] D.A. Gates, B. Lloyd, A.W. Morris, G. McArdle, M. O’Brien, M. Valovic, C.D. Warrick, H.R. Wilson, and The COMPASS-D and ECRH Teams, in Fusion Energy 1996, Proc. of the 16th IAEA Conference, Montreal, 1996 (Int. Atomic Energy Agency, Vienna, 1997), Vol. 1, p. 715.

[23] C. Ren, J.D. Callen, T.A. Gianakon, C.C. Hegna, Z. Chang, E.D. Fredrickson, K.M. McGuire, G. Taylor, M.C. Zarnstorff, Physics of Plasmas, 5(1998)450.

[24] Z. Chang, E.D. Fredrickson, S.H. Batha, M.G. Bell, R.V. Budny, F.M. Levington, K.M. McGuire, G. Taylor, M.C. Zarnstorff, and the TFTR group, Physics of Plasmas, 5(1998)1076.

[25] A. Bondeson and D.J. Ward, Phys. Rev. Lett. 72(1994)2709.

[26] R. Betti and J.P. Freidberg, Phys. Rev. Lett. 74(1995)2949.

[27] A. H. Boozer, Phys. Plasmas 2(1995)4521.

[28] M.S. Chu, et al., Phys. Plasmas 2(1995)2236.

[29] E.J. Strait, et al., Phys. Rev. Lett. 74(1995)2483.

[30] A.D. Turnbull, et al, in Proc. of the 15th Int. Conf, Seville, 1994 (Plasma Phys. Controlled Nucl. Fusion Res. 1(1995)705.

[31] T.S. Taylor, et al, Phys. Plasmas 2(1995)2390.

[32] R. Betti, Phys. Plasmas 5(1998)3615.

[33] M. Okabayashi, et al, Nucl. Fusion 36(1996)1167.

[34] T.H. Ivers, et al, Phys. Plasmas 3(1996)1746.

[35] Garofalo, A.M., Turnbull, A.D., Austin, M.E., Bialek, J., Chu, M.S., Comer, K.J., Fredrickson, E.D., Groebner, R.J., La Haye, R.J., Lao, L.L., Lazarus, E.A., Navratil, G.A., Osborne, T.H., Rice, B.W., Sabbagh, S.A., Scoville, J.T., Strait, E.J., and Taylor, T.S., Phys. Rev. Lett., 82(1999)3811.

[36] E.J. Strait, et al., Nucl. Fusion 39(1999)1977.

[37] F. Wagner, Phys. Rev. Lett. 49(1982)1408.

[38] Schaffer, M.J. and Leikind, B.J., Nucl. Fusion 31(1991)1750. 
[39] H. Zohm, Plas. Phys. Contr. Fusion 38, 105 (1996).

[40] R.J. Hawryluk, et al., Phys. Plasmas 5(1998)1577.

[41] E.D. Fredrickson, K. McGuire, A. Cavallo, B. Grek, K.-I. Hattori, D. Johnson, and A.W. Morris, Rev. Sci. Instrum. 59(1988)1797.

[42] A. Cavallo, R.C. Cutler, and M.P. McCarthy, Rev. Sci. Instrum. 59(1988)889.

[43] G. Taylor, C.E. Bush, E.D. Fredrickson, H.K. Park, and A.T. Ramsey, Nucl. Fusion 32(1992)1867.

[44] R.V. Budny, Nucl. Fusion 34(1994)1247, and references therein.

[45] H.H. Towner, R.J. Goldston, G.W. Hammett, J.A. Murphy, C.K. Phillips, S.D. Scott, M.C. Zarnstorff, D. Smithe, Rev. Sci. Instrum. v. 63(1992)4753.

[46] Chance, M., Physics Plasmas, 4(1977)2161.

[47] E.D. Fredrickson, private communication, Princeton Plasma Physics Lab., 2000 .

[48] R. B. White, D. A. Monticello, M. N. Rosenbluth and B. V. Waddell, Phys. Fluids, 20(1977)800, Eq. 9.

[49] R.C. Grimm, J.M. Greene, and J.L. Johnson, p.p. 253-280, Vol. 16, Methods in Computational Physics, J. Killeen, ed., Academic Press, New York, 1976.

[50] Merezhkin, V.G., Soviet J. Plasma Physics, 4(1978)152.

[51] G. Bateman, MHD Instabilities, The MIT Press, Cambridge, Mass., 1978.

[52] P.H. Rutherford, Phys. Fluids, 16(1973)1903.

[53] H.P. Furth, J. Killeen, and M.N. Rosenbluth, Phys. Fluids, 6(1963)459.

[54] H. Takahashi, E. Fredrickson, and K. McGuire, Bull. Am. Phys. Soc., 39 (1994) $1681-1682$.

[55] H. Takahashi, E. Fredrickson, and K. McGuire, Bull. Am. Phys. Soc., 40 (1995) 1870 .

[56] H. Takahashi, E. Fredrickson, and K. McGuire, Bull. Am. Phys. Soc., 41 (1996)1380.

[57] H. Takahashi, E. Fredrickson, K. McGuire, and A. Ramsey, Bull. Am. Phys. Soc., 42 (1997) 2040.

[58] H. Takahashi and E. Fredrickson, Bull. Am. Phys. Soc., 43 (1998) 1827.

[59] H. Takahashi, E.D. Fredrickson, M.J. Schaffer, T.E. Evans, and L.L. Lao, Bull. Am. Phys. Soc., 44 (1999) 76. 
[60] H. Takahashi, E.D. Fredrickson, M.J. Schaffer, T.E. Evans, L.L. Lao, and J.G. Watkins, Bull. Am. Phys. Soc., 45 (2000) 153.

[61] J.L. Luxon and L.G. Davis, Fusion Technol. 8(1985)441.

[62] See references in a review paper, McCracken, G.M., J. Nucl. Mat. $93 \& 94(1980) 3$.

[63] Goodall, D.H.J., J. Nucl. Mat. 93\&94(1980)154.

[64] Schaffer, M.J., Evans, T.E., Humphreys, D.A., Hyatt, A.W., Kellman, A.G., and LaHaye, R.J., Poster 3Q21, APS 1996, Denver, CO, Nov. 11-15.

[65] R.S. Granetz, I.H. Hutchinson, J. Sorci, J.H. Irby, B. LaBombard, and D. Gwinn, Nucl. Fusion, 36(1996)545.

[66] Evans, T.E., Kellman, A.G., Humphreys, D.A., Schaffer, M.J., Taylor, P.L., Whyte, D.G., Jernigan, T.C., Hyatt, A.W., and Lee, R.L., J. Nucl. Mat. 241243(1997)606.

[67] S. Wolfram, The Mathematica Book, 4th ed., Cambridge Univ. Press, 1999, p. 903.

[68] E. O. Brigham, The Fast Fourier Transform and Its Applications, Sec. 3.4, Prentice-Hall, Englewood Cliffs, New Jersey, 1988. 


\section{External Distribution}

Plasma Research Laboratory, Australian National University, Australia

Professor I.R. J ones, Flinders University, Australia

Professor J oão Canalle, Instituto de Fisica DEQ/IF - UERJ , Brazil

Mr. Gerson O. Ludwig, Instituto Nacional de Pesquisas, Brazil

Dr. P.H. Sakanaka, Instituto Fisica, Brazil

The Librarian, Culham Laboratory, England

Library, R61, Rutherford Appleton Laboratory, England

Mrs. S.A. Hutchinson, JET Library, England

Professor M.N. Bussac, Ecole Polytechnique, France

Librarian, Max-Planck-Institut für Plasmaphysik, Germany

J olan Moldvai, Reports Library, MTA KFKI-ATKI, Hungary

Dr. P. Kaw, Institute for Plasma Research, India

Ms. P.J . Pathak, Librarian, Insitute for Plasma Research, India

Ms. Clelia De Palo, Associazione EURATOM-ENEA, I taly

Dr. G. Grosso, Instituto di Fisica del Plasma, Italy

Librarian, Naka Fusion Research Establishment, J AERI, J apan

Library, Plasma Physics Laboratory, Kyoto University, J apan

Research Information Center, National Institute for Fusion Science, J apan

Dr. O. Mitarai, Kyushu Tokai University, J apan

Library, Academia Sinica, Institute of Plasma Physics, People's Republic of China

Shih-Tung Tsai, Institute of Physics, Chinese Academy of Sciences, People's Republic of China

Dr. S. Mirnov, TRINITI, Troitsk, Russian Federation, Russia

Dr. V.S. Strelkov, Kurchatov Institute, Russian Federation, Russia

Professor Peter Lukac, Katedra Fyziky Plazmy MFF UK, Mlynska dolina F-2, Komenskeho Univerzita, SK-842 15 Bratislava, Slovakia

Dr. G.S. Lee, Korea Basic Science Institute, South Korea

Mr. Dennis Bruggink, Fusion Library, University of Wisconsin, USA

Institute for Plasma Research, University of Maryland, USA

Librarian, Fusion Energy Division, Oak Ridge National Laboratory, USA

Librarian, Institute of Fusion Studies, University of Texas, USA

Librarian, Magnetic Fusion Program, Lawrence Livermore National Laboratory, USA

Library, General Atomics, USA

Plasma Physics Group, Fusion Energy Research Program, University of California at San Diego, USA

Plasma Physics Library, Columbia University, USA

Alkesh Punjabi, Center for Fusion Research and Training, Hampton University, USA

Dr. W.M. Stacey, Fusion Research Center, Georgia Institute of Technology, USA

Dr. J ohn Willis, U.S. Department of Energy, Office of Fusion Energy Sciences, USA

Mr. Paul H. Wright, Indianapolis, Indiana, USA 
The Princeton Plasma Physics Laboratory is operated by Princeton University under contract with the U.S. Department of Energy.

\author{
Information Services \\ Princeton Plasma Physics Laboratory \\ P.O. Box 451 \\ Princeton, NJ 08543
}

Phone: 609-243-2750

Fax: 609-243-2751

e-mail: pppl_info@pppl.gov

Internet Address: http://www.pppl.gov 